\title{
COMPUTING SPECTRAL FLOW VIA CUP PRODUCTS
}

\author{
PAUL KIRK \& ERIC KLASSEN
}

\section{Introduction}

In this paper we investigate the spectral flow of the "Dirac" operator $D_{A}=* d_{A}-d_{A} *$ acting on $0+2$ forms on a 3-manifold $M$ as $A$ varies in the connections in an $S U(2)$-bundle $P$ over $M$. This operator arises as the tangential operator (in the sense of [1]) for the signature and selfduality operators on a 4-manifold, and as a stabilization of the Hessian of the Chern-Simons function on the space of gauge equivalence classes of connections on $P$. In the case where $A$ is flat, it is a square root of the (twisted) Laplacian $\Delta_{A}$ acting on even forms.

Our main contribution to the calculation of the spectral flow of these operators is as follows. If $A_{t}$ is a path of flat connections on a manifold $M$ which either has boundary (Theorem 4.8) or is closed (Theorem 5.1), then we show that computation of the "local contribution to spectral flow at time $t=0$ " (i.e., the slopes of the eigenvalues that are crossing 0 at $t=0$ ) can be reduced to a cup product computation in the cohomology of $M$ with twisted coefficients. Later in the paper, we carry out these computations using group cohomology and use the results to compute spectral flow for various arcs of connections of torus bundles over the circle $S^{1}$.

The spectral flow of $D_{A}$ as $A$ varies along a path of connections arises in several ways in topology: If $M$ is a homology 3-sphere, it gives the grading of a flat connection on $M$, viewed as a generator for the chain complex defining Floer homology. It gives the dimension $(\bmod 8)$ of the moduli space of those self-dual connections on $M \times \mathbb{R}$ with certain prescribed limiting values. It also enters in the stationary phase "approximation" of Witten's 3-manifold invariants; the stationary phase formula states that for large $k$ Witten's invariant $Z_{k}=\int_{\mathscr{B}} e^{k i c s}$ is dominated by the sum over the flat connections of terms involving various invariants of the flat connection, in particular the spectral flow to the trivial connection.

Our aim is to give a description of the spectral flow between two flat connections on $M$ when $M$ is cut along a surface $\Sigma \subset M$ in terms

Received September 29, 1992 and, in revised form, November 29, 1993. Both authors supported by NSF Mathematical Sciences Postdoctoral Fellowships. 
of the representation variety $R(M)=\operatorname{Hom}\left(\pi_{1} M, S U(2)\right) /$ conj and the representation varieties of the pieces $X$ and $Y$ where $M X \cup_{\Sigma} Y$. Our main tool is the splitting theorem of [5] and [19], which shows how to decompose the spectral flow into a sum of three terms, one involving only the restriction of $D_{A}$ to $X$, one involving only the restriction of $D_{A}$ to $Y$, and a third term involving the interaction at the boundary.

We now outline the contents of this paper. Section 2 is a review of the results of [4] and [5]. We show how to construct selfadjoint operators on a manifold with boundary in terms of a Lagrangian subspace of the harmonic forms for the "tangential operator". We state the main theorem of [5] which expresses the spectral flow of a path of operators on a closed manifold $M=M_{1} \cup_{\Sigma} M_{2}$ decomposed along a surface $\Sigma$ as a sum of three terms: the spectral flow with respect to a path of Lagrangians $\mathscr{L}_{1}$ on $M_{1}$, the spectral flow with respect to a path of Lagrangians $\mathscr{L}_{2}$ on $M_{2}$, and the Maslov index $\gamma\left(\mathscr{L}_{1}, \mathscr{L}_{2}\right)$.

In $\S 3$ we discuss the properties of the specific family of operators which we are concerned with, namely the operators

$$
D_{A}: \Omega^{0}(M ; s u(2)) \oplus \Omega^{1}(M ; s u(2)) \rightarrow \Omega^{0}\left(M ;{ }^{\prime} s u(2)\right) \oplus \Omega^{1}(M ; s u(2))
$$

on a 3-manifold $M$ and the corresponding tangential operator

$$
\begin{aligned}
\widehat{D}_{\widehat{A}}: & \Omega^{0}(\Sigma ; s u(2)) \oplus \Omega^{1}(\Sigma ; s u(2)) \oplus \Omega^{2}(\Sigma ; s u(2)) \\
& \rightarrow \Omega^{0}(\Sigma ; s u(2)) \oplus \Omega^{1}(\Sigma ; s u(2)) \oplus \Omega^{2}(\Sigma ; s u(2))
\end{aligned}
$$

on $\Sigma=\partial M$. We compute the kernels of these operators and compute the eigenvectors of $\widehat{D}_{\widehat{A}}$ in terms of the eigenvectors of the Laplacian $\Delta_{A}$ on $\Sigma$.

In $\S 4$ we state and prove our main theorem, Theorem 4.8. This theorem expresses the spectral flow of a path $D_{A_{t}}$, when $A_{t}$ is a path of flat connections on a manifold $X$ with boundary such that $\operatorname{ker} D_{A_{t}}=0$ for $t \neq 0$, in terms of the eigenvalues of a bilinear form $B$, namely the composition of the cup product

$$
\text { [ , ]: } H^{1}\left(X, \partial X ; \operatorname{ad} \rho_{t}\right) \times H^{1}\left(X, \partial X ; \operatorname{ad} \rho_{t}\right) \rightarrow H^{2}\left(X, \partial X ; \operatorname{ad} \rho_{t}\right)
$$

and the cup product

$$
\cdot a: H^{2}\left(X, \partial X ; \operatorname{ad} \rho_{t}\right) \rightarrow H^{3}(X, \partial X, \mathbb{R})=\mathbb{R},
$$

where $a$ denotes the 1-dimensional cohomology class defined by the derivative of $\rho_{t}$ at $t=0$, and $\rho_{t}$ denotes the holonomy representation of the flat connection $A_{t}$. Our approach goes as follows: if $A$ is a flat connection on a manifold $X$ with boundary, then the kernel of $D_{A}$ (with 
suitable boundary conditions) can be identified with the cohomology of $X$ with coefficients in the flat bundle determined by the holonomy of $A$. In particular, if $A_{t}$ is a path of flat connections, one can tell by computing cohomology when $D_{A_{t}}$ has kernel. Theorem 4.8 states that the derivatives of the eigenvalues moving through zero are the eigenvalues of $B$ on $H^{1}\left(X, \partial X ; \operatorname{ad} \rho_{t}\right)$. In particular, if these eigenvalues are all nonzero, then the spectral flow equals the signature of this form. Eigenvectors and eigenvalues are studied using the results of analytic perturbation theory [9].

In $\S 5$ we prove the analogous theorem for a closed manifold, Theorem 5.1. We show that if $A_{t}$ is a path of flat connections on a closed manifold, then there is a bilinear form $G$ defined similarly whose eigenvalues are the derivatives of the eigenvalues of $D_{A_{t}}$ which pass through zero at $t=0$.

Theorem 4.8 concerns the computation of spectral flow along an arc of flat connections on a manifold with boundary $X$. In order to understand the spectral flow between two flat connections on a closed manifold decomposed as $M=X \cup Y$, we need to understand the spectral flow along a path of non-flat connections on $Y$, and the interaction term (Maslov index) in the formula of [5]. The approach we take is that the sum of these two terms should define an integer-valued function on the space of paths of representations of $\Sigma$ whose endpoints are representations which extend to $Y$, and in particular the spectral flow should define a homotopy invariant of such paths. Our main interest is in Dehn fillings and surgery on knots, and in $\S 6$ we carry this out for $Y=S^{1} \times D^{2}$. Theorem 6.4 gives an explicit formula for the sum of the spectral flow on $Y$ and the Maslov index in terms of a 1-dimensional cohomology class on (a cover of) the space of representations of $\pi_{1} T$; this formula was implicit in Yoshida's work [20], and we give a more direct proof of a more general version of this formula.

A technical point which arises both in Theorem 4.8 and Theorem 6.3 has to do with reparameterizing the domains of the operators $D_{A}$ on a manifold with boundary. In contrast to the closed case, the domains vary as the connection varies. In Theorem 4.8 a smoothly varying basis of eigenvectors is needed as the path passes through the singularity of $R(X)$. In Theorem 6.4 we need to know that a family of operators parameterized by the representation space of $\Sigma$ forms a continuous family of closed, selfadjoint operators. In Definition 4.5 we say that an analytic path of representations is fine essentially if the domains of the operators can be parameterized nicely. In Theorem 4.8 we assume that the path of flat connections on $X$ is fine. 
We deal with these issues for the torus in Appendix B. Theorem B.1 shows that analytic paths on the torus are fine. Lemma 6.1 stating that the families of operators $D_{A}$ vary continuously with the connection on $D^{2} \times S^{1}$ is also proven in Appendix B; essentially the same ideas are involved in the proof of Lemma 6.1 as in the proof that analytic paths on the torus are fine. Our applications are to Dehn fillings for which the results for the torus suffice. In a later paper we will address these problems for a general surface.

We finish $\S 6$ with an example which shows that the limiting values of extended $L^{2}$-harmonic forms (in the sense of [1]) need not vary continuously along a continuous path of operators, even when the harmonic forms on the boundary vary continuously.

In $\S 7$ we present applications of these results to torus bundles over $S^{1}$. The question which motivated this paper is a conjecture of L. Jeffrey [8] about the spectral flow between two flat connections on a torus bundle. This conjecture arises in trying to reconcile the two main methods of interpreting Witten's 3-manifold invariants, namely the axiomatic TQFT approach and the stationary phase approximation, as we now explain.

In [8], Jeffrey computes the Witten invariants $Z_{k}$ for torus bundles via the TQFT axioms, and then uses the stationary phase formula to compute the "leading term" in the asymptotic expansion as $k \rightarrow \infty$. This leading term is a sum, over the gauge-equivalence classes of flat connections on $M$, of an expression in the Chern-Simons invariants, Reidemeister torsion, and spectral flow. By computing the Chern-Simons invariants and the Reidemeister torsion, Jeffrey gives an formula involving the spectral flow which must hold if the two interpretations of Witten's invariant are to be consistent. This formula implies that the spectral flow between any two nonabelian flat $S U(2)$-connections on a torus bundle is congruent to 0 $\bmod 4$.

In Theorem 7.4 we show that under mild restrictions this is indeed the case, and we use this theorem to compute several examples. The beginning observation is Corollary 7.2, which states that given any two irreducible $S U(2)$ representations $\rho_{0}, \rho_{1}$ of a torus bundle $M$ over $S^{1}$, there exist a knot $K$ in $M$ and a path of representations $\rho_{t}$ of $\pi_{1}(M-K)$ joining $\rho_{0}$ to $\rho_{1}$. By computing group cohomology one sees when the kernel jumps up, i.e., when an eigenvalue crosses zero. Applying Theorem 4.8 and Lemma 6.4 reduces the computation of $S F\left(\rho_{0}, \rho_{1} ; M\right)$ to computing the signature of a bilinear form on cohomology induced by the cup products. We then use Theorem 5.1 to compute the spectral flow between certain abelian representations of torus bundles. 
We list in $\S 8$ some questions which we were unable to resolve. These are mostly of a technical nature, and their solutions would allow us to remove some of the hypotheses for Theorem 4.8, Lemma 6.4, and Definition 7.4.

We would like to thank D. Hoff for helpful conversations.

\section{Spectral flow on a 3-manifold split along a surface}

Let $M$ be a smooth closed oriented 3-manifold, with $\Sigma \subset M$ a smooth embedded separating surface. Let $M_{1}$ and $M_{2}$ be the closures of the complementary components of $M-\Sigma$, so $M=M_{1} \cup_{\Sigma} M_{2}$. Fix an embedding $i: \Sigma \times[-1,1] \rightarrow M$ such that $i(\sigma, 0)=\sigma$ for $\sigma \in \Sigma$. Put a Riemannian metric $g$ on $M$ such that $i$ is an isometry for some metric on $\Sigma$. Using $i$, identify $\Sigma \times[-1,1]$ with its image in $M$. Let $\pi: \Sigma \times[-1,1] \rightarrow \Sigma$ be projection.

Let $E \rightarrow M$ be a real vector bundle with a fiberwise inner product $x \cdot y$. Assume we are given an identification of $E \mid(\Sigma \times[-1,1])$ with $\pi^{*}(\widehat{E})$, where $\widehat{E} \rightarrow \Sigma$ is a real vector bundle. Assume this identification is compatible with a fiberwise inner product on $\widehat{E}$, which we also denote by $x \cdot y$. These inner products give rise to inner products on $\Gamma(E)$ and $\Gamma(\widehat{E})$ defined by

$$
\langle f, g\rangle_{M}=\int_{M} f \cdot g \text { dvol, } \quad\langle f, g\rangle_{\Sigma}=\int_{\Sigma} f \cdot g \text { dvol }
$$

The words "selfadjoint" will always refer to these inner products.

Let $\sigma: \widehat{E} \rightarrow \widehat{E}$ be a bundle automorphism, and let $\pi^{*} \sigma$ be its pullback to $E \mid(\Sigma \times[-1,1])$. Let $D: \Gamma(E) \rightarrow \Gamma(E)$ be a first order selfadjoint elliptic operator. Assume that

$$
D=\left(\pi^{*} \sigma\right) \circ\left(\frac{\partial}{\partial s}+\pi^{*} \widehat{D}\right)
$$

over $\Sigma \times[-1,1]$, where $\widehat{D}$ is a first-order selfadjoint elliptic operator on $\Gamma(\widehat{E}$ ) (called the tangential operator), and $s$ is the coordinate on $[-1,1]$. From the above (Proposition 2.A of [4]) we have the following:

(i) $\sigma^{*}=-\sigma$ and $\sigma \widehat{D}=-\widehat{D} \sigma$ on $\Sigma$.

(ii) There is a nondegenerate symplectic pairing on $L^{2}(\widehat{E})$ given by $\{f, g\}=\langle f, \sigma g\rangle_{\Sigma}$.

(iii) Let $\mathscr{H}=\operatorname{ker} \widehat{D}$. The restriction to $\mathscr{H}$ of the symplectic pairing in (ii) is a nondegenerate symplectic pairing. 
Let $\left\{\alpha_{k}\right\} \subset \Gamma(\widehat{E})$ be a complete orthonormal basis of $L^{2}(\widehat{E})$ with

$$
\widehat{D} \alpha_{k}=\nu_{k} \alpha_{k} \text { for each } k
$$

with all $\nu_{k}$ 's real. Define

$$
\begin{aligned}
& P_{+}=L^{2}-\text { closure of }\left\{\alpha_{k} \mid \nu_{k}>0\right\}, \\
& P_{-}=L^{2}-\text { closure of }\left\{\alpha_{k} \mid \nu_{k}<0\right\} .
\end{aligned}
$$

By (i) above, $\sigma\left(P_{+}\right)=P_{-}$and $\sigma\left(P_{-}\right)=P_{+}$. Also, $L^{2}(\widehat{E})=P_{+} \oplus P_{-} \oplus \mathscr{H}$. Define $E_{i}=E \mid M_{i}$. Let $D^{i}=D \mid \Gamma\left(E_{i}\right)$, for $i=1,2$. If $V$ is a subspace of $L^{2}(\widehat{E})$, define $L_{1}^{2}\left(E_{j} ; V\right)$ to be the Sobolev $L_{1}^{2}$-completion of those smooth sections $\varphi$ of $E_{j}$ satisfying $\varphi \mid \Sigma \in V$.

Let $\mathscr{L} \subset \mathscr{H}$ be a Lagrangian. Define $\mathscr{D}^{j}$ to be the restriction of $D$ to the image of $L_{1}^{2}\left(E_{j} ; P_{+}+\mathscr{L}\right)$ in $L^{2}\left(E_{j}\right)$. Then by Proposition 2.D, [4], $\mathscr{D}^{j}$ is a selfadjoint elliptic operator.

We note here that the operators $\widehat{D}$ and $\mathscr{D}^{j}$ both are Fredholm and have compact resolvents. In the case of $\widehat{D}$, these are well-known properties of an elliptic operator on a closed manifold. The fact that $\mathscr{D}^{j}$ is Fredholm is proven in [1, p. 55]. The fact that $\mathscr{D}^{j}$ has compact resolvent follows because its Green's operator from $L^{2}\left(E_{j}\right)$ to $L_{1}^{2}\left(E_{j} ; P_{+}+\mathscr{L}\right)$ is bounded and the inclusion $L_{1}^{2}\left(E_{j}\right) \rightarrow L^{2}\left(E_{j}\right)$ is compact (see [15, p. 168]).

We will also need the following stretched versions of $M$. Let

$$
\begin{aligned}
M(r) & =M_{1} \cup(\Sigma \times[-r, r]) \cup M_{2}, \\
M_{1}(r) & =M_{1} \cup(\Sigma \times[-r, 0]), \\
M_{2}(r) & =(\Sigma \times[0, r]) \cup M_{2} .
\end{aligned}
$$

We extend our bundle $E$ to $E(r)$ over $M(r), E_{1}(r)$ over $M_{1}(r), E_{2}(r)$ over $M_{2}(r)$, and define inner products on these extended bundles using the pullback of $\widehat{E}$ by $\pi: \Sigma \times[-r, r] \rightarrow \Sigma$.

We extend our operator $D$ to sections of these bundles by keeping it the same over $M_{1}$ and $M_{2}$ and defining it over $\Sigma \times[-r, r]$ by the formula

$$
D=\pi^{*} \sigma \circ\left(\pi^{*} \widehat{D}+\frac{\partial}{\partial s}\right) \text {. }
$$

Our main tool will be the splitting theorem for spectral flow. There are various versions of this theorem; see [5], [19], [14], and [13].

Suppose we are given one-parameter families $D_{t}$ and $\widehat{D}_{t}, t \in[0,1]$, of first-order selfadjoint elliptic operators related to each other for each $t$ by the formula above. In addition, suppose $\mathscr{H}(t)=\operatorname{ker} \widehat{D}_{t}$ has dimension 
independent of $t$, and hence forms a symplectic vector bundle over the interval $t \in[0,1]$.

Let $\mathscr{L}_{1}(t)$ and $\mathscr{L}_{2}(t)$ be continuous families of Lagrangians in the symplectic spaces $\mathscr{H}(t)$.

We need to assume some nondegeneracy conditions at the endpoints of the interval. We first assume that there are no nonzero $L^{2}$ solutions to the equations $D_{0}^{i} \phi=0$ and $D_{1}^{i} \phi=0, i=1,2$, on the manifolds obtained by adding an infinite cylinder $\Sigma \times[0, \infty)$ to $M_{i}$, for $i=1,2$. Equivalently, the operators $D_{0}^{i}$ and $D_{1}^{i}, i=1,2$, have no solutions with $P_{+}$boundary conditions in the sense of [1]. This nondegeneracy condition is called nonresonance in [14].

Recall also from [1] that there are natural Lagrangian subspaces $L_{0}^{i} \subset$ $\mathscr{H}(0)$ and $L_{1}^{i} \subset \mathscr{H}(1)$, namely the limiting values of extended $L^{2}$ solutions. We will require that $\mathscr{L}_{i}(0)=\sigma L_{0}^{i}$ and $\mathscr{L}_{i}(1)=\sigma L_{1}^{i}$. In other words, we assume that the families of Lagrangians $\mathscr{L}_{i}(t)$ start and end at the complementary Lagrangians to the natural Lagrangians. We will say that the paths $\mathscr{L}_{i}(t)$ of Lagrangians are admissible if they satisfy these properties.

Let

$$
\mathscr{D}_{t}^{j}(r)=D_{t} \mid L_{1}^{2}\left(E_{j}(r) ; P_{+}(t)+\mathscr{L}_{j}(t)\right) .
$$

Notice that the conditions of the preceding paragraphs imply that the kernels of $\mathscr{D}_{t}^{j}(r)$ are zero at the endpoints. Then the following is a version of the main theorem of [5] (see also [19], [14], and [13]).

Theorem 2.1. Assume $D_{0}^{i}$ and $D_{1}^{i}$ for $i=1,2$ are nonresonant, the paths $\mathscr{L}_{i}(t)$ are admissible, and $L_{0}^{1} \cap L_{0}^{2}=0=L_{1}^{1} \cap L_{1}^{2}$. Then, for large enough $r$,

$$
S F\left(D_{t}\right)=S F\left(\mathscr{D}_{t}^{1}(r)\right)+S F\left(\mathscr{D}_{t}^{2}(r)\right)+\gamma\left(\mathscr{L}_{1}(t), \mathscr{L}_{2}(t)\right),
$$

where $S F$ is spectral flow of a family of selfadjoint operators, and $\gamma$ is the Maslov index of two paths of Lagrangians in the family $\mathscr{H}(t)$ of symplectic spaces.

(We remind the reader that the spectral flow $S F\left(D_{t}\right)$ of a path of selfadjoint operators is the integer which counts the number of eigenvalues crossing from negative to positive minus the number crossing from positive to negative. See [1].)

This theorem has been used to compute the spectral flow in certain cases where $S F\left(\mathscr{D}_{t}^{1}\right)=0$ (see [19], [20]). Theorem 4.8 gives a method for computing $S F\left(\mathscr{D}_{t}^{1}\right)$ in certain cases when it is not 0 , thereby considerably increasing the usefulness of this theorem. 


\section{The Hessian of the Chern-Simons function}

We now focus our attention on a particular class of bundles and operators which arise in the computation of Floer homology [7], and in the stationary phase approximation of Witten's invariants [18], namely the Hessian of the Chern-Simons functions on the space of gauge equivalence classes of connections (actually the operators we consider are stabilizations of the Hessian; i.e., the direct sum of the Hessian with an operator with symmetric spectrum.) These operators were treated extensively by Yoshida in [19].

In this section, $\Sigma$ and $M$ are as in $\S 2$. Define the bundles $E \rightarrow M$ and $\widehat{E} \rightarrow \Sigma$ by

$$
E=\left(\Lambda^{0} T^{*} M \oplus \Lambda^{1} T^{*} M\right) \otimes s u(2)
$$

and

$$
\widehat{E}=\left(\Lambda^{0} \oplus \Lambda^{1} \oplus \Lambda^{2}\right) T^{*} \Sigma \otimes s u(2)
$$

Thus

$$
\Gamma(E)=\Omega^{0}(M ; s u(2)) \oplus \Omega^{1}(M ; s u(2))=\left(\Omega^{0} \oplus \Omega^{1}\right)(M ; s u(2))
$$

and

$$
\Gamma(\widehat{E})=\left(\Omega^{0} \oplus \Omega^{1} \oplus \Omega^{2}\right)(E ; s u(2)) .
$$

We identify $\widehat{E}_{\sigma}$ with $E_{\left(\sigma, s_{0}\right)}$ by $(\alpha, \beta, \gamma) \mapsto(\alpha, \beta+(* \gamma) \wedge d s)$ where * acts as the usual Hodge star operator $\Lambda^{i} T^{*} \Sigma \rightarrow \Lambda^{2-i} T^{*} \Sigma$ and by the identity on $s u(2)$. It is clear that this map is an isomorphism on each fiber; we take it to give an identification of $E \mid\left(\Sigma \times[-1,1] \cong \pi^{*} \widehat{E}\right.$.

Let $A$ be a connection on $M \times S U(2)$, which we think of as an element of $\Omega^{1}(M ; s u(2))$. We will always assume that on $\Sigma \times[-1,1], A=\pi^{*} \hat{A}$, where $\widehat{A}$ is a connection (i.e., 1 -form) on $\Sigma \times S U(2)$. These connections give rise to exterior derivatives

$$
d_{A}: \Omega^{*}(M ; s u(2)) \rightarrow \Omega^{*+1}(M ; s u(2))
$$

and

$$
d_{\widehat{A}}: \Omega^{*}\left(\Sigma ; s u(2) \rightarrow \Omega^{*+1}(\Sigma ; s u(2))\right.
$$

Also, $d_{A} \circ d_{A}=0$ (respectively, $d_{\widehat{A}} \circ d_{\widehat{A}}=0$ ) if and only if $A$ (respectively $\widehat{A})$ is flat.

Define $\widehat{D}_{\widehat{A}}: \Gamma(\widehat{E}) \rightarrow \Gamma(\widehat{E})$ as follows:

$$
\widehat{D}_{\widehat{A}}(\alpha, \beta, \gamma)=\left(* d_{\widehat{A}} \beta,-* d_{\widehat{A}} \alpha-d_{\widehat{A}} * \gamma, d_{\widehat{A}} * \beta\right) .
$$

Define $D_{A}: \Gamma(E)^{-} \rightarrow \Gamma(E)$ by

$$
D_{A}(\sigma, \tau)=\left(d_{A}^{*} \tau, d_{A} \sigma+* d_{A} \tau\right)
$$


We adopt the sign conventions of Warner [17]: $d^{*}: \Omega^{p}\left(M^{n}\right) \rightarrow \Omega^{p-1}\left(M^{n}\right)$ is defined by

$$
d^{*}=(-1)^{n(p+1)+1} * d * \text { and } * *=(-1)^{p(n-p)}
$$

on $\Omega^{p}\left(M^{n}\right)$. Also $\Delta^{p}=d^{*} d+d d^{*}$ on $\Omega^{p}\left(M^{n}\right)$.

Our bundle automorphism $\sigma: \widehat{E} \rightarrow \widehat{E}$ is defined by $\sigma(\alpha, \beta, \gamma)=(-*$ $\gamma, * \beta, * \alpha)$. Using these operators, we have:

(1) $D_{A}=\pi^{*} \sigma \circ\left(\partial / \partial s+\pi^{*} \widehat{D}_{\widehat{A}}\right)$ on $\Sigma \times[-1,1]$.

(2) $\left(\widehat{D}_{\widehat{A}}\right)^{2}=\Delta_{\widehat{A}}^{0} \oplus \Delta_{\widehat{A}}^{1} \oplus \Delta_{\widehat{A}}^{2}$ if $\hat{A}$ is flat, and $\left(D_{A}\right)^{2}=\Delta_{A}^{0} \oplus \Delta_{A}^{1}$ if $A$ is flat.

(3) $\widehat{D}_{\widehat{A}}$ and $D_{A}$ are first-order elliptic selfadjoint operators; in fact they are operators of Dirac type.

We explain the context in which this operator arises, following Taubes [16]. If $A$ is a connection on a closed 3-manifold $M$, and $K_{A}=$ $\operatorname{ker} d_{A}^{*}: \Omega^{1}(M) \otimes s u(2) \rightarrow \Omega^{0}(M) \otimes s u(2)$, then the Hessian of the ChernSimons function at $A$ is the operator $H_{A}=\operatorname{proj}_{K_{A}} * d_{A}$ (note that all projections are with respect to the $L^{2}$ norm). Decompose $\Omega^{0}(M) \otimes s u(2) \oplus$ $\Omega^{1}(M) \otimes s u(2)$ as $\Omega^{0}(M) \otimes s u(2) \oplus K_{A}^{\perp} \oplus K_{A}$. Let $B_{A}$ be the operator

$$
B_{A}=\left(\begin{array}{cc} 
& d_{A}^{*} \\
d_{A} &
\end{array}\right)
$$

on $\Omega^{0}(M) \otimes s u(2) \oplus K_{A}^{\perp}$. Then $B_{A}$ is selfadjoint and has a symmetric spectrum. Moreover, $B_{A} \oplus H_{A}$ equals $D_{A}$ when $A$ is flat, and is a compact perturbation of $D_{A}$ in general. Thus if $A_{t}$ is a path of connections with $A_{0}$ and $A_{1}$ flat, the spectral flow of $H_{A_{t}}$ equals the spectral flow of $H_{A_{t}} \oplus B_{A_{t}}$, which in turn equals the spectral flow of $D_{A_{t}}$.

Assume from now on that the connection $\widehat{A}$ on $\Sigma \times S U(2)$ is flat. Then

$$
\mathscr{H}=\operatorname{ker} \widehat{D}_{\widehat{A}} \cong H_{\widehat{A}}^{0}(\Sigma) \oplus H_{\widehat{A}}^{1}(\Sigma) \oplus H_{\widehat{A}}^{2}(\Sigma)
$$

by Hodge theory. (The cohomology groups come from the complex $\Omega^{*}(\Sigma ; s u(2))$ using the differential $d_{\widehat{A}}$.) By abuse of notation, we will often use $H_{\widehat{A}}^{i}(\Sigma)$ to denote these harmonic $i$-forms. Using the DeRham theorem these groups are also equal to $H^{i}\left(\Sigma ;\right.$ ad $\left.\rho_{\widehat{A}}\right)$, where $\rho_{\widehat{A}}: \pi_{1} \Sigma \rightarrow$ $S U$ (2) denotes the holonomy representation of the flat connection $\widehat{A}$, and ad $\rho_{\widehat{A}}$ is shorthand for the local coefficients given by the action of $\pi_{1} \Sigma$ on the Lie algebra $s u(2)$ via the adjoint representation ad: $S U(2) \rightarrow$ $\operatorname{Aut}(s u(2))$. 
The inner product on $E$ (and $\widehat{E})$ is given by $x \cdot y=-\operatorname{tr}(x \wedge * y) /(\mathrm{dvol})$ for $x, y \in\left(\Lambda^{i} T^{*} M\right)_{p}$. Hence the inner product on $\Gamma(E)$ is given by

$$
\langle x, y\rangle_{M}=-\int_{M} \operatorname{tr}(x \wedge * y),
$$

and similarly for $\Sigma$.

It follows that the symplectic inner product on $\Gamma(\widehat{E})$ is given by

$$
\begin{aligned}
& \{x, y\}=\int_{\Sigma} \operatorname{tr}(x \wedge y) \quad \text { if }(\operatorname{dim} x, \operatorname{dim} y)=(0,2) \text { or }(1,1), \\
& \{x, y\}=-\int_{\Sigma} \operatorname{tr}(x \wedge y) \quad \text { if }(\operatorname{dim} x, \operatorname{dim} y)=(2,0) \\
& \{x, y\}=0 \text { if } \operatorname{dim} x+\operatorname{dim} y \neq 2 .
\end{aligned}
$$

We will need some facts about the eigenfunctions of $\widehat{D}_{\widehat{A}}$ on $\Gamma(\widehat{E})$. Let $H^{0}(\Sigma)$ denote the harmonic 0 -forms. Because $\Delta_{\widehat{A}}$ is a nonnegative selfadjoint operator, we may write $L^{2}\left(\Omega^{0}(\Sigma ; s u(2))\right)=H_{\widehat{A}}^{0}(\Sigma) \oplus \operatorname{span}\left\{\varphi_{k}\right\}_{k=1}^{\infty}$, where $\Delta_{\widehat{A}} \varphi_{k}=\mu_{k} \varphi_{k}, \mu_{k}>0$ for each $k$. We also assume that $\left|\varphi_{k}\right|=1$ for all $k$. Using the $\varphi_{k}$, we will explicitly construct the eigenfunctions of $\widehat{D}_{\widehat{A}}$ on $\Gamma(\widehat{E})$.

For each $k$, define the following four elements of $\Gamma(\widehat{E})$ :

$$
\begin{array}{lll}
\xi_{k}^{+}=\left(\varphi_{k},-\frac{1}{\sqrt{\mu_{k}}} * d_{\widehat{A}} \varphi_{k}, 0\right), & \psi_{k}^{+}=\left(0,-\frac{1}{\sqrt{\mu_{k}}} d_{\widehat{A}} \varphi_{k}, * \varphi_{k}\right), \\
\xi_{k}^{-}=\left(\varphi_{k}, \frac{1}{\sqrt{\mu_{k}}} * d_{\widehat{A}} \varphi_{k}, 0\right), & \psi_{k}^{-}=\left(0, \frac{1}{\sqrt{\mu_{k}}} d_{\widehat{A}} \varphi_{k}, * \varphi_{k}\right) .
\end{array}
$$

\section{Proposition 3.1.}

$$
L^{2}(\widehat{E})=\left(H_{\widehat{A}}^{0} \oplus H_{\widehat{A}}^{1} \oplus H_{\widehat{A}}^{2}\right)(\Sigma) \oplus \operatorname{span}\left\{\xi_{k}^{+}, \xi_{k}^{-}, \psi_{k}^{+}, \psi_{k}^{-}\right\}_{k=1}^{\infty},
$$

where $\xi_{k}^{+}$and $\psi_{k}^{+}$are $\sqrt{\mu_{k}}$-eigenvectors of $\widehat{D}_{\widehat{A}}, \xi_{k}^{-}$and $\psi_{k}^{-}$are $-\sqrt{\mu_{k}}$ eigenvectors of $\hat{D}_{\widehat{A}},\left|\xi_{k}^{+}\right|=\left|\xi_{k}^{-}\right|=\left|\psi_{k}^{+}\right|=\left|\psi_{k}^{-}\right|=\sqrt{2}$; and all of these eigenvectors are mutually orthogonal.

Proof. Everything is a straightforward computation except (perhaps!) completeness. To see this, note

$$
\begin{aligned}
& \operatorname{span}\left\{\xi_{k}^{+}+\xi_{k}^{-}\right\}_{k} \oplus H_{\vec{A}}^{0}(\Sigma)=L^{2}\left(\Omega^{0}(\Sigma)\right), \\
& \operatorname{span}\left\{\psi_{k}^{+}+\psi_{k}^{-}\right\}_{k} \oplus H_{\vec{A}}^{2}(\Sigma)=L^{2}\left(\Omega^{2}(\Sigma)\right), \\
& \operatorname{span}\left\{\xi_{k}^{+}-\xi_{k}^{-}\right\}_{k}=\operatorname{im}\left(d^{*}\right) \subset L^{2}\left(\Omega^{1}(\Sigma)\right), \\
& \operatorname{span}\left\{\psi_{k}^{+}-\psi_{k}^{-}\right\}_{k}=\operatorname{im}(d) \subset L^{2}\left(\Omega^{1}(\Sigma)\right) .
\end{aligned}
$$

By Hodge theory, $L^{2}\left(\Omega^{1}(\Sigma)\right)=\operatorname{im}\left(d^{*}\right) \oplus \operatorname{im}(d) \oplus H_{\widehat{A}}^{1}(\Sigma)$. 


\section{Spectral flow along arcs of flat connections on a 3-manifold with boundary}

In this section we state and prove our main theorem. It really only involves $M_{1}$; to avoid subscripts we change notation as follows:

Let $X$ be a compact oriented 3-manifold with $\partial X=\Sigma$. Assume $X$ has a Riemannian metric such that a collar of $\Sigma$ is isometric to (and identified with) $\Sigma \times[-1,0]$, with $\Sigma \times\{0\}=\partial X$. Let

$$
\begin{aligned}
X(\infty) & =X \cup(\Sigma \times[0, \infty)), \\
X(r) & =X \cup(\Sigma \times[0, r]), \quad r \in \mathbb{R}_{\geq 0} .
\end{aligned}
$$

As before let $E(r) \rightarrow X(r)$ be the bundle $\left(\Lambda^{0} T^{*} \oplus \Lambda^{1} T^{*}\right)(X(r)) \otimes s u(2)$ and let $\widehat{E} \rightarrow \Sigma$ be as defined in the previous section. In this section we will always assume that $A$ is a flat connection on $X(\infty) \times S U(2)$, and that on $\Sigma \times[-1, \infty), A=\pi^{*} \widehat{A}$ where $\hat{A}$ is a flat connection on $\Sigma \times S U(2)$. We then define the operators $D_{A}$ and $\widehat{D}_{\widehat{A}}$ on $\Gamma(E(\infty))$ and $\Gamma(\widehat{E})$ as in $\S 3$.

Let $L_{1, \text { loc }}^{2}(E(\infty))$ denote those sections of $E(\infty)$ which are locally $L_{1}^{2}$.

Definition 4.1 [1]. Let $\varphi \in L_{1, \text { loc }}^{2}(E(\infty))$. We say $\varphi$ is an extended $L^{2}$-section if there is a section $\eta \in L^{2}(\widehat{E})$ such that $(\varphi \mid \Sigma \times[0, \infty))-\pi^{*}(\eta) \epsilon$ $L^{2}(\Sigma \times[0, \infty))$. In this case, we say $\eta$ is the limiting value of $\varphi$.

We denote the set of extended $L^{2}$-sections of $E(\infty)$ which are also in $L_{1, \text { loc }}^{2}$ by $\left.\widetilde{L}_{1}^{2}(\infty)\right)$.

Denote by $\left\{\alpha_{k}\right\}$ an orthonormal basis of $L^{2}(\widehat{E})$ with $\widehat{D}_{\widehat{A}} \alpha_{k}=\nu_{k} \alpha_{k}$ for all $k$. It is clear that if $\varphi \in L_{1, \text { loc }}^{2}(X(\infty))$ and $D_{A} \varphi=0$, then $\varphi \mid(\Sigma \times[0, \infty))$ is of the form

$$
\varphi=\sum_{k=1}^{\infty} c_{k} e^{-\nu_{k} s} \alpha_{k} .
$$

It follows that if $D_{A} \varphi=0$, then

(i) $\varphi \in L_{1}^{2}(E(\infty)) \Leftrightarrow c_{k}=0$ whenever $\nu_{k} \leq 0$,

(ii) $\varphi \in \widetilde{L}_{1}^{2}(E(\infty)) \Leftrightarrow c_{k}=0$ whenever $\nu_{k}<0$.

Define

$$
V=\operatorname{ker}\left(D_{A} \mid L_{1}^{2}(E(\infty)) \quad \text { and } \quad \widetilde{V}=\operatorname{ker}\left(D_{A} \mid \widetilde{L}_{1}^{2}(E(\infty))\right)\right.
$$

We now have the following important facts: for all $r \geq 0$,

$$
V \cong \operatorname{ker}\left(D_{A} \mid L_{1}^{2}\left(E(r) ; P_{+}\right)\right) \quad \text { and } \tilde{V} \cong \operatorname{ker}\left(D_{A} \mid L_{1}^{2}\left(E(r) ; P_{+}+\mathscr{H}\right)\right) \text {, }
$$

where these isomorphisms are obtained by restricting sections. 
Suppose $\varphi \in \widetilde{V}$; then on $\Sigma \times[0, \infty)$ we write

$$
\varphi=\alpha+\sum_{\nu_{k}>0} c_{k} e^{-\nu_{k} s} \alpha_{k},
$$

where $\alpha \in \operatorname{ker} \widehat{D}_{\widehat{A}}=\mathscr{H}$. In this expression $\alpha$ is the limiting value of $\varphi$.

Proposition 4.2. Let

$$
(x, y) \in \widetilde{V} \subset \widetilde{L}_{1}^{2}\left(\Omega^{0} \oplus \Omega^{1}\right)(X(\infty) ; s u(2)) .
$$

Then $d_{A} x=d_{A}^{*} y=d_{A} y=0$ (i.e., $x$ and $y$ are harmonic).

Proof. By definition of $D_{A}$, we know $* d_{A} y=-d_{A} x$ which implies that $d_{A}^{*} d_{A} x=0$. On $\Sigma \times[0, \infty)$, we can write

$$
(x, y)=\left(\alpha^{0}, \alpha^{1}, \alpha^{2}\right)+\sum_{\nu_{k}>0} c_{k} e^{-\nu_{k} s}\left(\alpha_{k}^{0}, \alpha_{k}^{1}, \alpha_{k}^{2}\right),
$$

where $\alpha=\left(\alpha^{0}, \alpha^{1}, \alpha^{2}\right)$ is a harmonic $(0+1+2)$-form, and $\alpha_{k}=$ $\left(\alpha_{k}^{0}, \alpha_{k}^{1}, \alpha_{k}^{2}\right)$ is a $\nu_{k}$-eigenvector of $\widehat{D}_{\widehat{A}}$. In particular,

$$
x=\alpha^{0}+\sum_{\nu_{k}>0} c_{k} e^{-\nu_{k} s} \alpha_{k}^{0} .
$$

We claim $d_{A} x$ decays exponentially as $s \rightarrow \infty$. To see this, use the fact that $d_{A}=d_{\widehat{A}}+\partial() / \partial s \wedge d s$ on $\Omega^{0}(X(\infty), s u(2))$ to write

$$
d_{A} x=\sum_{\nu_{k}>0} c_{k} e^{-\nu_{k} s}\left(-\nu_{k} \alpha_{k}^{0} \wedge d s+d_{\widehat{A}} \alpha_{k}^{0}\right)
$$

which clearly decays exponentially in $s$.

Compute:

$$
\begin{aligned}
& \left|d_{A} x\right|_{X(\infty)}^{2} \\
& \quad=\lim _{r \rightarrow \infty}\left|d_{A} x\right|_{X(r)}^{2}=\lim _{r \rightarrow \infty}\left(\left\langle x, d_{A}^{*} d_{A} x\right\rangle_{X(r)}+\left\langle x, d_{\widehat{A}} x\right\rangle_{\Sigma \times\{r\}}\right)=0,
\end{aligned}
$$

where the first equality is by the monotone convergence theorem, the second is by Stokes' theorem, and the third is because $x$ is bounded and $d_{A} x$ decays exponentially as $s \rightarrow \infty$. Therefore, $d_{A} x=0$ and the other assertions follow immediately. q.e.d.

Define $p: \widetilde{V} \rightarrow \mathscr{H}$ by $p(\varphi)=\lim _{s \rightarrow \infty}\left(\left.\varphi\right|_{\Sigma \times\{s\}}\right)$, in other words, $p(\varphi)$ is defined to be the limiting value of $\varphi$. (Equivalently, we could define $p(\varphi)=p_{\mathscr{H}}\left(\left.\varphi\right|_{\Sigma \times\{s\}}\right)$ for any $s \geq 0$, where $p_{\mathscr{H}}: L^{2}(\Sigma) \rightarrow \mathscr{H}$ is orthogonal projection.) Notice that $H_{\widehat{A}}^{0}(\Sigma)=0=H_{\widehat{A}}^{2}(\Sigma)$ if $\widehat{A}$ is irreducible. (The second equality follows from the Poincaré duality.) 


\section{Proposition 4.3.}

(I) If $A$ and $\widehat{A}$ are both irreducible, then

$$
p(\widetilde{V})=\operatorname{im}\left(H_{A}^{1}(X) \rightarrow H_{\widehat{A}}^{1}(\Sigma)\right) .
$$

(II) If $A$ is irreducible and $\widehat{A}$ is reducible, then

$$
p(\tilde{V})=\operatorname{im}\left(H_{A}^{1}(X) \rightarrow H_{\widehat{A}}^{1}(\Sigma)\right) \oplus H_{\widehat{A}}^{2}(\Sigma) .
$$

(III) If $A$ and $\hat{A}$ are both reducible, then

$$
p(\tilde{V})=H_{\widehat{A}}^{0}(\Sigma) \oplus \operatorname{im}\left(H_{A}^{1}(X) \rightarrow H_{\widehat{A}}^{1}(\Sigma)\right) .
$$

Proof. Let $(x, y) \in \widetilde{V}$. On $\Sigma \times[0, \infty)$ we write

$$
(x, y)=\left(\alpha^{0}, \alpha^{1}, \alpha^{2}\right)+\sum_{K=1}^{\infty} e^{-\sqrt{\mu_{k}} s}\left(a_{k} \xi_{k}^{+}+b_{k} \psi_{k}^{+}\right) \text {. }
$$

Then

$$
p(x, y)=\left(\alpha^{0}, \alpha^{1}, \alpha^{2}\right) \in\left(H_{\widehat{A}}^{0} \oplus H_{\widehat{A}}^{1} \oplus H_{\widehat{A}}^{2}\right)(\Sigma) .
$$

Let $i: \Sigma \times\{0\} \hookrightarrow X(\infty)$ be the inclusion. Since $d_{A} x=0$, we know $d_{\widehat{A}}\left(i^{*} x\right)=0$ so $i^{*} x$ is harmonic. But $i^{*} x=\alpha^{0}+\Sigma a_{k} \varphi_{k}$. Since the $\varphi_{k}$ are orthogonal to the harmonic 0 -forms, $a_{k}=0$ for all $k$. Thus $(x, y)=\left(\alpha^{0}, \alpha^{1}, \alpha^{2}\right)+\sum_{k=1}^{\infty} b_{k} e^{-\sqrt{\mu_{k}} s} \psi_{k}^{+}$on $\Sigma \times[-1, \infty)$. Since $i^{*} y=$ $\alpha^{1}+\sum_{k=1}^{\infty} b_{k}\left(-1 / \sqrt{\mu_{k}}\right) d_{\widehat{A}} \varphi_{k}, \alpha^{1}=i^{*}(y)$ in $H_{\widehat{A}}^{1}(\Sigma)$.

Suppose $A$ and $\widehat{A}$ are both irreducible. Then $H_{\widehat{A}}^{0}(\Sigma)=H_{\widehat{A}}^{2}(\Sigma)=0$, so $\alpha^{0}=\alpha^{2}=0$. Thus $p(x, y)=\alpha^{1}=i^{*} y \in \operatorname{im}\left(H_{A}^{1}(X) \rightarrow H_{\widehat{A}}^{1}(\Sigma)\right)$. Since $\operatorname{dim} p(\widetilde{V})=\frac{1}{2} \operatorname{dim} H_{\widehat{A}}^{1}(\Sigma)$ (by Proposition 2.C in [4]; see also [19]), $\operatorname{dim} p(\tilde{V})=\operatorname{im}\left(H_{A}^{1} X \rightarrow H_{\widehat{A}}^{1} \Sigma\right)$.

Suppose $A$ and $\hat{A}$ are both reducible but non-central. Then $H_{A}^{0}(X)=$ $H_{\widehat{A}}^{0} \Sigma=H_{\widehat{A}}^{2} \Sigma=\mathbb{R}$. Thus $p(\widetilde{V}) \supset H_{\widehat{A}}^{0} \Sigma$, (since every covariantly constant section of $\Sigma \times s u(2)$ extends to one of $X \times s u(2)$.

It follows that $p(\widetilde{V}) \subset H_{\widehat{A}}^{0} \Sigma \oplus H_{\widehat{A}}^{1} \Sigma$ because $p(\widetilde{V})$ is a Lagrangian. Finally, since $p(x, y)=\left(\alpha^{0}, \alpha^{1}, 0\right)$ and we know $\alpha^{1} \in \operatorname{im}\left(H_{A}^{1} X \rightarrow H_{\widehat{A}}^{1} \Sigma\right)$, $p(\widetilde{V})=Y_{\widehat{A}}^{0} \Sigma \oplus \operatorname{im}\left(H_{\widehat{A}}^{1} X \rightarrow H_{\widehat{A}}^{1} \Sigma\right)$ by a dimension argument.

Finally, suppose $A$ is irreducible but $\widehat{A}$ is reducible. Because $d_{A} x=0$, $x=0$ so $\alpha^{0}=0$. It follows that $p(\tilde{V}) \subset H_{\widehat{A}}^{1} \Sigma \oplus H_{\widehat{A}}^{2} \Sigma$. Since $p(x, y)=$ $\left(0, \alpha^{1}, \alpha^{2}\right)$ and $\alpha^{1} \in \operatorname{im}\left(H_{\widehat{A}}^{1} X \rightarrow H_{\widehat{A}}^{1} \Sigma\right)$, a dimension argument shows

$$
p(\tilde{V})=\operatorname{im}\left(H_{A}^{1} X \rightarrow H_{\widehat{A}}^{1} \Sigma\right) \oplus H_{\widehat{A}}^{2} \Sigma \text {. q.e.d. }
$$


Observe that $\operatorname{ker}(p)=L^{2}$ harmonic $(0+1)$-forms on $X(\infty)$. It follows from Proposition 4.9 of [1] that

$$
\operatorname{ker} p=\operatorname{im}\left(H_{A}^{1}(X, \Sigma) \rightarrow H_{A}^{1} X\right) \oplus \operatorname{im}\left(H_{A}^{0}(X, \Sigma) \rightarrow H_{A}^{0} X\right) ;
$$

since the second summand is always 0 , we have $\operatorname{ker}(p)=\operatorname{im}\left(H_{A}^{1}(X, \Sigma) \rightarrow\right.$ $\left.H_{A}^{1} X\right)$. Let $\mathscr{L} \subset \mathscr{H}$ be a Lagrangian with $\mathscr{L} \cap p(\tilde{V})=0$. Then

$$
\operatorname{ker}\left(D \mid L_{1}^{2}\left(E(r) ; P_{+}+\mathscr{L}\right)\right)=\operatorname{ker}(p)=\operatorname{im}\left(H_{A}^{1}(X(r), \Sigma) \rightarrow H_{A}^{1} X(r)\right) .
$$

We will now consider 1-parameter families $A_{t}$ and $\hat{A}_{t}$ of connections related to each other as above for each $t$. In order to insure that the eigenvectors corresponding to our operators vary smoothly in $t$, we shall restrict ourselves to the case where $A_{t}$ and $\widehat{A}_{t}$ are analytic in $t$. We now define these notions more precisely.

Definition 4.4. A path $A_{t}$ of connections on $X \times S U(2)$ is analytic if

$$
d_{A_{t}} u=d_{A_{0}} u+[a(t), u]
$$

for all $u \in \Omega^{*}(X ; s u(2))$, where $a(t)=a_{1} t+a_{2} t^{2}+\cdots$ is an analytic path in $\Omega^{1}(X ; s u(2))$ which converges in the $C^{r}$-norm for all $r>0$.

We will assume that $A_{t}$ and $\widehat{A}_{t}$ are analytic families of connections on $X(\infty) \times S U(2)$ and $\Sigma \times S U(2)$ (respectively) such that for each $t$, $A_{t}$ is related to $\widehat{A}_{t}$ as above. Also assume that either case (I), (II) or (III) (as defined in Proposition 4.3) holds for $A_{t}$ and $\widehat{A}_{t}$ for all $t$ in a neighborhood of 0 (i.e., the case does not jump or change as $t$ passes through 0 ).

It follows that the dimension of $\mathscr{H}(t)=\operatorname{ker} \widehat{D}_{\widehat{A}_{t}}$ is constant, and that, from [9, Theorem VII.3.9], there is an orthonormal basis for $\mathscr{H}(t)$ which varies analytically in $t$ for $t$ near 0 .

By [9, Theorem VII.3.9] we have a family $\left\{\alpha_{i}(t)\right\}$ of orthonormal Hilbert bases of $L^{2}(\widehat{E})$ and corresponding families $\left\{\mu_{i}(t)\right\}$ of eigenvalues such that for each $i, \widehat{D}_{\widehat{A}_{t}} \alpha_{i}(t)=\mu_{i}(t) \alpha_{i}(t)$. By the same theorem, these families are all holomorphic. We now define a family of unitary operators $U_{t}: L^{2}(\widehat{E}) \rightarrow L^{2}(\widehat{E})$ by $U_{t}\left(\alpha_{i}(0)\right)=\alpha_{i}(t)$ for all $i$.

Definition 4.5. The path $\widehat{A}_{t}$ is fine if there exists a family $\left\{\alpha_{i}(t)\right\}$ of analytic paths of eigenvectors so that $U_{t}$ is a bounded holomorphic path of operators on $L^{2}(\widehat{E})$ and also restricts to a bounded holomorphic path of operators on the Sobolev space $L_{1 / 2}^{2}(\widehat{E})$, i.e., if $U_{t}=\sum_{n} U_{n} t^{n}$, where each $U_{n}$ is bounded with respect to both the $L^{2}$ and the $L_{1 / 2}^{2}$ norms, and the series converges with respect to both of these norms. 
Let

$$
\widetilde{V}(t)=\operatorname{ker}\left(D_{A_{t}} \mid \widetilde{L}_{1}^{2}(E(\infty))\right), \quad V(t)=\operatorname{ker}\left(D_{A_{t}} \mid L_{1}^{2}(E(\infty))\right) .
$$

Let $\mathscr{L}(t)$ be a holomorphic path of Lagrangians in $\mathscr{H}(t)$ such that $\mathscr{L}(0) \cap$ $p(\widetilde{V}(0))=0$.

Theorem 4.6. If $\hat{A}_{t}$ is a fine path, then

$$
\mathscr{D}_{t}(r)=D_{A_{t}} \mid L_{1}^{2}\left(E(r) ; P_{+}(t)+\mathscr{L}(t)\right)
$$

is holomorphic in $t$ in the "general sense" (see [9, p. 366]).

The proof of Theorem 4.6 is in Appendix A. (The essence of this proof is to show that the varying domain of $\mathscr{D}_{t}(r)$ can be analytically parametrized by a constant Banach space in such a way that the composition of $\mathscr{D}_{t}(r)$ with the parameterization is an analytic family of operators on this constant Banach space.)

Recall that for each $t, \mathscr{D}_{t}(r)$ is a selfadjoint operator. (The proof of this fact is the same as the proof of Theorem 2.C in [4].) In addition, $\mathscr{D}_{t}(r)$ has compact resolvent, as discussed in $\S 2$. As a result we have the following important corollary.

Corollary 4.7. If $\widehat{A}_{t}$ is a fine path, then the eigenvalues and eigenvectors of $\mathscr{D}_{t}(r)$ are analytic functions of $t$.

This follows from [9, p. 386].

Because of the assumptions on $\mathscr{L}(0)$,

$$
\operatorname{ker}\left(\mathscr{D}_{0}(r)\right)=\operatorname{ker}(p)=\operatorname{im}\left(H_{A_{0}}^{1}(X, \Sigma) \rightarrow H_{A_{0}}^{1} X\right) .
$$

As $t$ varies, the spectrum of $\mathscr{D}_{t}(r)$ changes. The following theorem gives the signs of the derivatives of the eigenvalues of $\mathscr{D}_{t}(r)$ which pass through 0 at time $t=0$ (for large values of $r$ ). The number of such eigenvalues (with multiplicity) is

$$
\operatorname{dim} \operatorname{ker} \mathscr{D}_{0}(r)=\operatorname{dim} \operatorname{im}\left(H_{A_{0}}^{1}(X, \Sigma) \rightarrow H_{A_{0}}^{1} X\right) .
$$

Theorem 4.8. Let $A_{t}$ and $\hat{A}_{t}$ be analytic paths of flat connections on $X(\infty)$ and $\Sigma$ related to each other as above. Assume that $\hat{A}_{t}$ is a fine path. Let $a \in \Omega^{1}(X(\infty) ; s u(2))$ be the derivative of the path $A_{t}$ at $t=0$. In other words, to first order in $t$,

$$
d_{A_{t}}(\omega)=d_{A_{0}} \omega+t[a, \omega]+o\left(t^{2}\right)
$$

for $\omega \in \Omega^{*}(X(\infty) ; s u(2))$. Let $W=\operatorname{im}\left(j^{*}: H_{A_{0}}^{1}(X, \Sigma) \rightarrow H_{A_{0}}^{1} X\right)$. There is a symmetric bilinear form $B: W \times W \rightarrow \mathbb{R}$ defined by:

$$
B\left(j^{*} x, j^{*} y\right)=[x, y] \cdot a \in H^{3}(X, \Sigma ; \mathbb{R})=\mathbb{R} .
$$


The eigenvalues of $B$ are the limits, as $r \rightarrow \infty$, of the derivatives (with respect to $t$ ) of those eigenvalues of $\mathscr{D}_{t}(r)$ which are passing through 0 at $t=0$. In other words, if $B$ is nondegenerate, then "the signature of $B$ gives the contribution at $t=0$ to $S F\left(\mathscr{D}_{t}(r)\right)$."

Remark. In Appendix B we prove that a holomorphic path of connections on a torus in "standard form" is fine. This is sufficient for our applications to Dehn surgery. We have recently shown that Theorem 4.8 continues to hold for any analytic path of flat connections, so that one can drop the assumption that $\hat{A}_{t}$ is fine [11].

This theorem reduces the computation of the "local" contribution to the spectral flow of $\mathscr{D}_{t}(r)$ to a cup product computation in $H_{A_{0}}^{*}(X, \Sigma)$.

Notation. The symbol $[x, y]$ refers to the cup product

$$
H_{A}^{1}(X, \Sigma ; s u(2)) \times H_{A}^{1}(X, \Sigma ; s u(2)) \rightarrow H_{A}^{2}(X, \Sigma ; s u(2))
$$

defined using the Lie bracket on $s u(2)$. Likewise $u \cdot a$ is a cup product, defined using the standard inner product on $s u(2)$, which takes values in cohomology with trivial coefficients in $\mathbb{R}$.

Proof. The symmetry of $B$ follows because the skew-symmetry of the Lie bracket cancels the skew-symmetry of the wedge product of 1-forms. To see that $B\left(j^{*} x, j^{*} y\right)$ depends on $j^{*} x$ instead of on $x$, note that the following diagram commutes by naturality:

$$
\begin{aligned}
H^{1}(X, \sigma) & \otimes H^{1}(X, \Sigma) \backslash[,] \\
j^{*} \otimes \text { id } & \downarrow \\
H^{1}(X) & \otimes H^{1}(X, \Sigma)
\end{aligned}
$$

Fix $r \geq 0$. Let $n=\operatorname{dim} W$. By Corollary 4.7, we know that there is a path of orthonormal sets $\left\{y_{1}(t), \cdots, y_{n}(t)\right\}$ in $L_{1}^{2}\left(E(r) ; P_{+}(t)+\mathscr{L}(t)\right)$ such that for each $t$ near 0 ,

$$
D_{A_{t}}\left(y_{i}(t)\right)=\lambda_{i}(t) y_{i}(t) \text { and } \lambda_{i}(0)=0 \quad \text { for } i=1, \cdots, n \text {. }
$$

Renormalize $\left\{y_{i}(t)\right\}$ so that $\left\{\bar{y}_{i}(0)\right\}$ are of unit size, where $\bar{y}_{i}(0)$ is the canonical extension of $y_{i}(0)$ to $L_{1}^{2}(E(\infty))$. Let $r_{1}<r_{2}<\cdots$ be an unbounded sequence of positive reals. For each $j$, fix $r=r_{j}$ and construct $\left\{y_{1}\left(r_{j}, t\right), \cdots, y_{n}\left(r_{j}, t\right)\right\}$ and $\left\{\lambda_{1}\left(r_{j}, t\right), \cdots, \lambda_{n}\left(r_{j}, t\right)\right\}$ as above. For each $i$, define $\dot{\lambda}_{i}\left(r_{j}\right)=\partial\left(\lambda_{i}\left(r_{j}, t\right)\right) /\left.\partial t\right|_{t=0}$.

To first order, write

$$
y_{i}\left(r_{j}, t\right)=\left(0, c_{i}\left(r_{j}\right)\right)+t\left(e_{i}\left(r_{j}\right), f_{i}\left(r_{j}\right)\right)
$$

for $t$ near 0 , using the fact that $y_{i}\left(r_{j}, 0\right)$ is a pure 1 -form. Note that for each $i$ and $j, \bar{y}_{i}\left(r_{j}, 0\right) \in S^{n}(V)$, the unit $n$-sphere in $V$. By compact- 
ness, replace $\left\{r_{j}\right\}$ by a subsequence such that for each $i=1, \cdots, n$, the sequence $\left\{\bar{y}_{i}\left(r_{j}, 0\right)\right\}$ converges in $S^{n}(V)$. Let $c_{i}^{\infty}=\lim _{j \rightarrow \infty} c_{i}\left(r_{j}\right)$.

Differentiating the expression

$$
D_{A_{t}}\left(y_{i}\left(r_{j}, t\right)\right)=\lambda_{i}\left(r_{j}, t\right) y_{i}\left(r_{j}, t\right)
$$

with respect to $t$ at $t=0$ yields

(*) $D_{A_{0}}\left(e_{i}\left(r_{j}\right), f_{i}\left(r_{j}\right)\right)=\left(0, \dot{\lambda}_{i}\left(r_{j}\right) c_{i}\left(r_{j}\right)\right)-\left(-*\left[a, * c_{i}\left(r_{j}\right)\right], *\left[a, c_{i}\left(r_{j}\right)\right]\right)$.

Let $(0, x) \in V$ be a fixed 1-form. Compute, using $D_{A_{0}}(0, x)=0$,

$$
\begin{aligned}
0 & =\left\langle D_{A_{0}}(0, x),\left(e_{i}\left(r_{j}\right), f_{i}\left(r_{j}\right)\right)\right\rangle_{X\left(r_{j}\right)} \\
& =\left\langle(0, x), D_{A_{0}}\left(e_{i}\left(r_{j}\right), f_{i}\left(r_{j}\right)\right)\right\rangle_{\left(r_{j}\right)} \pm\left\langle(0, x), \sigma\left(e_{i}\left(r_{j}\right), f_{i}\left(r_{j}\right)\right)\right\rangle_{\partial X\left(r_{j}\right)} .
\end{aligned}
$$

By $(*)$, this gives

$$
\begin{aligned}
\left\langle x, \dot{\lambda}_{i}\left(r_{j}\right) c_{i}\left(r_{j}\right)\right\rangle_{X\left(r_{j}\right)}-\left\langle x, *\left[a, c_{i}\left(r_{j}\right)\right]\right\rangle_{X\left(r_{j}\right)} & \\
& = \pm\left\langle(0, x), \sigma\left(e_{i}\left(r_{j}\right), f_{i}\left(r_{j}\right)\right)\right\rangle_{\partial X\left(r_{j}\right)}
\end{aligned}
$$

Lemma 4.9. $\lim _{j \rightarrow \infty}\left\langle(0, x), \sigma\left(e_{i}\left(r_{j}\right), f_{i}\left(r_{j}\right)\right)\right\rangle_{\partial X\left(r_{j}\right)}=0$.

Proof. To prove this lemma, we need to take a closer look at the boundary conditions satisfied by $y_{i}\left(r_{j}, t\right)$.

In the notation of the previous section, recall that

$$
L^{2}(\widehat{E})=H_{\widehat{A}_{t}}^{0}(\Sigma) \oplus H_{\widehat{A}_{t}}^{1}(\Sigma) \oplus H_{\widehat{A}_{t}}^{2}(\Sigma) \oplus \operatorname{span}_{\mu_{t} \neq 0}\left\{\xi_{k}^{+}(t), \xi_{k}^{-}(t), \psi_{k}^{+}(t), \psi_{k}^{-}(t)\right\}
$$

Recall that $\xi_{k}^{+}(t)$ and $\psi_{k}^{+}(t)$ are $\sqrt{\mu_{k}(t)}$-eigenvectors of $\widehat{D}_{\widehat{A}_{t}}$, and $\xi_{k}^{-}(t)$ and $\psi_{k}^{-}(t)$ are $-\sqrt{\mu_{k}(t)}$-eigenvectors of $D_{\widehat{A}_{t}}$. Since $y_{i}\left(r_{j}, t\right) \in \mathscr{L}_{1}^{2}(E(r)$; $\left.P_{+}(t)+\mathscr{H}(t)\right)$, we may write

$$
y_{i}\left(r_{j}, t\right) \mid \partial X\left(r_{j}\right)=h_{i}\left(r_{j}, t\right)+\sum_{k}\left(A_{k}^{i}\left(r_{j}, t\right) \xi_{k}^{+}(t)+B_{k}^{i}\left(r_{j}, t\right) \psi_{k}^{+}(t)\right),
$$

where $h_{i}\left(r_{j}, t\right) \in \mathscr{H}(t)$. We know that $h_{i}\left(r_{j}, 0\right)=0=A_{k}^{i}\left(r_{j}, 0\right)$ for all $i, j, k$, because $\bar{y}_{i}\left(r_{j}, 0\right) \in L_{1}^{2}(E(\infty))$ and hence has no harmonic part on the collar, and the 0 -form part of $y_{i}\left(r_{j}, 0\right)$ is harmonic by Proposition 4.2.

Compute

$$
\left.\left(e_{i}\left(r_{j}\right), f_{i}\left(r_{j}\right)\right)\right|_{\partial X\left(r_{j}\right)}=\left.\frac{d}{d t}\left(\left.y_{i}\left(r_{j}, t\right)\right|_{\partial X\left(r_{j}\right)}\right)\right|_{t=0}=Q_{1}+Q_{2},
$$


where

$$
Q_{1}=\dot{h}_{i}\left(r_{j}, 0\right)+\sum_{k}\left(\dot{A}_{k}^{i}\left(r_{j}, 0\right) \xi_{k}^{+}(0)+\dot{B}_{k}^{i}\left(r_{j}, 0\right) \psi_{k}^{+}(0)\right)
$$

and $Q_{2}=\sum_{k} B_{k}^{i}\left(r_{j}, 0\right) \dot{\psi}_{k}^{+}(0)$.

It is clear that $\left\langle(0, x), \sigma Q_{1}\right\rangle_{\partial X\left(r_{j}\right)}=0$, because $\left.(0, x)\right|_{\partial X\left(r_{j}\right)} \in P_{+}(0)$ and $Q_{1} \in P_{+}(0)+\mathscr{H}(0)$. Hence we need to consider $\lim _{j \rightarrow \infty}\langle(0, x)$, $\left.\sigma Q_{2}\right\rangle_{\partial x\left(r_{j}\right)}$. Because $\left.(0, x)\right|_{\partial X\left(r_{j}\right)}$ decays exponentially in $r_{j}$, it would suffice to show that $Q_{2}$ is bounded in $r_{j}$. In fact, we will show that $Q_{2}$ also decays exponentially in $r_{j}$. First, note that each element of $S^{n}(V)$ has an expression of the form

$$
v=\sum_{k=1}^{\infty} b_{k}(v) e^{-\sqrt{\mu_{k}(0)} s} \psi_{k}^{+}(0)
$$

on the collar $\Sigma \times[0, \infty)$. Since each $\bar{y}_{i}\left(r_{j}, 0\right) \in S^{n}(V)$, we may write $B_{k}^{i}\left(r_{j}, 0\right)=e^{-\sqrt{\mu_{k}} r_{j}} b_{k}(v)$ for $v=\bar{y}_{i}\left(r_{j}, 0\right) \in S^{n}(V)$.

Consider the function $g: S^{n}(V) \rightarrow \mathbb{R}$ defined by

$$
g(v)=\left|\sum_{k} b_{k}(v) \dot{\psi}_{k}^{+}(0)\right| \widehat{\Sigma}
$$

Note that $g$ is the composition of the following maps:

(1) The restriction map

$$
L_{1}^{2}(E(\infty)) \supset S^{n}(V) \rightarrow L_{1 / 2}^{2}(E \mid \Sigma \times\{r\}) \cong L_{1 / 2}^{2}(\widehat{E}) .
$$

(2) The map $\stackrel{\bullet}{U}: L_{1 / 2}^{2}(\widehat{E}) \rightarrow L_{1 / 2}^{2}(\widehat{E})$ given by $\dot{U}\left(\psi_{k}^{+}\right)=\dot{\psi}_{k}^{+}$.

(3) The $L^{2}$-norm map on $L_{1 / 2}^{2}(\widehat{E})$.

The first and third of these are well known to be continuous, and the second is continuous because $\widehat{A}_{t}$ is assumed to be a fine path. It follows that $g$ is continuous.

Because $S^{n}(V)$ is compact $g(v)$ is bounded in $\mathbb{R}$ by a positive number, say $N$. Then it is clear that $\left|Q_{2}\right| \leq e^{-\sqrt{\mu_{1}(0)} r_{j}} N$, where $\mu_{1}(0)$ is the smallest nonzero eigenvalue of $\Delta_{\widehat{A}_{0}}$ on $\Omega^{0}(\sigma ; s u(2))$. This completes the proof of Lemma 4.9. 
By the above lemma, we know that for all $x \in V$,

$$
\begin{aligned}
\lim _{j \rightarrow \infty}\left\langle x, \dot{\lambda}_{i}\left(r_{j}\right) c_{i}\left(r_{j}\right)\right\rangle_{X\left(r_{j}\right)} & =\lim _{j \rightarrow \infty}\left\langle x, *\left[a, c_{i}\left(r_{j}\right)\right]\right\rangle_{X\left(r_{j}\right)}=\left\langle x, *\left[a, c_{i}^{\infty}\right]\right\rangle_{X(\infty)} \\
& =\int_{X(\infty)} \operatorname{tr}\left(x \wedge\left[a, c_{i}^{\infty}\right]\right)=x \cdot\left[a, c_{i}^{\infty}\right] \\
& =\left[x, c_{i}^{\infty}\right] \cdot a=B\left(x, c_{i}^{\infty}\right) .
\end{aligned}
$$

Substituting $c_{i}^{\infty}$ for $x$, we see

$$
B\left(c_{i}^{\infty}, c_{i}^{\infty}\right)=\lim _{j \rightarrow \infty}\left\langle c_{i}^{\infty}, \dot{\lambda}_{i}\left(r_{j}\right) c_{i}\left(r_{j}\right)\right\rangle_{X\left(r_{j}\right)}=\lim _{j \rightarrow \infty} \dot{\lambda}_{i}\left(r_{j}\right),
$$

so this limit exists. Substituting $c_{k}^{\infty}$ for $x, k \neq i$, gives

$$
\begin{aligned}
B\left(c_{k}^{\infty}, c_{i}^{\infty}\right) & =\lim _{j \rightarrow \infty}\left\langle c_{k}^{\infty}, \dot{\lambda}_{i}\left(r_{j}\right) c_{i}\left(r_{j}\right)\right\rangle_{X\left(r_{j}\right)} \\
& =\lim _{j \rightarrow \infty}\left\langle c_{k}\left(r_{j}\right), \dot{\lambda}_{i}\left(r_{j}\right) c_{i}\left(r_{j}\right)\right\rangle_{X\left(r_{j}\right)}=0 .
\end{aligned}
$$

It follows that the form $B$ is diagonal with respect to the basis $\left\{c_{i}^{\infty}\right\}_{i}$ with eigenvalues $\left\{\lim _{j \rightarrow \infty} \dot{\lambda}_{i}\left(r_{j}\right)\right\}_{i}$. Hence for large enough values of $j$, the signs of $\left\{\dot{\lambda}_{i}\left(r_{j}\right)\right\}_{i}$ are precisely the signs of the eigenvalues of the form $B$.

\section{Spectral flow along arcs of flat connections on a closed 3-manifold}

In this section we prove a theorem analogous to Theorem 4.8 which holds in the case of a closed 3-manifold $M$. This theorem gives the spectral flow of a path of operators $D_{A_{t}}$ on $M$, where $A_{t}$ is an analytic path of flat connections on $M$. The analysis involved is considerably easier since there are no boundary conditions to worry about.

Throughout this section, $M$ will denote a closed orientable 3-manifold with a fixed Riemannian metric. Let $A_{t} \in \Omega^{1}(M ; s u(2))$ be an analytic path of flat connections, defined for $t \in(-\varepsilon, \varepsilon)$. Define the vector bundle $E \rightarrow M$ and the operator $D_{A_{t}}$ just as in $\S 3$. Note that the domain of $D_{A_{t}}$ is constant as $t$ varies; it is the image of the $L_{1}^{2}$-sections in $L^{2}$. It follows from [9, Theorem VII.3.9] that the (orthornormal) eigenvectors $\left\{\alpha_{i}(t)\right\}$ and corresponding eigenvalues $\left\{\lambda_{i}(t)\right\}$ of $D_{A_{t}}$ are analytic functions of $t$.

Let $W=\operatorname{ker} D_{A_{0}} \cong H_{A_{0}}^{0}(M) \oplus H_{A_{0}}^{1}(M)$, where $H_{A}^{*}(M)$ denotes the DeRham cohomology of $M$ with coefficients in $s u(2)$ using the flat connection $A$. If $n=\operatorname{dim} W$, then there are exactly $n$ eigenvalues (counted 
with multiplicity) of $D_{A_{t}}$ passing through 0 at $t=0$. After reordering, assume $\lambda_{1}(0)=\lambda_{2}(0)=\cdots=\lambda_{n}(0)=0$, so $\left\{\alpha_{i}(0)\right\}_{i=1}^{n}$ is an orthonormal basis for $W$. The following theorem gives the signs of the derivatives $\dot{\lambda}_{i}=d /\left.d t\right|_{t=0} \lambda_{i}(t)$ for $i=1, \cdots, n$.

Theorem 5.1. Let $a \in \Omega^{1}(M ; s u(2))$ be the derivative $d /\left.d t\right|_{t=0} A_{t}$. Define a symmetric bilinear form $G$ on $W$ as follows: $G(x, y)=\langle x, A y\rangle$ where $A: W \rightarrow \Gamma(E)$ is defined by

$$
A\left(y_{0}, y_{1}\right)=\left(-*\left[* a, y_{1}\right],\left[a, y_{0}\right]+*\left[a, y_{1}\right]\right) .
$$

Then the eigenvalues of $G$ (as a set with multiplicity) are $\left\{\dot{\lambda}_{1}, \cdots, \dot{\lambda}_{n}\right\}$.

Note. If we choose the form $a$ to be harmonic (e.g., by applying a path of gauge transformations to $\left.\left\{A_{t}\right\}\right)$, then we may write $G(x, y)$ in terms of cup products as we did in Theorem 4.8:

$$
G\left(\left(x_{0}, x_{1}\right),\left(y_{0}, y_{1}\right)\right)=\left(\left[x_{0}, y_{1}\right]-\left[x_{1}, y_{0}\right]\right) \cdot * a+\left[x_{1}, y_{1}\right] \cdot a .
$$

Because $a$ is harmonic, $* a \in H^{2}(M ; s u(2))$ so this formula can be interpreted in terms of the two types of cup products discussed following Theorem 4.8.

Proof. During this proof, we will always assume $i \in\{1, \cdots, n\}$. Let $\dot{\alpha}_{i}=d /\left.d t\right|_{t=0} \alpha_{i}(t)$. Let $A: \Gamma(E) \rightarrow \Gamma(E)$ be defined by $A u=$ $d /\left.d t\right|_{t=0} D_{A_{t}} u$. Explicitly, $A$ is given by

$$
A\left(u_{0}, u_{1}\right)=\left(-*\left[a, * u_{0}\right],\left[a, u_{0}\right]+*\left[a, u_{1}\right]\right) .
$$

(While comparing this formula with the definition of $A$ given in the statement of the theorem, note that $\left[* a, u_{0}\right]=\left[a, * u_{0}\right]$.) Differentiating the equation $D_{A_{t}} \alpha_{i}(t)=\lambda_{i}(t) \alpha_{i}(t)$ at $t=0$ gives

$$
D_{A_{0}} \dot{\alpha}_{i}=\dot{\lambda}_{i} \alpha_{i}(0)-A \alpha_{i}(0) .
$$

Let $x \in W$, so $D_{A_{0}} x=0$. Then by selfadjointness,

$$
0=\left\langle D_{A_{0}} x, \dot{\alpha}_{i}\right\rangle=\left\langle x, D_{A_{0}} \dot{\alpha}_{i}\right\rangle=\left\langle x, \dot{\lambda}_{i} \alpha_{i}(0)-A \alpha_{i}(0)\right\rangle .
$$

Hence for all $i \in\{1, \cdots, n\}$,

$$
\dot{\lambda}_{i}\left\langle x, \alpha_{i}(0)\right\rangle=\left\langle x, A \alpha_{i}(0)\right\rangle .
$$

Because this equation holds on the basis $\left\{\alpha_{i}(0)\right\}$ of $W$, the theorem follows. 


\section{Dehn fillings}

In this section we return to our overall project of calculating the spectral flow of the path $D_{A_{t}}$ of operators along the path $A_{t}$ of connections on a manifold $M=X \cup_{\Sigma} Y$ where, for each $t, A_{t}$ is assumed to be flat on $X$. Recall that according to Theorem 2.1, we have the formula

$$
S F\left(D_{A_{t}}\right)=S F\left(D_{X}(t)\right)+S F\left(D_{Y}(t)\right)+\gamma\left(L_{X}(t), L_{Y}(t)\right) .
$$

In $\S 4$, Theorem 4.8, we gave a method of calculating the first term, $S F\left(D_{X}(t)\right)$. We needed several assumptions to make this computation; an important one was that $L_{X}(t)$ should be chosen to be transverse to the Lagrangian $p(\tilde{V}(t))$ at each time $t$.

We will now turn to the computation of the sum of the other two terms in Theorem 2.1, namely

$$
S F\left(D_{Y}(t)\right)+\gamma\left(L_{X}(t), L_{Y}(t)\right) .
$$

Though much of this section could be adapted to apply to an arbitrary 3-manifold $Y$ with boundary, we will restrict ourselves in this section to the case in which $Y=S^{1} \times D^{2}$. We make this restriction because it greatly simplifies the technicalities, and also because this case is adequate for the applications which we have in mind (see $\S 7$ ). We will at times make remarks indicating how one might generalize to other choices of $Y$.

We will observe that the sum of these two terms is independent of any information about $X$; in fact it depends only on the path $A_{t} \mid \Sigma$ of flat connections on $\Sigma$ and on the path $L_{X}(t)$ of Lagrangians. In an important sense we will see that it depends only on the homotopy classes of these paths (see Lemma 6.2).

By considering certain "test cases" we will determine precisely the dependence on the relevant homotopy classes. This dependence was essentially computed by Yoshida in [20]. However our proof is somewhat more direct (it does not required adding handles to obtain a higher genus surface), and Yoshida never states the precise form of this theorem which we will need for our applications. Hence, in this section we will present our own statement and proof of this theorem (Theorem 6.4).

In what follows we (continue to) identify $S U(2)$ with the unit quaternions.

The space of $S U(2)$-conjugacy classes of representations of the fundamental group of a torus, $R(T)$, is a singular real algebraic variety homeomorphic to $S^{2}$ with four singular points corresponding to central representations. Let $Y=D^{2} \times S^{1}$ be a solid torus and let $T=\partial Y$. 
Let $\mu, \lambda \in \pi_{1}(T)$ be a pair of generators so that $\mu$ bounds a disk in $Y$. The map $\mathbb{R}^{2} \rightarrow R(T)$ sending a pair $(\alpha, \beta)$ to the representation $\mu \mapsto e^{2 \pi i \alpha}, \lambda \mapsto e^{2 \pi i \beta}$ is a branched cover, with branch points the halfinteger lattice which map to the central representations. This branched cover also determines a 2-parameter family of flat connections on the $T \times S U(2)$, namely $(\alpha, \beta)$ corresponds to the connection with 1 -form $i \alpha d x+i \beta d y$. Here $x$ and $y$ are coordinates on the torus such that the coordinate axes correspond to $\mu$ and $\lambda$. (We identify connections on $T \times S U(2)$ with 1 -forms by taking the trivial connection to 0 .) The conjugacy classes of representations of $\pi_{1} T$ in $S U(2)$ form a space homeomorphic to the gauge-equivalence classes of flat connections on $T$, but using this branched cover we have a family of connections, not just gaugeequivalence classes.

Now $\widehat{A}=i \alpha d x+i \beta d y$ extends to a flat connection on $Y$ if and only if $\alpha$ is an integer. A precise formula for an extension can be found in [10, p. 352]. Let $\mathscr{F}(T)$ denote the flat connections on $T$, and let $\mathscr{A}^{f}(Y)$ denote those connections on $Y$ which, when restricted to the collar $T \times[-1,0]$ are a product of a flat connection on $T$ and the trivial connection on $[-1,0]$. The restriction map $r: \mathscr{A}^{f}(Y) \rightarrow \mathscr{F}(T)$ is a trivial fibration with contractible fiber

$$
F=\left\{\omega \in \Omega_{Y}^{1} \mid \omega=0 \text { on } T \times[-1,0]\right\} .
$$

(A cross section can be constructed by using a slightly larger collar to cut off the product of a flat connection with the trivial connection.)

Restrict the fibration $r$ to $\left\{i \alpha d x+i \beta d y \mid(\alpha, \beta) \in \mathbb{R}^{2}\right\} \cong \mathbb{R}^{2}$. Extending the connections with $\alpha \in \mathbb{Z}$ flatly over $Y$ gives a (continuous) cross section $\chi$ of $r$ over $\mathbb{Z} \times \mathbb{R} \subset \mathbb{R}^{2}$, the union of vertical lines. Since the fiber is contractible, $\chi$ extends to a section over all of $\mathbb{R}^{2}$, although the extensions will be flat on $Y$ only over the vertical lines. We will need to avoid connections whose restrictions to $T$ give central representations. Therefore we define

$$
R=\chi\left(\mathbb{R}^{2}-\left(\frac{1}{2} \mathbb{Z}\right)^{2}\right) \subset \mathscr{A}^{f}(Y)
$$

So $R$ is a family of connections on $Y=D^{2} \times S^{1}$ parameterized by the punctured plane $\mathbb{R}^{2}-\left(\frac{1}{2} \mathbb{Z}\right)^{2}$. Write $\hat{A}$ for the restriction of $A \in R$ to the boundary torus. We summarize the properties of $R$ :

1. If $A \in R$, then on the collar $A=\theta \times \hat{A}$, the product of the trivial connection in the collar direction with the flat connection on the torus $\hat{A}=i \alpha d x+i \beta d y$. (We say $A$ is an standard form on the collar.) 
2. If $A \in R$ and $\widehat{A}=i \alpha d x+i \beta d y$, then at least one of $\alpha$ and $\beta$ is not in $\frac{1}{2} \mathbb{Z}$.

3. If $A \in R$, then $A$ is flat if and only if $\widehat{A}=i \alpha d x+i \beta d y$ has $\alpha \in \mathbb{Z}$.

Write

$$
Q=\{A \in R \mid \hat{A}=i \alpha d x+i \beta d y, \alpha \in \mathbb{Z}\} \subset R .
$$

So elements of $Q$ are flat connections on $Y$.

We assume that the Riemannian metric on the torus is chosen so that $\{d x, d y\}$ form an orthonormal basis, and the metric on the solid torus is a product metric near the boundary. The forms $\omega \in \Omega^{p} \otimes s u(2)$ which are harmonic with respect to $\widehat{A}=i \alpha d x+i \beta d y$ (i.e., $d_{\widehat{A}} \omega=0=d_{\widehat{A}}^{*} \omega$ ) are easy to compute and in fact are independent of $\alpha$ and $\beta$ as long as these are not both in $\frac{1}{2} \mathbb{Z}$ : the harmonic 0 -forms are just $\{i a \mid a \in \mathbb{R}\} \cong \mathbb{R}$, the harmonic 1-forms are $\{i a d x+i b d y \mid a, b \in \mathbb{R}\} \cong \mathbb{R}^{2}$, and the harmonic 2-forms are $\{$ ia $d x d y \mid a \in \mathbb{R}\} \cong \mathbb{R}$.

Over $R$ we have the (trivial) symplectic 4-dimensional vector bundle of harmonic forms on the torus, $\mathscr{H}=\mathscr{H}^{0} \oplus \mathscr{H}^{1} \oplus \mathscr{H}^{2}$ where

$$
\begin{gathered}
\mathscr{C}^{0}=R \times\{a i \mid a \in \mathbb{R}\}, \\
\mathscr{H}^{1}=R \times\{\text { aid } x+b i d y \mid a, b \in \mathbb{R}\},
\end{gathered}
$$

and

$$
\mathscr{C}^{2}=R \times\{\text { ai } d x d y \mid a \in \mathbb{R}\} .
$$

The symplectic structure is given by $\sigma\left(\alpha^{0}, \alpha^{1} \alpha^{2}\right)=\left(-* \alpha^{2}, * \alpha^{1}, * \alpha^{0}\right)$, as described in $\S 2$.

Remark. Given any continuous family of connections on any surface so that the dimension of the kernels of the family $\widehat{D}_{\widehat{A}}$ is constant, one obtains a corresponding bundle $\mathscr{H}$ over the parameter space whose fiber over $A$ is $\operatorname{ker} \widehat{D}_{\widehat{A}}$. This follows from the fact that the family $\widehat{D}_{\widehat{A}}$ forms a continuous family of closed operators in the graph topology and Theorem IV.3.16 of [9]. For our connections in standard form over the torus this bundle is canonically trivial.

We let $\mathscr{L} \subset \mathscr{H}$ be the (trivial) Lagrangian subbundle whose fiber at each point of $R$ is

$$
\mathscr{L}_{a}=0 \oplus \mathbb{R} i d x \oplus \mathbb{R} i d x d y .
$$

The important observation for our purposes is that $\sigma(\mathscr{L})$ equals $p(\tilde{V})$ along $Q$, and, in particular

1. $p(\widetilde{V})$ forms a smooth Lagrangian subbundle of $\mathscr{H}$ along $Q$, and

2. $\mathscr{L}$ is transverse to $p(\widetilde{V})$ along $Q$. 
To see this, first notice that a choice of meridian and longitude gives a canonical identification of $H^{1}(\partial Y$; ad $\rho)$ with $\mathbb{R}^{2}$ if $\rho$ is a noncentral representation. (For example this is isomorphic to $\mathscr{H}^{1}$.) Then Proposition 4.3(III), together with the each computation that the image $\operatorname{im}\left(H^{1}(Y ; \operatorname{ad} \rho)\right) \rightarrow H^{1}(\partial Y ; \operatorname{ad} \rho) \cong \mathbb{R}^{2}$ is constant as $\rho$ varies in the noncentral representations of $\pi_{1} Y$, shows that $p(\widetilde{V}) \subset \mathscr{H}$ is constant and in fact equals the restriction of $\sigma(\mathscr{L})$ to $Q$, namely $\mathbb{R} i \oplus \mathbb{R} i d y \oplus 0$.

This choice of $\mathscr{L}$ is not canonical, and one might try other choices. A more natural choice is to take $\mathscr{L}_{A}=\sigma\left(p\left(\widetilde{V}_{A}\right)\right)$ for all $A \in R$; this is the choice taken in [19]. Unfortunately, this is not in general a continuous subbundle. We will give an example, at the end of this section, of a smooth path of flat connections on the trefoil knot complement so that $p(\widetilde{V})$ is a discontinuous subbundle of $\mathscr{H}$. Our choice is the one which makes the computation easiest. (In [19], Yoshida proved that the Riemannian metric and a path of connections could be perturbed so that $p(\widetilde{V})$ is continuous along the path. His proof requires that the boundary surface have genus greater than 1.)

For $A \in R$, let

$$
\mathscr{D}_{A}(\mathscr{L})=D_{A} \mid L_{1}^{2}\left(E ; P_{+}(\widehat{A})+\mathscr{L}_{A}\right),
$$

where $D_{A}$ is the operator on $E=\left(\Omega^{0} \oplus \Omega^{1}\right)(Y ; s u(2))$ constructed in $\S 2$, and $P_{+}(\widehat{A})$ refers to a span of the positive eigenvalues of the operator $\widehat{D}_{\widehat{A}}$.

Notice that the equation $D_{A} \phi=0$ has no $L^{2}$ solutions if $A \in Q$. The reason is that since $A$ is flat, the $L^{2}$-solutions equal the image of the relative cohomology in the absolute cohomology [1]. But $H^{0}\left(Y, T ;\right.$ ad $\left.\rho_{A}\right)=$ 0 and $H^{1}\left(Y, T ;\right.$ ad $\left.\rho_{A}\right)=0$. It follows that if $A \in Q$, then $\mathscr{D}_{A}(\mathscr{L})$ has no kernel. (There are no extended $L^{2}$ solutions because of the assumption that $\mathscr{L}$ is transverse to $p(\widetilde{V}))$.

Let $\mathrm{Lag} \rightarrow R$ denote the fiber bundle whose fiber over $A$ is the space of Lagrangian subspaces of $\mathscr{H}_{A}$.

Lemma 6.1. The map Lag $\rightarrow$ \{selfadjoint, closed operators on $\left.L^{2}(E)\right\}$ which takes a Lagrangian $L \subset \mathscr{H}_{A}$ to the operator $\mathscr{D}_{A}\left(P_{+}(\widehat{A})+L\right)$ is continuous in the graph topology on closed operators.

The proof of this lemma is contained in Appendix B, and works for any manifold whose boundary is a union of tori, not just $S^{1} \times D^{2}$. (In a more recent paper [11] we show that Lemma 6.1 holds for any surface. In particular, there are analogues of Theorem 6.4 for any 3-manifold with boundary.) 
Let $C^{0}(R, Q)$ denote the space of all continuous paths $I \rightarrow R$ with endpoints in $Q$. It follows from Lemma 6.1 that given any path $q \in$ $C^{0}(R, Q)$ the spectrum of the operator $\mathscr{D}_{q(t)}(\mathscr{L})$ varies continuously in $t\left[9\right.$, pp. 107, 291]. Thus we can think of the spectral flow of $\mathscr{D}_{q(t)}(\mathscr{L})$ as a integer-valued function on $C^{0}(R, Q)$.

Lemma 6.2. The function $S F(\mathscr{D}(\mathscr{L})): C^{0}(R, Q) \rightarrow \mathbb{Z}$ is a homotopy invariant and is additive with respect to composition of paths. In particular, there exists a cohomology class $\chi \in H^{1}(R, Q ; \mathbb{Z})$ so that $\operatorname{SF}\left(\mathscr{D}_{q}(\mathscr{L})\right)$ $=\chi([q])$.

Proof. Notice that by hypothesis on $\mathscr{L}$, the operator $\mathscr{D}_{A}(\mathscr{L})$ has no kernel if $A$ lies in $Q$. In particular the spectral flow is zero for paths lying entirely in $Q$. Suppose that $q$ and $q^{\prime}$ are homotopic paths in $R$ via a homotopy keeping their endpoints in $Q$. Using the homotopy one sees that the spectral flow of $\mathscr{D}(\mathscr{L})$ ) is the same along $q$ and $q^{\prime}$. Now $S F(\mathscr{D}(\mathscr{L}))$ is clearly additive with respect to unions of paths. These facts guarantee that there exists a class $\chi$ with the stated properties. q.e.d.

Suppose we wish to apply Theorem 2.1 under the following conditions. $A_{t}$ is a path of connections on $X \cup_{T^{2}} Y$ such that $A_{t} \mid X$ is flat for all $t$, and $A_{0}, A_{1}$ are both flat on $X \cup Y$. We will also assume that on the collar $T^{2} \times I$, we have $A_{t}=\pi^{*}\left(\widehat{A}_{t}\right)$, where $\hat{A}_{t}$ is in standard form and has noncentral holonomy for all $t \in[0,1]$. In addition, we assume that $A_{t} \mid X$ is irreducible for all $t$. Because $\hat{A}_{t}$ is in standard form for each $t$, it may be thought of as a path in $R$. We will denote this path by $q: I \rightarrow R$. We will make the following assumptions on $q$ (which are actually implied assumptions on $A_{t}$ ):

(1) $q: I \rightarrow R$ is an immersion.

(2) $I \cap q^{-1}(Q)=\{0,1\}$.

(3) $d q / d t$ is transverse to $Q$ at $q(0)$ and $q(1)$.

Definition. We define $\operatorname{Imm}(R, Q)$ to be the set of all smooth paths $q: I \rightarrow R$ satisfying (1), (2), and (3) above.

In order to calculate $S F_{X}\left(D_{A_{t}}\right)$ using Theorem 4.8 we will need to choose a path $\mathscr{K}_{t}$ of Lagrangians in $\mathscr{H}$ with the property that for all $t \in[0,1], \mathscr{K}_{t} \cap p\left(\widetilde{V}_{X}(t)\right)=0$. Thus the following lemma will be helpful.

Lemma 6.3. Assume that $A_{t}$ and $q$ satisfy all of the above conditions.

Then

$$
p\left(\widetilde{V}_{X}\left(t_{0}\right)\right)=0 \oplus \operatorname{Span}\left(\left.\frac{d q}{d t}\right|_{t=t_{0}}\right) \oplus \operatorname{Span}(i d x d y) .
$$


Proof. By Proposition 4.3, we know that

$$
p\left(\widetilde{V}_{X}\left(t_{0}\right)\right)=0 \oplus \operatorname{im}\left(j^{*}: H_{A_{t_{0}}}^{1}(X) \rightarrow H_{\widehat{A}_{t_{0}}}^{1}\left(T^{2}\right)\right) \oplus \operatorname{span}(i d x d y) .
$$

So we need to show that $\operatorname{im}\left(j^{*}\right)=\operatorname{span}\left(d q /\left.d t\right|_{t=t_{0}}\right)$. Since $A_{t} \mid X$ is flat for all $t$,

$$
\left.\frac{d A_{t}}{d t}\right|_{t=t_{0}} \in H_{A_{t_{0}}}^{1}(X)
$$

Tracing through the definitions, we see that

$$
j^{*}\left(\left.\frac{d A_{t}}{d t}\right|_{t=t_{0}}\right)=\left.\frac{d \widehat{A}_{t}}{d t}\right|_{t=t_{0}}=\left.\frac{d q}{d t}\right|_{t=t_{0}} .
$$

Because $q$ is an immersion, $d q / d t \neq 0 \in H_{\widehat{A}_{t_{0}}}^{1}\left(T^{2}\right)$. By Poincaré duality, $\operatorname{im}\left(j^{*}\right)$ is 1 -dimensional, so $\operatorname{im}\left(j^{*}\right)=\operatorname{span}(d q / d t)$.

We then see that it is easy to choose $\mathscr{K}_{t}$ transverse to $p\left(\widetilde{V}_{X}(t)\right)$ by setting

$$
\mathscr{K}_{t}=\sigma\left(0 \oplus \operatorname{span}\left(\frac{d q}{d t}\right) \oplus \operatorname{span}(i d x d y)\right)=\operatorname{span}(i) \oplus \operatorname{span}\left(* \frac{d q}{d t}\right) \oplus 0 .
$$

Note that with this shoice of $\mathscr{K}_{t}$ it follows that $\mathscr{K}_{t}$ is transverse to $\mathscr{L}_{i}$ for $i=0,1$ because of the transversality condition at $q(0)$ and $q(1)$. Thus to each path $q \in \operatorname{Imm}(R, Q)$, we have assigned a path of Lagrangians $\mathscr{K}_{t} \subset \mathscr{H}$. This path is well-suited for the application of Theorems 2.1 and 4.8 in the cases where $q$ arises from a path $A_{t}$ of flat connections on $X$.

Definition. If $q \in \operatorname{Imm}(R, Q)$, define the writhe of $q$ to be the Maslov index of $\operatorname{span}(d q / d t)$ and the constant path of Lagrangians $(i d y)$ in the space $\mathscr{H}^{1}=\operatorname{span}(i d x, i d y)$. This is simply the intersection number rel boundary of the two paths $t \mapsto(t, \operatorname{span}(d q / d t))$ and $t \mapsto(t, \operatorname{span}(i d y))$ in $I \times \mathbb{R} P^{1}$.

With these choices, we have

Theorem 6.4. Given a smooth immersed patk $q \in \operatorname{Imm}(R, Q)$,

(I) the integer $\gamma(\mathscr{K}, \mathscr{L})$ is the writhe of $q$.

(II) The mod 8 reduction of the cohomology class $\chi$ determined by $\operatorname{SF}(\mathscr{D}(\mathscr{L}))$ in Lemma 6.2 is given by intersecting a path $q$ with the cycle represented in Figure 1.

\section{Remarks.}

1. Given two gauge-equivalence classes of $S U(2)$ connections on a closed 3-manifold $M$, their spectral flow is well-defined only modulo 8. The usual proof of this fact is to lift a loop in $\mathscr{B}=\mathscr{A} / \mathscr{G}$ 


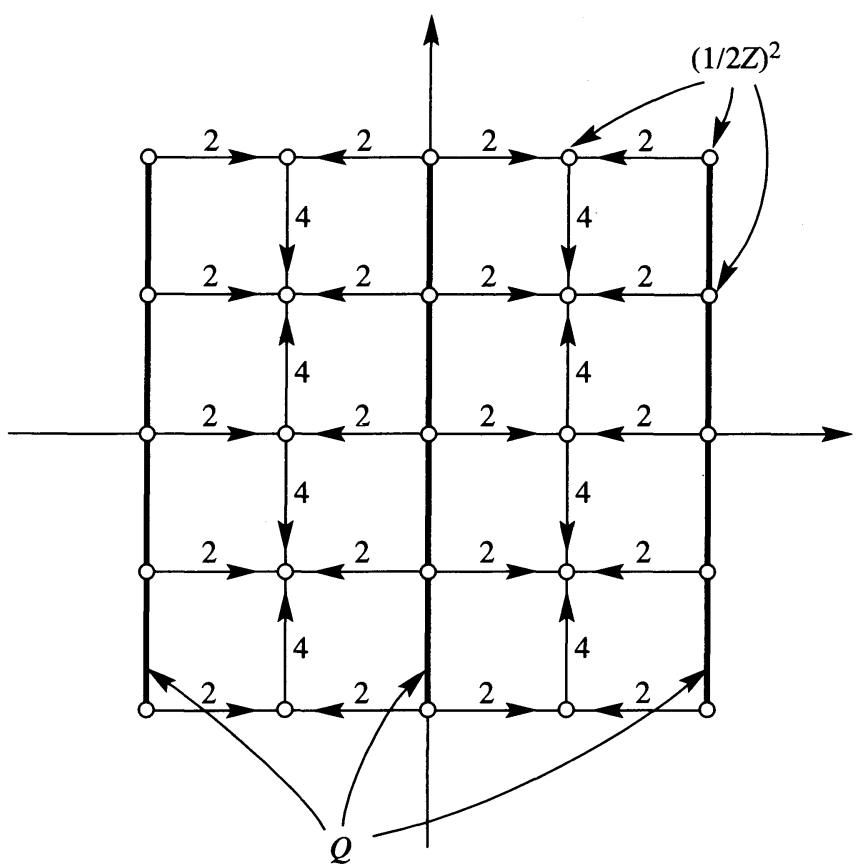

FIGURE 1

to a path in $\mathscr{A}$. This path then defines a connection on an $S U(2)$ bundle over $M \times S^{1}$, and the spectral flow on $M$ is identified with the index of the self-duality operator on $M \times S^{1}$. This index in turn is computed by the Atiyah-Singer index theorem to be $8 k-3\left(1-b_{1}\left(M \times S^{1}\right)+b_{2}^{+}\left(M \times S^{1}\right)\right)$ which is equal to $8 k$ since the signature of $M \times S^{1}$ is zero.

2. Theorem 6.4 generalizes a result of Yoshida [20] which considers only the case of smooth paths of flat connections on $X$, which in this context corresponds to a situation in which the first term $S F_{X}\left(\mathscr{D}_{t}\right)$ in Theorem 1 vanishes.

Proof. The proof of (I) follows from the observation that if $L_{t}, K_{t}$ are two paths of Lagrangians in a symplectic vector space $H$, and we give $\mathbb{R} \oplus H \oplus \mathbb{R}$ the obvious symplectic structure, then

$$
\gamma\left(L_{t}, K_{t}\right)=\gamma\left(\mathbb{R} \oplus L_{t} \oplus 0,0 \oplus K_{t} \oplus \mathbb{R}\right) .
$$

To prove (II), it suffices to try some test cases since any cohomology class in $H^{1}(R, Q ; \mathbb{Z})$ is the intersection with some cycle of the form described for some choice of integers; it suffices to identify these integers. The ar- 


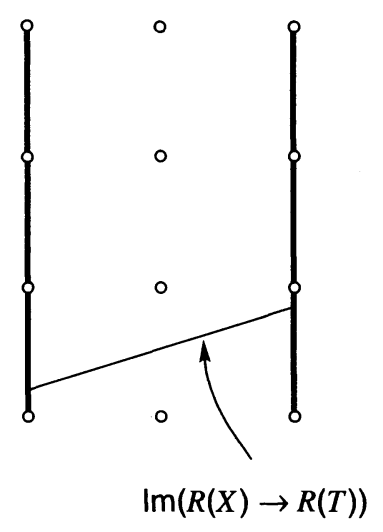

FIGURE 2

gument is basically similar to Yoshida's: the computations of Fintushel and Stern [6] for surgeries on the trefoil knot give us sufficiently many test cases. For one example, consider +1 surgery on the right-handed trefoil knot which is the Poincare homology sphere. Write this as $X \cup_{T} Y$, where $X$ is the trefoil complement, and $Y=D^{2} \times S^{1}$ is the solid torus glued back in. The space of non-abelian representations of $X$ is a smooth arc [10]. This maps to $R$ as shown in Figure 2.

In this figure one sees that there are two representations which send the meridian to 1 , i.e., which extend to representations of $\pi_{1} M$. This path intersects a single vertical line. The spectral flow on $M$ between these two representations has been computed in [6] to be $4 \bmod 8$. On the other hand, since the path between the two representations is a piece of a smooth, 1-dimensional real algebraic variety, the Zariski tangent space $H^{1}\left(X ; \operatorname{ad} \rho_{t}\right)$ is 1 -dimensional and maps injectively to $H^{1}\left(\partial X ; \operatorname{ad} \rho_{t}\right)$. Thus the image $X^{1}\left(X, \partial X ; \operatorname{ad} \rho_{t}\right) \rightarrow H^{1}\left(X ; \operatorname{ad} \rho_{t}\right)$ is zero for all $t$ along this path. Therefore, the operators $\mathscr{D}_{A_{t}}(X)$ have no kernel along the entire arc, and the spectral flow must equal zero. Thus the sum of the other two terms in Theorem 2.1 is equal to $4 \bmod 8$ for this path.

Other examples (e.g., $+1 / 2$ surgery on the trefoil) give paths which intersect the horizontal lines. These paths can be used in the same way to finish the proof. q.e.d.

We finish this section with the promised example showing that the spaces $p(\widetilde{V})$ need not form a smooth or even continuous subbundle of $\mathscr{H}$, even when the path of connections is smooth and the bundle $\mathscr{H}$ is smooth. As remarked above, discontinuities, if there are any, arise at points where there are $L^{2}$ solutions to $D_{A} \phi=0$. 
Let $X$ be the complement of the trefoil knot. Then $\pi_{1} X=\langle x, y| x^{2}=$ $\left.y^{3}\right\rangle$. A smooth path of representations is given by

$$
\rho_{t}(x)=(\cos t) i+(\sin t) j, \quad \rho_{t}(y)=\cos \frac{\pi}{3}+\sin \frac{\pi}{3} i, \quad t \in(-.1, .1) .
$$

This path is abelian for $t=0$, and nonabelian for $t \neq 0$. The meridian is given in these generators by $\mu=y^{2} x^{-1}$. The real part of $\rho_{t}(\mu)$ is equal to $(\sqrt{3} / 2) \cos t$, and since this is not equal to \pm 1 in this interval, it follows that the restriction of $\rho_{t}$ to the boundary is non-central for all $t \in(-.1, .1)$. According to Proposition 4, when $t \neq 0$,

$$
p(\tilde{V})=\operatorname{im}\left(H^{1}\left(X ; \operatorname{ad} \rho_{t}\right) \rightarrow H^{1}\left(\partial X ; \operatorname{ad} \rho_{t}\right)\right) \oplus H^{2}\left(\partial X ; \operatorname{ad} \rho_{t}\right) .
$$

and when $t=0$,

$$
p(\widetilde{V})=H^{0}\left(\partial X ; \operatorname{ad} \rho_{0}\right) \oplus \operatorname{im}\left(H^{1}\left(X ; \operatorname{ad} \rho_{0}\right) \rightarrow H^{1}\left(\partial X ; \operatorname{ad} \rho_{0}\right)\right) .
$$

Since $H^{0}\left(\partial X ; \operatorname{ad} \rho_{t}\right) \cong \mathbb{R} \cong H^{2}\left(\partial X ; \operatorname{ad} \rho_{t}\right)$ for all $t, p(\widetilde{V})$ does not form a continuous subbundle.

Actually, the situation in this example is much worse: the subspaces $\operatorname{im}\left(H^{1}\left(X ; \operatorname{ad} \rho_{t}\right) \rightarrow H^{1}\left(\partial X ; \operatorname{ad} \rho_{t}\right)\right)$ do not form a continuous family even though $\mu$ and $\lambda$ give a canonical identification of $\left.H^{1}\left(\partial X ; \operatorname{ad} \rho_{t}\right)\right)$ with $\mathbb{R}^{2}$ for all $t$. So even if one attempts to "mod out" the 0 - and 2-dimensional part of $H^{*}(\partial X)$ the corresponding family of Lagrangians still does not form a continuous family. We leave the proof of this fact to the interested reader. The basic idea is that when $t \neq 0$ the image $\operatorname{im}\left(H^{1}\left(X ; \operatorname{ad} \rho_{t}\right) \rightarrow H^{1}\left(\partial X ;\right.\right.$ ad $\left.\left.\rho_{t}\right)\right)$ is tangent to the image of the irreducible representations in $R(\partial X)$, but at $t=0$ this image is tangent to the image of the arc of abelian representations $R(\partial X)$.

\section{Application to torus bundles}

We will apply the results of the previous sections to compute the spectral flow mod 4 between two representations of the fundamental group of a 3-manifold $M$ which fibers over the circle with fiber a torus. To compute the spectral flow between two nonabelian representations, we will use Theorems 4.8 and 6.4. The basic idea is to find a knot $\gamma$ in $M$ so that the complement of $\gamma$ admits a path of representations joining the two given representations. The results of $\S 4$ will be used to evaluate the spectral flow on $M-\gamma$, and the results of $\S 6$ will be used to compute the other two terms in Theorem 2.1. Later in this section we will use Theorem 5.1 to compute the spectral flow between two abelian representations. 
We first show that if $\rho_{0}$ and $\rho_{1}$ are nonabelian representations of a torus bundle over the circle, $M$, then there exist a knot $\gamma$ in $M$ and a "straight line" path of representations of $\pi(M-\gamma)$ from $\rho_{0}$ to $\rho_{1}$. The construction works also for an arbitrary surface bundle over $S^{1}$, and so we will consider this more general case. This has independent interest; in particular the result of [10] can be applied to this path to give yet another method for computing the Chern-Simons invariants of special representations of surface bundles (see [2]).

Let $F$ be a closed, oriented surface, and let $m: F \rightarrow F$ be a homeomorphism. Let $M$ be the mapping torus

$$
M=F \times I / \sim
$$

where $(x, 0) \sim(m(x), 1)$. So $M$ is an $F$ bundle over $S^{1}$. The fundamental group of $M$ is an HNN extension:

$$
\left.\pi_{1} M=\left\langle\pi_{1} F, \tau\right| \tau z \tau^{-1}=m(z) \text { for each } z \in \pi_{1} F\right\rangle .
$$

Let $B: H_{1}(F ; \mathbb{Z}) \rightarrow H_{1}(F ; \mathbb{Z})$ be the map induced by $m$ on homology. Fix a generating set $x_{1}, \cdots, x_{2 g}$ for $\pi_{1} F$ forming a symplectic basis for $H_{1}(F ; \mathbb{Z})$, and use this basis to identify $B$ with a $2 g \times 2 g$ matrix (acting on the left).

In [10] we called a nonabelian representation $\rho: \pi_{1} M \rightarrow S U(2)$ a special representation if the restriction of $\rho$ to a fiber is abelian. One checks that given a vector $\phi=\left(\phi_{1}, \cdots, \phi_{2 g}\right) \in \mathbb{R}^{2 g}$, (identifying $S U(2)$ with the unit quaternions) the assignment $x_{n} \mapsto e^{2 \pi i \phi_{n}}, \tau \mapsto j$ defines a special representation of $\pi_{1} M$ if and only if $\phi(B+I) \in \mathbb{Z}^{2 g}$ (Proposition 5.5 of [10]). Furthermore, every special representation is conjugate to one of this form. We will denote this representation by $\rho_{\phi}$. (If $F$ is a torus, then every nonabelian representation is conjugate to a special representation.)

Notice that replacing $\phi$ by $\pm \phi+v$ for $v \in \mathbb{Z}^{2 g}$ gives a conjugate representation.

If $h: F \rightarrow F$ is a homeomorphism, and $\left\{y_{k}\right\}$ is the new basis of $\pi_{1} F$ defined by $h\left(x_{k}\right)=y_{k}$, then $\rho_{\phi}\left(y_{k}\right)=e^{2 \pi i \phi_{k}^{\prime}}$ where $\phi^{\prime}=\phi H$. Here $H$ is the matrix induced by $h$ on homology (with respect to the basis $\left.\left\{x_{k}\right\}_{k=1}^{2 g}\right)$. (Invariantly, $\phi$ is an element of $H^{1}(F ; \mathbb{R})$ which satisfies $m^{*}(\phi)-\phi \in H^{1}(F ; \mathbb{Z})$.)

Let $\gamma$ be a simple closed curve in $F$. Identify $F$ with $F \times\{0\}$, and view $\gamma$ as a knot in $M$. Then $M-\gamma$ is obtained by gluing $(F-\gamma) \times 0$ to $F \times 1$ using $m$. Therefore,

$$
\left.\pi_{1}(M-\gamma)=\left\langle\pi_{1} F, \tau\right| \tau z \tau^{-1}=m(z) \text { for each } z \in \pi_{1}(F-\gamma)\right\rangle .
$$


Lemma 7.1. Let $\phi$ and $\theta$ be two vectors in $\mathbb{R}^{2 g}$ which defines special representations $\rho_{\theta}$ and $\rho_{\phi}$ of $\pi_{1} M$. (So $\phi(B+I) \in \mathbb{Z}^{2 g}$ and $\theta(B+I) \in$ $\mathbb{Z}^{2 g}$.) Then there exists a simple closed curve $\gamma$ on $F$ so that for each $t \in[0,1]$, the assignment $x_{n} \mapsto e^{2 \pi i\left((1-t) \phi_{n}+t \theta_{n}\right)}, \tau \mapsto j$ defines a path of representations of $M-\gamma$ from $\rho_{\phi}$ to $\rho_{\theta}$.

Proof. If $(\theta-\phi)(B+I)=0$, then $(t(\theta-\phi)+\phi)(B+I) \in \mathbb{Z}^{2 g}$ for all $t$, and so $(1-t) \phi+t \theta$ defines a path of representations of $\pi_{1} M$ from $\rho_{\phi}$ to $\rho_{\theta}$. Thus assume $(\theta-\phi)(B+I) \neq 0$.

Endow $\mathbb{Z}^{2 g}$ with the standard symplectic pairing. There exists a symplectic matrix $H$ such that $(\theta-\phi)(B+I) H=(n, 0, \cdots, 0)$ for some integer $n$ since any primitive vector in $\mathbb{Z}^{2 g}$ is the first vector of a symplectic basis. Choose a homeomorphism $h: F \rightarrow F$ so that $h_{*}=H$ in the basis $\left\{x_{k}\right\}$. The representations $\rho_{\phi}$ and $\rho_{\theta}$ are expressed in the basis $y_{k}=H x_{k}$ by $\phi^{\prime}=\phi H$ and $\theta^{\prime}=\theta H$. Moreover $B$ is changed to $B^{\prime}=H^{-1} B H$. Thus

$$
\left(\theta^{\prime}-\phi^{\prime}\right)\left(B^{\prime}+I\right)=(\theta-\phi)(B+I) H=(n, 0, \cdots, 0) .
$$

Let $\gamma=y_{2}$. Then $H_{1}(F-\gamma)$ is generated by $y_{2}, y_{3}, \cdots, y_{2 g}$. Using the remark preceding the lemma it is therefore easy to check that $y_{k} \mapsto$ $e^{2 \pi i\left((1-t) \phi_{k}+t \theta_{k}\right)}, \tau \mapsto j$ defines a path of representations of $\pi_{1}(M-\gamma)$ from $\rho_{\phi}$ to $\rho_{\theta}$. q.e.d.

The proof shows how to find the desired curve: First, choose a symplectic matrix $H$ so that $(\theta-\phi)(B+I) H=(n, 0, \cdots, 0)$. Then choose a homeomorphism inducing $H$ on homology, and let $\gamma \subset F$ be a simple closed curve representing $\mathrm{Hx}_{2}$.

In particular, if $M$ is a torus bundle, we have

Corollary 7.2. Let $M$ be a torus bundle with monodromy matrix $B$, and let $\rho_{\phi}, \rho_{\theta}: \pi_{1} M \rightarrow S U(2)$ be special representations corresponding to $\phi=\left(\phi_{1}, \phi_{2}\right)$ and $\theta=\left(\theta_{1}, \theta_{2}\right)$. Then $(1-t) \phi+t \theta$ defines a path of representations of $\pi_{1}(M-\gamma)$ where $\gamma=p x_{1}+q x_{2},(p, q)$ is a relatively prime pair satisfying

$$
(\phi-\theta)(B+I)\left(\begin{array}{l}
p \\
q
\end{array}\right)=0 .
$$

Moreover, (1) uniquely determines $\gamma$ (as an unoriented curve) up to isotopy in terms of $\theta$ and $\phi$ if $\operatorname{det}(B+I) \neq 0$.

Proof. All but the last statement follow from the previous lemma. The reason why (1) uniquely determines $\gamma$ as an unoriented curve is that given any vector $v=\left(v_{1}, v_{2}\right) \in \mathbb{Z}^{2}$, there is a unique (up to sign) primi- 
tive vector $w \in \mathbb{Z}^{2}$ orthogonal to $v$, namely $\left(-v_{2}, v_{1}\right) / \operatorname{gcd}\left(v_{1}, v_{2}\right)$. So $(\phi-\theta)(B+I)=n(-q, p)$ with $\operatorname{gcd}(p, q)=1$. q.e.d.

We set up some notation which will be used for the rest of this section. We use $B \in S L_{2}(\mathbb{Z})$ to denote the monodromy and take:

$$
B=\left(\begin{array}{ll}
a & b \\
c & d
\end{array}\right)
$$

Given $\theta, \phi \in \mathbb{R}^{2}$ determining special representations $\rho_{\theta}$ and $\rho_{\phi}$, let $\gamma$ be the corresponding knot in $M$. Let $X_{\gamma}$ be the complement of a tubular neighborhood of $\gamma$. Let

$$
\theta(t)=\left(\theta_{1}(t), \theta_{2}(t)\right)=(1-t) \theta+t \phi,
$$

and let $\rho_{t}: \pi_{1} X_{\gamma} \rightarrow S U(2)$ be the path of representations given by

$$
\tau \mapsto j, \quad x \mapsto e^{2 \pi i \theta_{1}(t)}, \quad y \mapsto e^{2 \pi i \theta_{2}(t)} .
$$

The fundamental group of $X_{\gamma}$ is

$$
\pi_{1} X_{\gamma}=\left\langle x, y, \tau \mid[x, y]=1, \tau x^{p} y^{q} \tau^{-1}=x^{p a+q b} y^{p c+q d}\right\rangle .
$$

The meridian of $X_{\gamma}$ (i.e., the boundary of a disk fiber in the tubular neighborhood of $\gamma$ in $M$ ) is represented by the element

$$
\mu=x^{r a+s b} y^{r c+s d} \tau x^{-r} y^{-s} \tau^{-1},
$$

where $r$ and $s$ are any integers satisfying $r q-p s=1$. We can take a longitude to be any complementary curve to $\mu$; a convenient choice is $\lambda=x^{p} y^{q}$. Figure 3 explains these choices.

Finally let $\Lambda \subset \mathbb{R}^{2}$ denote the half-integer lattice $\Lambda=\left(\frac{1}{2} \mathbb{Z}\right)^{2}$.

Given a conjugacy class $\left[\rho_{0}\right]$ in $R(M)$ of nonabelian representations, there is a $\theta \in \mathbb{R}^{2}$ so that $\rho_{0}$ is conjugate to $\rho_{\theta}$. Of course $\theta$ is not unique, it may be replaced by $\pm \theta+v$ for any $v \in \mathbb{Z}^{2}$. Given two conjugacy classes $\left[\rho_{0}\right]$ and $\left[\rho_{1}\right]$, a choice of $\theta$ and $\phi$ in $\mathbb{R}^{2}$ so that $\rho_{0} \sim \rho_{\theta}$ and $\rho_{1} \sim \rho_{\phi}$ determines the curve $\gamma \subset T^{2} \subset M$ via (1). In general, different choices of $\theta$ and $\phi$ will give a different curve $\gamma$, and the resulting paths $\rho_{t}$ given by (2) may have different properties.

Lemma 7.3. In the notation of the preceding paragraphs:

1. The representation $\rho_{t}$ is abelian if and only if $\theta(t) \in \Lambda$.

2. The restriction of $\rho_{t}$ to $\partial X_{\gamma}$ is given by

$$
\begin{aligned}
& \rho_{t}(\mu)=\exp \left(2 \pi i\left((r(a+1)+s b) \theta_{1}(t)+\left(r c+s(d+1) \theta_{2}(t)\right)\right)\right. \\
& \text { and } \\
& \qquad \rho_{t}(\lambda)=\exp \left(2 \pi i\left(p \theta_{1}(t)+q \theta_{2}(t)\right)\right) .
\end{aligned}
$$




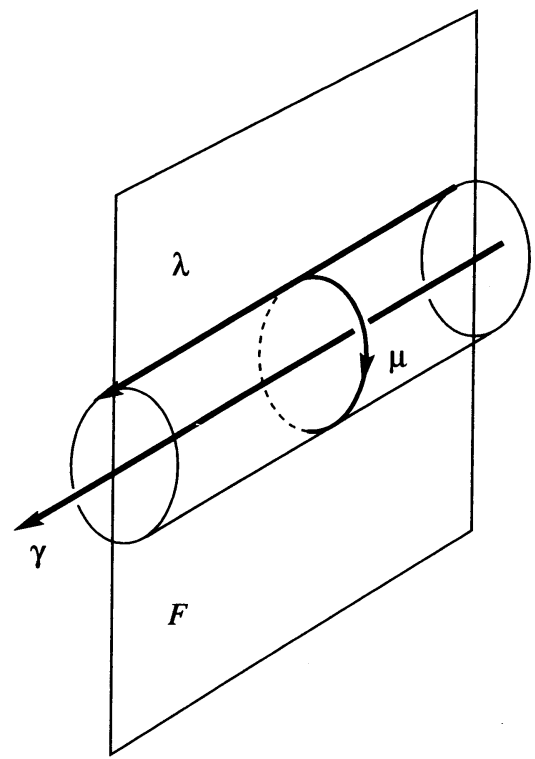

FIGURE 3

In particular, the restriction of $\rho_{t}$ to the boundary $\partial X_{\gamma}$ is central if and only if

$$
\theta(t)\left((B+I)\left(\begin{array}{l}
r \\
s
\end{array}\right),\left(\begin{array}{l}
p \\
q
\end{array}\right)\right) \in \Lambda .
$$

Moreover, if $\rho_{t}$ is abelian, then the restriction of $\rho_{t}$ to the boundary is central.

Proof. 1. Since $\rho_{t}(\tau)=j, \rho_{t}$ is abelian if and only if $\rho_{t}(x)$ and $\rho_{t}(y)$ are both central. Since $\rho_{x}(x)=e^{2 \pi i \theta_{1}(t)}$ and $\rho_{t}(y)=e^{2 \pi i \theta_{y}(t)}$, this can happen only if $\theta(t) \in \Lambda$.

2. $\rho_{t}(\mu)=\rho_{t}\left(x^{r a+s b} h^{r c+s d} \tau x^{-r} y^{-s} \tau^{-1}\right)=\rho_{t}\left(x^{r a+s b} y^{r c+s d} x^{r} y^{s}\right)$. Since $x$ and $y$ commute, (1) clearly holds, and (2) is obvious. q.e.d.

In order to apply the theorems of the previous sections, one needs paths of representations of $X_{\gamma}$ so that the restriction to the boundary is not central along the path. This is because when the path passes through a representation whose restriction to the boundary is central, then the symplectic space $\mathscr{H}$ jumps up in dimension from 4 to 12 .

Definition 7.4. Given conjugacy classes $\left[\rho_{0}\right],\left[\rho_{1}\right]$ of representations of $\pi_{1} M$, we will say that $\theta, \phi \in \mathbb{R}^{2}$ form a good pair for $\left[\rho_{0}\right],\left[\rho_{1}\right]$ if $\rho_{0}$ is conjugate to $\rho_{\theta}, \rho_{1}$ is conjugate to $\rho_{\phi}$, and the path $\rho_{t}$ defined by (2) restricts to a path of noncentral representations of $\pi_{1} X_{\gamma}$. 
In every example which the authors tried, it was always possible to find a good pair given any two conjugacy classes of representations of $\pi_{1} M$. We do not know a proof that such a path can always be found, but we conjecture that one always exists. Finding a good pair is a technical problem of linear algebra over $\mathbb{Z}$. Proving that $\theta$ and $\phi$ can be chosen so that $\rho_{t}$ is nonabelian for all $t$ is easier. For example, a fundamental domain for the $(\mathbb{Z} \oplus \mathbb{Z}) \rtimes(\mathbb{Z} / 2)$ action on $\mathbb{R}^{2}$ given by $\left(m_{1}, m_{2}, \pm 1\right)\left(\theta_{1} \theta_{2}\right)= \pm\left(\theta_{1}+m_{1}, \theta_{2}+m_{2}\right)$ is the set

$$
D=\left([0,1) \times\left(0, \frac{1}{2}\right)\right) \cup\left(\left[0, \frac{1}{2}\right] \times\{0\}\right) \cup\left(\left[0, \frac{1}{2}\right] \times\left\{\frac{1}{2}\right\}\right) .
$$

If we choose $\theta$ and $\phi$ in $D$, they lie in $D-\Lambda$ since $\rho_{\theta}$ and $\rho_{\phi}$ are nonabelian. But then the entire path $(1-t) \theta+t \phi$ lies in $D-\Lambda$, and so by the previous lemma this determines a path of nonabelian representations.

We can now state the main result of this section. This is a computation of the spectral flow mod 4 between two nonabelian representations of a torus bundle over $S^{1}$. This theorem verifies Conjecture 5.8 of [8] for the case $G=S U(2)$ at least when a good pair of representatives can be found for a pair of representations.

Theorem 7.5. Let $M$ be a mapping torus with fiber $T^{2}$ and monodromy matrix $B$. Assume that \pm 1 are not eigenvalues of $B$. Let $\left[\rho_{0}\right]$, $\left[\rho_{1}\right] \in R(M)$ be two conjugacy classes of nonabelian representations, and assume that a good pair $\theta, \phi \in \mathbb{R}^{2}$ for $\rho_{0}, \rho_{1}$ can be found. Then the spectral flow of the Hessian of the Chern-Simons function between $\rho_{0}$ and $\rho_{1}$ is congruent to 0 mod 4.

Before we embark on a proof of this theorem, we first do a few computations to show the reader how to find all nonabelian representations of a torus bundle, and how to find paths which satisfy the technical restrictions of this theorem.

We start with a simple example. Take the monodromy matrix

$$
B=\left(\begin{array}{cc}
3 & 1 \\
-1 & 0
\end{array}\right)
$$

The determinant of $B+I$ is 5 . Since every nonabelian representation is conjugate to a special representation $\rho_{0}$ with $\theta \in D-\Lambda$ ( $D$ is the fundamental domain described above), the possible values of $\theta$ lie in $\left(\frac{1}{5} \mathbb{Z}\right)^{2} \cap(D-\Lambda)$. This intersection contains 12 points. One easily checks that of these twelve points, only $\left(\frac{3}{5}, \frac{2}{5}\right)$ and $\left(\frac{4}{5}, \frac{1}{5}\right)$ satisfy $\theta(B+I) \in \mathbb{Z}^{2}$. Thus there are two conjugacy classes of nonabelian representations of $M$. To apply Theorem 7.5, we must check that $\theta$ and $\phi$ form a good pair. Let $\theta=\left(\frac{3}{5}, \frac{2}{5}\right)$ and $\phi=\left(\frac{4}{5}, \frac{1}{5}\right)$; then $(\theta-\phi)(B+I)=(1,0)$. Therefore, 
$\gamma=(p, q)=(0,1)$. Hence:

$$
\begin{aligned}
\theta(t)\left((B+I)\left(\begin{array}{l}
r \\
s
\end{array}\right),\left(\begin{array}{l}
p \\
q
\end{array}\right)\right) & =\left((1-t \theta+t \phi)\left((B+I)\left(\begin{array}{l}
1 \\
0
\end{array}\right),\left(\begin{array}{l}
0 \\
1
\end{array}\right)\right) .\right. \\
& =\left(2, \frac{2}{5}\right)+t\left(1,-\frac{1}{5}\right) .
\end{aligned}
$$

This does not pass through $\Lambda$ for $t \in[0,1]$. According to Lemma 7.3 this implies that $\rho_{t}$ restricts to a noncentral representation for each $t \in[0,1]$, i.e., $\theta$ and $\phi$ form a good pair. Theorem 7.5 now implies that the spectral flow from $\rho_{\theta}$ to $\rho_{\phi}$ is congruent to $0 \bmod 4$.

This example has $H_{1}(M ; \mathbb{Z})=\mathbb{Z}$, and the abelianization is generated by $\tau$. Therefore the space of conjugacy classes of abelian representations is an arc. A parameterization is given by the path of representations:

$$
\rho_{s}(\tau)=e^{i s}, \quad \rho_{s}(x)=\rho_{s}(y)=1, \quad s \in[0, \pi] .
$$

Moreover, this arc is a smooth component of $R(M)$ except at its endpoints (a calculation similar to those of Lemma 7.7 below shows that $H^{1}\left(M ; p \operatorname{ad} \rho_{s}\right)=\mathbb{R}$ for all $\left.s \in(0, \pi)\right)$. Therefore, the spectral flow between $\rho_{s}$ and $\rho_{s^{\prime}}$ is $-1 \bmod 8$ (using Floer's convention of counting the intersections of the graph of the spectrum with a line from $(0,-\varepsilon)$ to $(1, \varepsilon))$. Computing the spectral flow to the trivial representation requires computing the eigenvalues on the bilinear form at the trivial representation. This is done in general in Theorem 7.9 below.

As a second example we find a bad (i.e., not good) pair $\theta$ and $\phi$. Let $M$ be a mapping torus with monodromy:

$$
B=\left(\begin{array}{ll}
7 & 5 \\
4 & 3
\end{array}\right) \text {. }
$$

Then $\pi_{1} M$ has 5 nonabelian representations, which can be found in the same way as the first example. Among these are the representations $\rho_{\theta}$ and $\rho_{\phi}$ corresponding to $\theta=\left(0, \frac{1}{4}\right)$ and $\phi=\left(\frac{1}{3}, \frac{1}{12}\right)$.

With these choices of $\theta$ and $\phi$, we see that $(\theta-\phi)(B+I)=(-2,-1)$, and so the curve $\gamma$ to be removed is $\gamma=(-1,2)$. But with this choice of $\gamma, \rho_{\theta}(\mu)=1$ and $\rho_{\theta}(\lambda)=-1$, as is readily checked. Thus $\theta$ and $\phi$ do not form a good pair, and so Theorem 7.5 does not apply. However $\theta$ can be replaced by $\pm \theta+v$ for $v \in \mathbb{Z}^{2}$, and similarly for $\phi$ without changing their conjugacy class. We can try a different choice to see if we get a good pair.

Indeed, with the choices $\hat{\theta}=\left(1, \frac{1}{4}\right)=\left(0, \frac{1}{4}\right)+(1,0)$ and $\hat{\phi}=\phi=$ $\left(\frac{1}{3}, \frac{1}{12}\right)$,

$$
(\hat{\theta}-\hat{\phi})(B+I)=(6,4) \text {. }
$$


Thus the knot to be removed is $\gamma=(2,-3)$. Arithmetic gives:

$$
\rho_{t}(\mu)=e^{2 \pi i(3-2 t)}, \quad \rho_{t}(\lambda)=e^{2 \pi i(5 / 4-5 t / 6)},
$$

which does not pass through any central representation for $t \in[0,1]$. So $\hat{\theta}$ and $\hat{\phi}$ form a good pair. Hence Theorem 7.5 applies to this path, and the spectral flow from $\rho_{\theta}$ to $\rho_{\phi}$ (which of course is independent of the representatives $\theta$ and $\phi$ ) is congruent to $0 \bmod 4$ after all. In this example the spectral flow between any two nonabelian representations is congruent to $0 \bmod 4$.

We begin the proof of Theorem 7.5 with some cohomology calculations. We will use the usual model for group cohomology, so $C^{0}(\pi, s u(2))=$ $s u(2)$ and $C^{n}(\pi, s u(2))=\operatorname{Maps}\left(\pi^{n}, s u(2)\right)$. Moreover, $\delta^{0}(v)(x)=v-$ $x \cdot v$ for $v \in s u(2)$ and $\delta^{1}(f)(x, y)=f(x)+x \cdot f(y)-f(x y)$, where $x \cdot v$ means ad $\rho(x)(v)$. Identify $s u(2)$ with the imaginary quaternions, and write $s u(2)=\mathbb{R} i \oplus \mathbb{C} j$. The cup product is defined as follows: if $a, b$ are 1-cochains, and $x, y \in \pi$, then

$$
[a, b](x, y)=[a(x), x \cdot b(y)] .
$$

If $c$ is a 0 -cochain, then

$$
[a, c](x)=[a(x), x \cdot c] .
$$

We refer to [3] for details.

Lemma 7.6. Let $M$ be a torus bundle over $S^{1}$, with monodromy matrix $B$. Assume that -1 is not an eigenvalue of $B$. Let $\rho$ be a nonabelian representation. Then $H^{0}(M ;$ ad $\rho)$ and $H^{1}(M ;$ ad $\rho)$ are both zero.

Proof. We continue using the notation described before Lemma 7.3. Note that $H^{i}(M ;$ ad $\rho)=H^{i}\left(\pi_{1} M\right.$; ad $\left.\rho\right)$ for $i=0,1$, so we can compute the cohomology of $\pi_{1} M$ instead of $M$. We may assume, by conjugating, that $\rho=\rho_{\theta}$ for $\theta=\left(\theta_{1}, \theta_{2}\right) \in \mathbb{R}^{2}$.

For $t, u$ and $v$ real numbers, the coboundary $\delta^{0}(t i+(u+v i) j): \pi \rightarrow$ $\mathbb{R} i \oplus \mathbb{C} j$ sends $x$ to $\left(1-e^{4 \pi i \theta_{1}}\right)(u+v i) j, y$ to $\left(1-e^{4 \pi i \theta_{2}}\right)(u+v i) j$, and $\tau$ to $2 t i+2 v k$. Let $z$ be a 1-cocycle. Write $z(x)=X, z(y)=Y$, and $z(\tau)=T$. Applying the cocycle formula to the first relation we see that

$$
0=X+x \cdot Y-x y x^{-1} \cdot X-x y x^{-1} y^{-1} Y=(1-y) \cdot X+(x-1) \cdot Y \text {. }
$$

If $\theta_{1}$ is not a half-integer, then by subtracting a coboundary from $z$ we may assume that $X \in \mathbb{R} i$. But then the relation implies that $x-1$ acts trivially on $Y$, so that $Y$ is also in $\mathbb{R} i$. Similarly if $\theta_{2}$ is not a halfinteger, we may change $z$ by a coboundary so that $X$ and $Y$ are both 
in $\mathbb{R} i$. One of the $\theta_{k}$ is not a half-integer since we are assuming that $\rho_{\theta}$ is a nonabelian representation, and so it cannot restrict to a central representation of $\pi_{1} F$. We can further change $z$ by a coboundary so that $T$ is in $\mathbb{C} j$ by subtracting $\delta^{0}(a i)$; this does not affect $X$ or $Y$.

With these simplifications, the other two relations $x^{a} y^{c}=\tau x \tau^{-1}, x^{g} y^{d}$ $=\tau y \tau^{-1}$ imply that

$$
(B+I)\left(\begin{array}{l}
X \\
Y
\end{array}\right)=0 \text { and } T=0 .
$$

For example, if $a, c>0$, then the relation $x^{a} y^{c} \tau x^{-1} \tau=1$ implies that

$$
\begin{aligned}
0= & \left(1+x+\cdots x^{a-1}\right) \cdot X+x^{a}\left(1+y+\cdots+y^{c-1}\right) \cdot Y \\
& +x^{a} y^{c} T-x^{a} y^{c} \tau x^{-1} X-x^{a} y^{c} \tau x^{-1} \tau^{-1} T \\
= & (a+1) X+c Y+\left(\tau x \tau^{-1}-1\right) \cdot T .
\end{aligned}
$$

Comparing $\mathbb{R} i$ coefficients gives $(a+1) X+c Y=0$. Comparing the $\mathbb{C} j$ coefficients gives $0=\left(\tau x \tau^{-1}-1\right) \cdot T=\left(x^{-1}-1\right) \cdot T=\left(e^{-4 \pi i \theta_{1}}-1\right) T$. If $\theta_{1}$ is not a half-integer, this implies $T=0$. A similar calculation works when $a$ and $c$ are not both positive and for the other relation $x^{b} y^{d} \tau y^{-1} \tau=1$.

Since $\operatorname{det}(B+I) \neq 0$ by hypothesis, $X$ and $Y$ are both zero. So every 1-cocycle is cohomologous to 0 and $H^{1}(M ; \operatorname{ad} \rho)=0$. q.e.d.

We next turn to the computation of the cohomology of $M-\gamma$. As a preliminary remark we observe that a choice of generators $\mu, \lambda$ of $\pi_{1} T^{2}$ determines a canonical isomorphism $\mathbb{R}^{2} \cong H^{1}\left(\pi_{1} T\right.$; ad $\left.\rho\right)$ for any noncentral representation $\rho$. Indeed, the isomorphism takes the pair $(u, v)$ to the 1-cocycle $z$ whose value on $\mu$ is $i u$ and whose value on $\lambda$ is $i v$. In particular, this identification is independent of the choice of representative of a conjugacy class. Given any 3-manifold $X$ with boundary $T^{2}$ and any representation $\rho$ whose restriction to the boundary is noncentral, the image $H^{1}(X ; \operatorname{ad} \rho) \rightarrow H^{1}(\partial X ; \operatorname{ad} \rho)$ is a 1-dimensional subspace (a Lagrangian with respect to the cup product), and so it makes sense to define the slope of this image, and moreover to compare the slopes at different representations.

In the next lemma, we continue with the usual hypotheses: $M$ is a torus bundle with monodromy matrix $B,-1$ is not an eigenvalue of $B$, $\theta, \phi \in \mathbb{R}^{2}$ are a good pair corresponding to two nonabelian special representations, $\gamma$ is a $(p, q)$ curve in the fiber so that $(\theta-\phi)(B+I)(p, q)^{t}=0$ and $\rho_{t}: \pi_{1} X_{\gamma} \rightarrow S U(2)$ is the corresponding path of representations.

Finally $\mu$ and $\lambda$ are the generators for $\pi_{1} \partial X_{\gamma}$ described before Lemma 7.3. We refer to the slope with respect to these generators. With this 
notation fixed, we have:

\section{Lemma 7.7.}

1. $H^{0}\left(X_{\gamma} ; \operatorname{ad} \rho_{t}\right)=0$.

2. $H^{1}\left(X_{\gamma} ;\right.$ ad $\left.\rho_{t}\right) \cong \mathbb{R}$ if $\rho_{t}(\lambda) \neq \pm 1$ and $H^{1}\left(X_{\gamma} ; \operatorname{ad} \rho_{t}\right) \cong \mathbb{R}^{3}$ if $\rho_{t}(\lambda)= \pm 1$.

3. The slope of the image $H^{1}\left(X_{\gamma} ;\right.$ ad $\left.\rho_{t}\right) \rightarrow H^{1}\left(\partial X_{\gamma} ; \operatorname{ad} \rho_{t}\right)$ is independent of $t \in[0,1]$. (Notice that this image is the differential of the restriction map $R\left(X_{\gamma}\right) \rightarrow R\left(\partial X_{\gamma}\right)$.) Using $\mu, \lambda$ to identify $H^{1}\left(\partial X_{\gamma}\right.$; ad $\left.\rho_{t}\right)$ with $\mathbb{R}^{2}$, this image is

$$
\left\{(b u+(d+1) v, u) \in \mathbb{R}^{2} \mid(a+1) u+c v=0\right\} .
$$

\section{Remarks.}

1. The image

$$
\begin{aligned}
H^{0}\left(X_{\gamma}, \partial X_{\gamma} ; \operatorname{ad} \rho_{t}\right) & \oplus H^{1}\left(X_{\gamma}, \partial X_{\gamma} ; \operatorname{ad} \rho_{t}\right) \\
& \rightarrow H^{0}\left(X_{\gamma} ; \operatorname{ad} \rho_{t}\right) \oplus H^{1}\left(X_{\gamma} ; \operatorname{ad} \rho_{t}\right)
\end{aligned}
$$

is zero if $\rho_{t}(\lambda) \neq \pm 1$, and is 2-dimensional if $\rho_{t}(\lambda)= \pm 1$. This means that if $A_{t}$ is a path of flat connections on $X_{\gamma}$ with holonomy $\rho_{t}$, then the corresponding path of operators $\mathscr{D}_{A_{t}}$ constructed in $\S 4$ has kernel only at those isolated $t$ when $\rho_{t}(\lambda)= \pm 1$, and that the kernel is 2-dimensional at these points. We will use Theorem 4.8 to compute the spectra flow of $\mathscr{D}_{t}$ as $t$ passes through such a point $t_{0}$ in terms of the eigenvalues of a bilinear form on $H^{1}\left(X_{\gamma} ;\right.$ ad $\left.\rho_{t_{0}}\right)$. Notice that $\rho_{t}(\lambda) \neq \pm 1$ for $t=0$ or 1 .

2. The restriction of the path of representations of $X_{\gamma}$ to the boundary is linear (as a path in $\mathbb{R}^{2}$ ). In fact this path is given by

$$
f(t)=\theta(t)\left((B+I)\left(\begin{array}{l}
r \\
s
\end{array}\right),\left(\begin{array}{l}
p \\
q
\end{array}\right)\right) .
$$

In particular $f$ is an immersion, as required by Theorem 6.3. We pick the Lagrangians for Theorem 2.1 to be

$$
\begin{aligned}
\mathscr{L}_{1} & =\mathbb{R} i \oplus * \operatorname{span} \frac{d f(t)}{d t} \oplus 0 \\
& =\sigma\left(0 \oplus \operatorname{im}\left(H^{1}\left(X_{\gamma} ; \operatorname{ad} \rho_{t}\right) \rightarrow H^{1}\left(\partial X_{\gamma} ; \operatorname{ad} \rho_{t}\right)\right) \oplus \mathbb{R} i d x d y\right)
\end{aligned}
$$

for $M_{1}=X_{\gamma}$, and $\mathscr{L}_{2}=0 \oplus\{(v, 0)\} \mathbb{R} i$ as in $\S 6$.

Thus all the hypotheses of Theorems 4.8 and 6.4 are satisfied. 
Proof. Notice that $\rho_{t}$ is a path of nonabelian representations, since if $\rho_{t_{0}}$ is abelian for some $t_{0}$, then by Lemma 7.3 the restriction of $\rho_{t_{0}}$ to the boundary is central, contradicting the hypothesis. Therefore $H^{0}\left(X_{\gamma} ; \operatorname{ad} \rho_{t}\right)$ $=0$.

By changing coordinates on the torus we may assume that $\gamma$ is the $(1,0)$ curve in the fiber. Then $\pi_{1}\left(X_{\gamma}\right)=\left\langle x, y, \tau \mid \tau x \tau^{-1}=x^{a} y^{c}\right\rangle$ and hence $\mu=x^{b} y^{d} \tau y^{-1} \tau^{-1}$ and $\lambda=x$.

Let $z$ be a 1-cocycle taking $x$ to $X, y$ to $Y$ and $\tau$ to $T$. As in the proof of Lemma 7.6, we may change $z$ by a coboundary so that $X$ and $Y$ are in $\mathbb{R} i$ and $T$ is in $\mathbb{C} j$. This normalization specifies $z$ uniquely in its cohomology class.

The cocycle formula applied to $z\left(x^{a} y^{c} \tau x^{-1} \tau^{-1}\right)=0$ implies

$$
(a+1) X+c Y+\left(x^{-1}-1\right) \cdot T=0 .
$$

Thus $(a+1) X+c Y=0$ and $\left(e^{-4 \pi i \theta_{1}(t)}-1\right) T=0$. This first equation defines a 1-dimensional subspace of $(i \mathbb{R})^{2}$ since $\operatorname{det}(B+I) \neq 0$. The second equation implies that $T=0$ if $\theta_{1}(t) \notin \frac{1}{2} \mathbb{Z}$, i.e., if $\rho_{t}(\lambda) \neq \pm 1$. On the other hand, if $\rho_{t}(\lambda)= \pm 1$, then this equation puts no restriction on $T$, and so $T$ can be arbitrary. This proves the second assertion.

For the last assertion, let $z$ be a cocycle satisfying $z(x)=X \in i \mathbb{R}$, $z(y)=Y \in i \mathbb{R}$, and $z(\tau)=0$. So $(a+1) X+c Y=0$. Then one checks that $z(\mu)=z\left(x^{b} y^{d} \tau y^{-1} \tau^{-1}\right)=b X+(d+1) Y$ and $z(\lambda)=z(x)=X$. Use $\mu$ and $\lambda$ to identify $H^{1}\left(\partial X_{\gamma} ;\right.$ ad $\left.\rho_{t}\right)=\mathbb{R}^{2}$ (see the paragraph preceding this lemma). Then the image $\operatorname{im}\left(H^{1}\left(X_{\gamma} ; \operatorname{ad} \rho_{t}\right) \rightarrow H^{1}\left(\partial X_{\gamma} ; \operatorname{ad} \rho_{t}\right)=\mathbb{R}^{2}\right)$ contains the set $\left\{(b u+(d+1) v, u) \in \mathbb{R}^{2} \mid(a+1) u+c v=0\right\}$. This set is a 1-dimensional subspace since $\operatorname{det}(B+I) \neq 0$, and so must be the entire image. Furthermore, it is independent of $t$. q.e.d.

If $\rho_{t}(\lambda)= \pm 1$, then the 1-cocycle $z$ defined by $z(x)=0=z(y)$ and $z(\tau)=T$ restricts to a coboundary on $\partial X_{\gamma}$. Thus these " $\mathbb{C} j$ " cocycles represent the image of $H^{1}\left(X_{\gamma}, \partial X_{\gamma} ; \operatorname{ad} \rho_{t}\right)$ in $H^{1}\left(X_{\gamma} ; \operatorname{ad} \rho_{t}\right)$, which is isomorphic to the space of $L^{2}$ solutions to $D_{A} \phi=0$.

We can now prove Theorem 7.5. The bulk of the proof is computing the bilinear form of Theorem 4.8, using group cohomology.

Proof of Theorem 7.5. Let $Y$ be a tubular neighborhood of $\gamma$, so that $M=X_{\gamma} \cup_{T} Y$. The path $\rho_{t}$ described by equation (2) defines an analytic path of flat connections $A_{t}$ on $X_{\gamma}$; in fact this path is of the form $d_{0}+t A$, where $d_{0}$ is the flat connection with holonomy $\rho_{0}$, and $A$ is a $d_{0}$-harmonic 1 -form representing the generator of $H^{1}\left(X_{\gamma} ; \operatorname{ad} \rho_{0}\right)$. 
Moreover, $A_{t}$ extends to a path of connections on $M$ which are flat when $t=0$ or $t=1$.

Let $f: I \rightarrow \mathbb{R}^{2}$ be the path

$$
f(t)=\theta(t)\left((B+I)\left(\begin{array}{l}
r \\
s
\end{array}\right),\left(\begin{array}{l}
p \\
q
\end{array}\right)\right) .
$$

So $\rho_{t}(\mu)=e^{2 \pi i f_{1}(t)}$ and $\rho_{t}(\lambda)=e^{2 \pi i f_{2}(t)}$.

By hypothesis $f(t)$ does not pass through any points of $\left(\frac{1}{2} \mathbb{Z}^{2}\right)$.

According to Theorem 2.1, the spectral flow is the sum of three terms, $S F\left(\rho_{0}, \rho_{1} ; M\right)=S F\left(\mathscr{D}_{t} ; X_{\gamma}\right)+S F\left(\mathscr{D}_{t} ; Y\right)+\gamma\left(\mathscr{L}_{t}, \mathscr{K}_{t}\right)$. Theorem 6.4 implies that the sum of the second and third terms is computed as follows: each intersection of $f(t)$ with a vertical line of the form $x=(2 k+$ 1) $/ 2, \quad i \in \mathbb{Z}$, contributes 4 and each intersection with a horizontal line $y=k / 2, k \in \mathbb{Z}$ contributes $\pm 2(\bmod 8)$. There is no "writhe" term since $f(t)$ is a straight line, and so the sum of the second and third term in the formula equals the sum of these contributions.

To complete the proof, then, we will show that the first term in the formula of Theorem 2.1 is the sum of \pm 2 , where the sum is taken over each intersection of $f(t)$ with one of the horizontal lines $y=k / 2$. The first remark after Lemma 7.7 implies that $\mathscr{D}_{t}\left(X_{\gamma}\right)$ has kernel only when $\rho_{t}(\lambda)= \pm 1$, that is, only when $f(t)$ intersects one of these horizontal lines, and that this kernel is 2-dimensional.

We will use Theorem 4.8 to compute the spectral flow on the manifold $X_{\gamma}$ near each value of $t$ where one of these intersections occurs. To see that Theorem 4.8 applies, it is important to note that because the arc $\rho_{t}$ of representations is analytic in $t$, we may choose the corresponding arc of connections to be analytic in $t$. Also, because the representations restrict to abelian representations on $\partial X_{\gamma}$, these connections may be chosen to be in "standard form" (in the sense of Appendix B) near the boundary. Because of this the path of connections on the boundary may be assumed to be fine and hence we can apply Theorem 4.8 .

Theorem 4.8 states that the spectral flow near any value of $t$ is computed in terms of a certain bilinear form. Precisely, if $T_{0} \in[0,1]$ is a parameter value when $\rho_{t_{0}}(\lambda)= \pm 1$, let $U$ be the image of $H^{1}\left(X_{\gamma}, \partial X_{\gamma}\right.$; ad $\left.\rho_{t_{0}}\right)$ in $H^{1}\left(X_{\gamma} ; \operatorname{ad} \rho_{t_{0}}\right)$. Then $U$ is 2-dimensional and has a bilinear form $\left(u_{1}, u_{2}\right) \mapsto\left[u_{1}, u_{2}\right] \cdot a$, where $a \in H^{1}\left(X_{\gamma} ; \operatorname{ad} \rho_{t_{0}}\right)$ is the derivative of the path of connections. The spectral flow of $\mathscr{D}_{t}$ for $t \in\left(t_{0}-\varepsilon, t_{0}+\varepsilon\right)$ is the signature of this bilinear form. Thus we need to show that this form is definite so that the spectral flow is \pm 2 . Rather than compute this in the 
DeRham complex we can take the cellular complex, or, better, the group cohomology complex (since $X_{\gamma}$ and $\partial X_{\gamma}$ are $K(\pi, 1)$ 's).

In this context we have seen (Lemma 7.7) that $U$ is just the " $\mathbb{C} j$ " part of $H^{1}\left(X_{\gamma} ;\right.$ ad $\left.\rho_{t_{0}}\right)$. What this means is that the coefficients $s u(2)=\mathbb{R} i \oplus \mathbb{C} j$ split as a $\pi_{1} X_{\gamma}$-module (via the ad $\rho_{t_{0}}$ action), since if $\alpha \in \mathbb{R} i$,

$$
x \cdot \alpha=\alpha, \quad y \cdot \alpha=\alpha, \quad \tau \cdot \alpha=-\alpha
$$

and if $\alpha \in \mathbb{C} j$, then

$$
x \cdot \alpha=e^{2 \pi i \theta_{1}\left(t_{0}\right)} \alpha, \quad y \cdot \alpha=e^{2 \pi i \theta_{2}\left(t_{0}\right)} \alpha, \quad \tau \cdot \alpha=\bar{\alpha},
$$

where $\bar{w} j=\bar{w} j$ for $w \in \mathbb{C}$. Furthermore, the differentials preserve this splitting, since the values of $\delta z$ for a cochain $z$ are the same as the values of $z$. From (the proof of) Lemma 7.6 we see that a pair of generators of $U$ are the 1-cocycles $z_{1}$ and $z_{2}$ defined by

$$
z_{i}(x)=0=z_{i}(y), \quad z_{1}(\tau)=j, \quad z_{2}(\tau)=k .
$$

We will show that the bilinear form is $B\left(z_{i}, z_{j}\right)=R \delta_{i j}$ with respect to this basis, for some nonzero constant $R$.

In the expression $\left[u_{1}, u_{2}\right] \cdot a$, the element $a$ is the derivative of the path of flat connections. Translated to group cohomology, $a$ is the derivative of the path of representations. Since $\rho_{t}(x)=e^{2 \pi i \theta_{1}(t)}, \rho_{t}(y)=e^{2 \pi i \theta_{2}(t)}$, and $\rho_{t}(\tau)=j$, it follows that $a$ is the 1-cocycle defined by

$$
a(x)=4 \pi i \theta_{1}\left(t_{0}\right), \quad a(y)=4 \pi i \theta_{2}\left(t_{0}\right), \quad a(\tau)=0 .
$$

From (the proof of) Lemma 7.7 we see that $a$ is a generator of $H^{1}\left(X_{\gamma} ; \mathbb{R} i\right)$. (We hope the unfortunate choice of the letter $a$ to denote both this cohomology class and an entry in the matrix $B$ will not cause confusion.)

For notational convenience write $\theta_{i}\left(t_{0}\right)=\theta_{i}$, and change coordinates so that $\lambda=x$, hence $\theta_{1} \in \frac{1}{2} \mathbb{Z}$.

The cocycles $z_{i}$ are not relative cocycles; their restriction to the boundary does not vanish, but is merely a coboundary. Much of the technical work we do below is to work with explicit representatives which are relative cocycles. For the moment, choose $\tilde{z}_{i}$ to be 1-cocycles which vanish on the boundary; then $\left[\tilde{z}_{i}, \tilde{z}_{j}\right] \in H^{2}\left(X_{\gamma}, \partial X_{\gamma} ; \mathbb{R} i\right)$. The Poincaré duality, together with the fact that the product $\mathbb{R} i \otimes \mathbb{R} i \rightarrow \mathbb{R}$ given by $r \otimes s \mapsto r s$ is ad $\rho$-invariant and nondegenerate, implies that $H^{2}\left(X_{\gamma}, \partial X_{\gamma} ; \mathbb{R} i\right)=$ $H^{1}\left(X_{\gamma} ; \mathbb{R} i\right) \cong \mathbb{R}$. In particular,

$$
\cdot a: H^{2}\left(X_{\gamma}, \partial X_{\gamma} ; \mathbb{R} i\right) \rightarrow H^{3}\left(X_{\gamma}, \partial X_{\gamma} ; \mathbb{R}\right) \cong \mathbb{R}
$$


is an isomorphism. Therefore, to show that the bilinear form is definite we can choose any isomorphism $\alpha: H^{2}\left(X_{\gamma}, \partial X_{\gamma} ; \mathbb{R} i\right) \rightarrow \mathbb{R}$ and check that the bilinear form $\left(w_{1}, w_{2}\right) \mapsto \alpha\left(\left[w_{1}, w_{2}\right]\right)$ is definite.

The isomorphism we choose is the following: Given $u \in H^{2}\left(X_{\gamma}, \partial X_{\gamma}\right.$; $\mathbb{R} i)$, let $v_{u} \in H^{1}\left(\partial X_{\gamma} ; \mathbb{R} i\right)$ be a cocycle mapping to $u$ in the long exact sequence of the pair $\left(X_{\gamma}, \partial X_{\gamma}\right)$ (notice that $H^{2}\left(X_{\gamma} ; \mathbb{R} i\right)=0$ ). Then project $v_{u}$ to $H^{1}\left(\partial X_{\gamma} ; \mathbb{R} i\right) / H^{1}\left(X_{\gamma} ; \mathbb{R} i\right) \cong \mathbb{R}$. This approach has the advantage that we will only need to work with 1- and 2-cochains as well as the fact that we understand all the 1-dimensional cohomology groups.

The proof is computationally intensive, and so we will omit some straightforward but lengthy algebraic calculations.

First of all, our normalizations state that $\mu=x^{b} y^{d} \tau y^{-1} \tau^{-1}$ and $\lambda=x$. Using the 1-cocycle relation $z(r s)=z(r)+r \cdot z(s)$, one computes

$$
z_{1}(\mu)=y^{d}(1-y) j, \quad z_{1}(\lambda)=0, \quad z_{2}(\mu)=y^{d}(1-y) k, \quad z_{2}(\lambda)=0 .
$$

In these formulas, the symbol " $y$ " is used interchangeably for the element of $\pi_{1} X_{\gamma}$ and the complex number $e^{4 \pi i \theta_{2}}$. This is because the action of $y$ on $\mathbb{C} j$ is (left) multiplication by $e^{4 \pi i \theta_{2}}$.

We wish to subtract coboundaries $\delta v_{i}$ from $z_{i}$ to obtain $\tilde{z}_{i}=z_{i}-\delta v_{i}$ which vanish on the boundary. The correct choice is $v_{1}=\alpha j$ and $v_{2}=$ $\alpha k$, where $\alpha \in \mathbb{C}$ is defined as:

$$
\alpha=y^{d}(1-y) /\left(1-y^{d+1}\right) .
$$

Notice that this is well defined since if $e^{4(d+1) \pi i \theta_{2}}=1$, then since $\rho_{t_{0}}(\lambda)=$ \pm 1 , it follows that $\rho_{t_{0}}(\mu)= \pm 1$, contradicting the fact that the path is always noncentral on the boundary. A short computation shows that this is the correct choice.

Now the cup product is bilinear, so

$$
\left[\tilde{z}_{i}, \tilde{z}_{j}\right]=\left[z_{i}, z_{j}\right]-\left[z_{i}, \delta v_{j}\right]-\left[\delta v_{i}, z_{j}\right]+\left[\delta v_{i}, \delta v_{j}\right] .
$$

Suppose $s_{i j}$ and $r_{i j}$ are 1-cochains with values in $\mathbb{R} i$ so that $\delta s_{i j}=$ $\left[z_{i}, z_{j}\right]$ and $\delta r_{i j}=\left[\tilde{z}_{i}, \tilde{z}_{j}\right]$. Then

$$
\delta r_{i j}=\delta s_{i j}+\delta\left(\left[z_{i}, v_{j}\right]-\left[v_{i}, z_{j}\right]+\left[v_{i}, \delta v_{j}\right]\right)
$$

by the Leibnitz formula. Thus we can define

$$
r_{i j}=s_{i j}+\left[z_{i}, v_{j}\right]-\left[v_{i}, z_{j}\right]+\left[v_{i}, \delta v_{j}\right] .
$$

Notice that with this choice,

$$
r_{i j \mid \partial X_{y}}=s_{i j \mid \partial X_{y}}+\left[z_{i}, v_{j}\right]_{\mid \partial X_{y}}
$$


since the restriction of $\left[v_{i}, z_{j}-\delta v_{j}\right]$ to the boundary is zero. We will make a choice of $s_{i j}$ and compute its restriction to the boundary. The restriction of the corresponding $r_{i j}$ to the boundary will then represent the lift of $\left[\tilde{z}_{i}, \tilde{z}_{j}\right]$ back to $H^{1}\left(\partial X_{\gamma} ; \mathbb{R} i\right)$ from $H^{2}\left(X_{\gamma}, \partial X_{\gamma} ; \mathbb{R} i\right)$.

Since $H^{2}\left(X_{\gamma} ; \mathbb{R} i\right)=0$, there is some 1-cochain $s$ with boundary $\left[z_{i}, z_{j}\right]$. The 1-cocycles $Z^{1}\left(X_{y}, \mathbb{R} i\right)$ are defined by $x \mapsto X i, y \mapsto Y i$, $\tau \mapsto 0$ for any $X, Y \in \mathbb{R}$ so that $(a+1) X+c Y=0$. Notice that $c$ is nonzero, since $B$ has determinant 1 , and \pm 1 are not eigenvalues of $B$. Therefore, we can subtract a 1-cocycle $u$ from $s$ so that $(s-u)(x)=0$. Let $s_{i j}=s-u$. A long computation based on the identity

$$
s_{i j}(e)+e \cdot s_{i j}(f)-s_{i j}(e f)=\left[z_{i}(e), e \cdot z_{j}(f)\right]
$$

implies that $s_{i j}(y)$ is also equal to 0 .

Now (4) easily shows that

$$
s_{i j}(\mu)=\left[z_{i}(\tau),(1-y) z_{j}(\tau)\right] .
$$

Furthermore, $\left[z_{i}, v_{j}\right](\lambda)=\left[z_{i}, v_{j}\right](x)=0$ and

$$
\left[z_{i}, v_{j}\right](\mu)=\left[y^{d}(1-y) z_{i}(\tau), y^{d+1} \alpha z_{j}(\tau)\right] .
$$

This choice of $s_{i j}$ gives, via (3),

$$
r_{i j}(\lambda)=0
$$

and

$$
\begin{aligned}
r_{i j}(\mu)= & \left(s_{i j}+\left[z_{i}, v_{j}\right]\right)(\mu) \\
= & \left(1-y^{-1}+y^{-1}(1-y) \bar{\alpha}\right) z_{i}(\tau) z_{j}(\tau) \\
& -\left(1-y+y\left(1-y^{-1}\right) \alpha\right) z_{j}(\tau) z_{k}(\tau) .
\end{aligned}
$$

So, when $i \neq j$,

$$
r_{i j}(\mu)=2 \operatorname{Re}((y-1)(\alpha-1)) z_{i}(\tau) z_{j}(\tau),
$$

and when $i=j, z_{i}(\tau) z_{j}(\tau)-1$ and so

$$
\tau_{i j}(\mu)=2 \operatorname{Im}((y-1)(\alpha-1)) .
$$

One checks that

$$
(y-1)(\alpha-1)=-2 i \frac{\sin \left(\theta_{2}\right) \sin \left(d \theta_{2}\right)}{\sin \left((d+1) \theta_{2}\right)},
$$

so that (on the boundary) $r_{i j}=0$ if $i \neq j$, and $r_{11}(\mu)=r_{22}(\mu) \neq 0$, $r_{11}(\lambda)=r_{22}(\lambda)=0$. To finish the proof that the form is definite, we must 
project these to $H^{1}\left(\partial X_{y} ; \mathbb{R} i\right) / H^{1}\left(X_{y} ; \mathbb{R} i\right) \cong \mathbb{R}$. But $r_{11}$ and $r_{22}$ do not lie in the image of $H^{1}\left(X_{\gamma} ; \mathbb{R} i\right)$ in $H^{1}\left(\partial X_{\gamma} ; \mathbb{R} i\right)$. Lemma 7.7 states that a 1-cocycle $e$ on $\partial X_{\gamma}$ lies in this image if and only if $e(\mu)=b u+(d+1) v$ and $e(\lambda)=u$, for a pair $(u, v) \in \mathbb{R}^{2}$ satisfying $(a+1) u+c v=0$. Since $c \neq 0$ and $r_{i i}(\lambda)=0, r_{i i}(\mu) \neq 0$. The projection of $r_{i i}$ to $H^{1}\left(\partial X_{\gamma} ; \mathbb{R} i\right) / H^{1}\left(X_{\gamma} ; \mathbb{R} i\right)$ is nonzero. q.e.d.

We now present two applications of Theorem 5.1 to torus bundles. We continue to assume that $M$ is the $T^{2}$-bundle over $S^{1}$ with monodromy matrix $B \in S L_{2}(\mathbb{Z})$ given by

$$
B=\left(\begin{array}{ll}
a & b \\
c & d
\end{array}\right) .
$$

As before, we have $\Pi=\pi_{1} M$ presented by

$$
\Pi=\left\langle\tau, x, y \mid x y=y x, \tau x=x^{a} y^{b} \tau, \tau y=x^{c} y^{d} \tau\right\rangle .
$$

For $s \in \mathbb{R}$, define the abelian representation $\rho_{s}: \Pi \rightarrow s u(2)$ by

$$
\rho_{s}(\tau)=e^{i s}, \quad \rho_{s}(x)=\rho_{s}(y)=1 .
$$

The adjoint representation ad $\rho_{s}: \Pi \rightarrow \operatorname{Aut}(s u(2))$ preserves the splitting $s u(2)=\mathbb{R} i \oplus \mathbb{C} j$. Via ad $\rho_{s}, \Pi$ acts on $\mathbb{R} i$ trivially and on $\mathbb{C} j$ by

$$
\begin{gathered}
\operatorname{ad} \rho_{s}(x)=\operatorname{ad} \rho_{s}(y)=\mathrm{id}_{\mathbb{C} j}, \\
\operatorname{ad} \rho_{s}(\tau)(z j)=e^{2 i s} z j \quad \text { for } z \in \mathbb{C} .
\end{gathered}
$$

Let $H_{(s)}^{*}(M ; s u(2))$ denote the cohomology of $M$ with coefficients in $s u(2)$ twisted by the representation ad $\rho_{s}$. Cohomology computations may be made either using DeRham cohomology with an appropriate flat connection on $M \times s u(2)$ with (holonomy representation ad $\rho_{s}$ ), or using group cohomology as in the proof of Theorem 7.5. Both of these chain complexes respect the splitting $s u(2)=\mathbb{R} i \oplus \mathbb{C} j$. The coefficients in $\mathbb{R} i$ are trivial since the action of $\Pi$ on $\mathbb{R} i$ is trivial, so $H_{(s)}^{*}(M ; \mathbb{R} i) \cong H^{*}(M ; \mathbb{R})$. We begin by proving:

\section{Proposition 7.8.}

(1) $H_{(s)}^{0}(M ; \mathbb{R} i) \cong \mathbb{R} i$,

(2) $H_{(s)}^{1}(M ; \mathbb{R} i) \cong \mathbb{R} i \oplus \operatorname{ker}\left(B-I: \mathbb{R}^{2} \rightarrow \mathbb{R}^{2}\right)$,

$$
\begin{gathered}
H_{(s)}^{0}(M ; \mathbb{C} j) \cong \begin{cases}0 & \text { if } s \notin \mathbb{Z} \pi, \\
\mathbb{C} j & \text { if } s \in \mathbb{Z} \pi,\end{cases} \\
H_{(s)}^{1}(M ; \mathbb{C} j) \cong \begin{cases}\operatorname{ker}\left(B-e^{2 i s} I: \mathbb{C}^{2} \rightarrow \mathbb{C}^{2}\right) & \text { if } s \notin \mathbb{Z} \pi, \\
\mathbb{C} j \oplus \operatorname{ker}\left(B-I: \mathbb{C}^{2} \rightarrow \mathbb{C}^{2}\right) & \text { if } s \in \mathbb{Z} \pi .\end{cases}
\end{gathered}
$$


Proof. (1) is trivial.

To calculate $H^{1}(M ; \mathbb{R})$, we use group cohomology. A 1-cocycle $\alpha: \Pi \rightarrow$ $\mathbb{R}$ is determined by its values on the generators $\pi, x, y$. We need to see what conditions are imposed on these values by the relations for $\Pi$. First note that $x y=y x$ imposes no condition at all.

Next, applying the cocycle condition to $\alpha(\tau x)=\alpha\left(x^{\alpha} y^{b} \tau\right)$ and to $\alpha(\tau y)=\alpha\left(x^{c} y^{d} \tau\right)$ imposes the condition

$$
(B-I)\left(\begin{array}{c}
\alpha(x) \\
\alpha(y)
\end{array}\right)=0 .
$$

Since there is no condition on $\alpha(\tau)$ and there are no 1-coboundaries, we conclude (2) holds.

If $s \in \mathbb{Z} \pi$, the $\mathbb{C} j$ coefficients are also trivial (i.e., they are not twisted); it follows that $H_{(s)}^{0}(\Pi ; \mathbb{C} j) \cong \mathbb{C} j$ and

$$
H_{(s)}^{1}(\Pi ; \mathbb{C} j) \cong \mathbb{C} j \oplus \operatorname{ker}\left(B-I: \mathbb{C}^{2} \rightarrow \mathbb{C}^{2}\right) .
$$

Suppose that $s \notin \mathbb{Z} \pi$. Then for all $v \in \mathbb{C} j$

$$
\delta^{0} v(\tau)=\left(e^{2 i s}-1\right) v \neq 0
$$

so $H_{(s)}^{0}(\Pi ; \mathbb{C} j)=0$.

Suppose $\alpha: \Pi \rightarrow \mathbb{C} j$ is a 1-cocycle. Applying the cocycle condition to the group relations as above yields

$$
\left(B-e^{2 i s} I\right)\left(\begin{array}{l}
\alpha(x) \\
\alpha(y)
\end{array}\right)=0,
$$

as the only condition on $\alpha(x), \alpha(y)$, and $\alpha(\tau) \in \mathbb{C} j$. Hence

$$
Z_{(s)}^{1}(\Pi ; \mathbb{C} j) \cong \mathbb{C} \oplus \operatorname{ker}\left(B-e^{2 i s} I: \mathbb{C}^{2} \rightarrow \mathbb{C}^{2}\right),
$$

and since $\delta^{0}$ is injective as seen in the proof of (3), (4) follows. q.e.d.

In what follows, let $D_{s}=D_{A_{s}}$, where $A_{s}$ is a flat connection on $M \times s u(2)$ with holonomy $\rho_{s}$. We may assume that $D_{s}$ preserves the splitting $E=E_{i} \oplus E_{j}$.

Theorem 7.9. Assume $(B-I)$ is nonsingular. Then $\operatorname{ker} D_{0} \cong \mathbb{R}^{6}$. For $s \in(-\varepsilon, \varepsilon) \subset \mathbb{R}$, two of these eigenvalues are constant at 0 , two are crossing 0 with positive slope, and two are crossing 0 with negative slope.

Proof. By Proposition 7.8, $\operatorname{ker}\left(D_{s} \mid \Gamma\left(E_{i}\right)\right) \cong \mathbb{R}^{2}$ for all $s$; this gives our two eigenvalues which are constant at 0 .

Next, we calculate the bilinear form $G$ of Theorem 5.1 on $H^{0}(M ; s u(2))$ $\oplus H^{1}(M ; s u(2))$, where the coefficients are trivial since $s=0$. 
Let $e=1 \in \Omega^{0}(M ; \mathbb{R})$ and let $\alpha \in \Omega^{1}(M ; \mathbb{R})$ be a harmonic form normalized so that

$$
|\alpha|=\int_{M} \alpha \wedge * \alpha=1
$$

Let $v(M)=\int_{M} * e=$ volume of $M$. It follows that $H^{0}(M ; \mathbb{R})=\mathbb{R} e$, $H^{1}(M ; \mathbb{R})=\mathbb{R} \alpha, H^{2}(M ; \mathbb{R})=\mathbb{R}(* \alpha)$, and $H^{3}(M ; \mathbb{R})=\mathbb{R}(* e)$. In addition

$$
* e=v(M) \alpha \wedge * \alpha \quad \text { in } H^{3}(M ; \mathbb{R}) .
$$

Since our coefficients are trivial, we have

$$
H^{0}(M ; s u(2))=\mathbb{R} i e \oplus \mathbb{R} j e \oplus \mathbb{R} k e
$$

and

$$
H^{1}(M ; s u(2))=\mathbb{R} i \alpha \oplus \mathbb{R} j \alpha \oplus \mathbb{R} k \alpha .
$$

The tangent vector to our path of representations may be taken to be $\alpha=i \alpha$, so $* a=i(* \alpha)$. We then compute the matrix of $G$ to be:

$$
\begin{aligned}
& \text { ie } i \alpha \text { je ke } j \alpha \quad k \alpha
\end{aligned}
$$

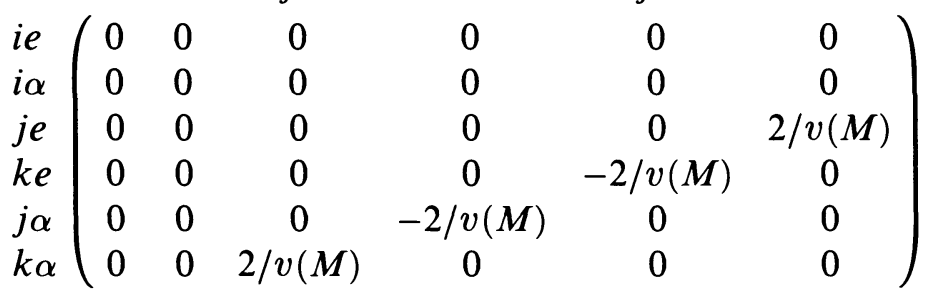

Here is a sample computation

$$
G(k \alpha, j e)=-[k \alpha, j e] \cdot i(* \alpha)=-[k, j] \cdot i(\alpha \wedge e \wedge * \alpha)=\frac{2}{v(M)}(* e) .
$$

The others are similar.

Because this matrix has rank 4 and is of the form

$$
\left(\begin{array}{ccc}
0 & 0 & 0 \\
0 & 0 & C \\
0 & C^{t r} & 0
\end{array}\right),
$$

where the blocks are $2 \times 2$, it follows that two eigenvalues are 0 , two are positive, and two are negative. Since we already know that the path $D_{s}$ has two eigenvalues which are constant at 0 , our current Theorem follows by applying Theorem 5.1 .

Remark. We may use Theorem 7.9 to calculate spectral flows from the trivial representation to certain noncentral abelian representations. Let $s_{0}>0$ in $\mathbb{R}$, and assume that for all $s$ with $0 \leq s \leq s_{0}, e^{2 i s}$ is 


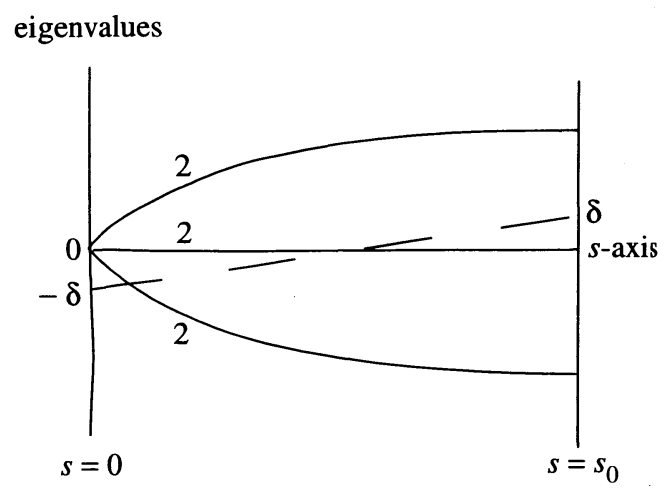

Figure 4

not an eigenvalue of $B$. Then Theorem 7.9 implies that $S F\left(\rho_{0}, \rho_{s_{0}}\right) \equiv$ $-4(\bmod 8)$. The situation is illustrated in Figure 4.

In Figure 4, we see four eigenvalues crossing the dotted line, all in the negative direction. The digit " 2 " next to a path of eigenvalues refers to the multiplicity of the eigenvalues in that path.

We now turn to a slightly more subtle application of Theorem 5.1. As before, assume that $M$ is the $T^{2}$-bundle over $S^{1}$ with monodromy matrix $B \in S L(2, \mathbb{Z})$.

Theorem 7.10. Suppose $B \in S L(2, \mathbb{Z})$ is a noncentral elliptic element, i.e., suppose $\operatorname{trace}(B) \in\{-1,0,1\}$. Assume $e^{2 i s_{0}}$ is an eigenvalue of $B$. Then

$$
H_{\left(s_{0}\right)}^{0}(M ; s u(2)) \cong \mathbb{R} \text { and } H_{\left(s_{0}\right)}^{1}(M ; s u(2)) \cong \mathbb{R}^{3}
$$

As $s$ passes through $s_{0}$, two eigenvalues stay constant at 0 , while the other two eigenvalues passing through 0 both have nonzero slope of the same sign. Hence $S F\left(\rho_{0}, \rho_{s_{0}+\varepsilon}\right) \equiv S F\left(\rho_{0}, \rho_{s_{0}-\varepsilon}\right) \pm 2(\bmod 8)$.

Remark. This result is not inconsistent with Jeffrey's conjecture [8, Conjecture 5.8]; see the footnote to Conjecture 5.8 and Remark 5.17 of [8].

Proof of Theorem 7.10. Let

$$
\left(\begin{array}{l}
z_{x} \\
z_{y}
\end{array}\right) \in \mathbb{C}^{2}-\left\{\left(\begin{array}{l}
0 \\
0
\end{array}\right)\right\}
$$

be an element of $\operatorname{ker}\left(B-e^{2 i s_{0}} I: \mathbb{C}^{2} \rightarrow \mathbb{C}^{2}\right)$. By the proof of Proposition 7.8, $H_{\left(s_{0}\right)}^{1}(\Pi ; \mathbb{C} j) \cong \mathbb{R} \alpha \oplus \mathbb{R} \beta$ where $\alpha$ and $\beta$ are 1-cocycles determined 
by

$$
\begin{aligned}
& \alpha(x)=z_{x} j, \quad \beta(x)=z_{x} k, \\
& \alpha(y)=z_{y} j, \quad \beta(y)=z_{y} k, \\
& \alpha(\tau)=0, \quad \beta(\tau)=0 .
\end{aligned}
$$

Also, $H_{\left(s_{0}\right)}^{0}(\Pi ; \mathbb{C} j)=0$ and $H_{\left(s_{0}\right)}^{1}(\Pi ; \mathbb{R} i)=\mathbb{R} \gamma$ where $\gamma(x)=\gamma(y)=0$ and $\gamma(\tau)=i$. Finally $H_{\left(s_{0}\right)}^{0}(\Pi ; \mathbb{R} i)=\mathbb{R} i$. Hence

$$
\operatorname{ker} D_{s_{0}} \cong \mathbb{R} i \oplus \mathbb{R} \gamma \oplus \mathbb{R} \alpha \oplus \mathbb{R} \beta
$$

We take $\gamma$ as the tangent vector to our path of flat connections at the point $\rho_{s_{0}}$. We now compute the bilinear form $G$ (on $\operatorname{ker} D_{s_{0}}$ ) defined in Theorem 5.1.

First, note that $[i, \gamma]=[i, i]=[\gamma, \gamma]=0$ because $[$,$] vanishes on$ $\mathbb{R} i$. Next, note that if $a \in \mathbb{R} i+\mathbb{R} \gamma$ and $b \in \mathbb{R} \alpha+\mathbb{R} \beta$, then $G(a, b)=0$ because $[a, b] \in \Omega^{*}(M ; \mathbb{C} j)$ while $\gamma$ and $* \gamma$ are in $\Omega^{*}(M ; \mathbb{R} i)$.

Hence we only need to compute $G(\alpha, \beta), G(\alpha, \alpha)$, and $G(\beta, \beta)$. We begin with $G(\alpha, \beta)$. We will show that $G(\alpha, \beta)=[\alpha, \beta] \cdot \gamma=0$ in $H^{3}(M ; \mathbb{R})$ by proving that $[\alpha, \beta]=0$ in $H_{\left(s_{0}\right)}^{2}(\Pi ; \mathbb{R} i)$. To accomplish this, we must construct a 1-cochain $r: \Pi \rightarrow \mathbb{R} i$ such that $\delta r=[\alpha, \beta]$. First define $r(\tau)=r(1)=0$. Then define $r(x), r(y) \in \mathbb{R} i$ to be the unique solution to the equation

$$
(B-I)\left(\begin{array}{l}
r(x) \\
r(y)
\end{array}\right)=\left(\begin{array}{l}
a(a-1) z_{x} \bar{z}_{x}+b(b-1) z_{y} \bar{z}_{y}+a b\left(z_{x} \bar{z}_{y}+\bar{z}_{x} z_{y}\right) \\
c(c-1) z_{x} \bar{z}_{x}+d(d-1) z_{y} \bar{z}_{y}+c d\left(z_{x} \bar{z}_{y}+\bar{z}_{x} z_{y}\right)
\end{array}\right) i
$$

Because our assumptions on $B$ imply that $B-I$ is nonsingular, this equation has a unique solution. By repeatedly applying the equation

$$
r(g h)=r(g)+g \cdot r(h)-[\alpha, \beta](g, h)
$$

(which is the meaning of the equation $\delta r=[\alpha, \beta]$ ) we find that $r$ satisfies the relations of $\Pi$, i.e.,

$$
r(x y)=r(y x), \quad r(\tau x)=r\left(x^{\alpha} y^{b} \tau\right), \quad r(\tau y)=r\left(x^{c} y^{d} \tau\right)
$$

It follows that $r$ extends (uniquely) to a 1-cochain on $\Pi$ satisfying $\delta r=[\alpha, \beta]$. Hence $[\alpha, \beta]=0=[\beta, \alpha]$ in $H_{\left(s_{0}\right)}^{2}(\Pi ; \mathbb{R} i)$.

We will now verify that $G(\alpha, \alpha)=G(\beta, \beta) \neq 0$. Since the map $H_{\left(s_{0}\right)}^{2}(M ; \mathbb{R} i) \rightarrow H^{3}(M ; \mathbb{R})$ defined by $u \mapsto u \cdot \gamma$ is an isomorphism (by Poincaré duality), it will suffice to show that $[\alpha, \alpha]=[\beta, \beta] \neq 0$ in $H_{\left(s_{0}\right)}^{2}(M ; \mathbb{R} i)$. That $[\alpha, \alpha]=[\beta, \beta]$ (as 2-cocycles!) follows from the facts that $\beta=i \alpha$ and ad $\rho_{s}$ preserves the complex structure on $\mathbb{C} j$. We 
now prove that $[\alpha, \alpha] \neq 0$ in $H_{\left(s_{0}\right)}^{2}(M ; \mathbb{R} i)$. Assume that $r: \Pi \rightarrow \mathbb{C}$ is a 1-cochain with $\delta r=[\alpha, \alpha]$. Then $r(x y)=r(y x)$ implies that

$$
r(x)+r(y)-[\alpha, \alpha](x, y)=r(y)+r(x)-[\alpha, \alpha](y, x) .
$$

Hence $[\alpha, \alpha](x, y)=[\alpha, \alpha](y, x)$, which shows that $[\alpha, \alpha](x, y)=$ $[\alpha(x), \alpha(y)]=0$. Substituting in $\alpha(x)=z_{x} j$ and $\alpha(y)=z_{y} j$ yields $-z_{x} \bar{z}_{y}+z_{y} \bar{z}_{x}=0$, i.e., $z_{y} \bar{z}_{x} \in \mathbb{R}$. We conclude after multiplying $\left(z_{x}, z_{y}\right)$ by either $\bar{z}_{x}$ or $\bar{z}_{y}$ that $B$ has a nonzero eigenvector in $\mathbb{R}^{2}$.

To see this is a contradiction, note that since $\operatorname{trace}(B) \in\{-1,0,1\}$, the only possible eigenvalues of $B$ are $\pm i, e^{ \pm \pi i / 3}, e^{ \pm 2 \pi i / 3}$. Since none of these are real (and since $B$ is real), $B$ cannot have an eigenvector in $\mathbb{R}^{2}$. This contradiction implies that $[\alpha, \alpha] \neq 0$ in $H^{2}(M ; \mathbb{R} i)$. It follows that the two nonzero eigenvalues of $G$ have the same sign and, by Theorem 5.1 , the two nonconstant eigenvalues of $D_{s}$ (which are passing through 0 at $s=s_{0}$ ) have the same nonzero slope. q.e.d.

\section{Loose ends}

There are several natural questions which are not resolved in this paper; here is a brief list.

1. If $Y$ is an arbitrary compact 3-manifold with boundary, then an appropriately adapted version of Theorem 6.4 can be formulated and proven for certain parameterized sets of flat connections on $\partial Y$. (Remark: this is proven in our recent article [11].)

2. Theorem 4.8 can be generalized in some way to cover what happens in the case where the centralizer of the holonomy of $\hat{A}_{t}$ jumps up in dimension at $t=0$.

3. If $M$ is a torus bundle over $S^{1}$ with $H^{1}(M ; \mathbb{R})=\mathbb{R}$, then corresponding to every pair of nonabelian representations of $\pi_{1}(M)$ in $S U(2)$ there is a "good pair" in $\mathbb{R}^{2}$ (in the sense defined in $\S 7$ ).

4. In a future article we will show that higher-order approximations to the spectral flow can be expressed in terms of higher-order cup products, i.e., Massey products.

\section{Appendix A. The analyticity of $D_{t}(r)$}

In this appendix we prove Theorem 4.6.

Let $A$ and $B$ be Banach spaces. Let $\mathscr{B}(A, B)$ denote the Banach space of bounded linear operators $A \rightarrow B$. Let $T(w) \in \mathscr{B}(A, B)$ for $w \in W$, a subdomain of $\mathbb{C}$. 
Definition. $\quad T(w)$ is bounded holomorphic on $W$ if for each fixed $a \in$ $A$ and $b \in B^{*}$ the function $\langle T(w) a, b\rangle$ is holomorphic at all $w \in W$.

This is not precisely the definition used in [9], but it is proven to be equivalent to his definition in [9, Theorem III.3.12, p. 152].

We now recall [9, p. 366] what it means for a family of closed but unbounded operators to be holomorphic. Let $T(w): A \rightarrow B$ be a family of closed operators, where the domain of $T(w)$ is $D(w) \subset A$, for $w \in W$, a subdomain of $\mathbb{C}$.

Definition. $T(w)$ is holomorphic (in the general sense) if there exist a Banach space $Z$ and a bounded holomorphic family $Q(w): Z \rightarrow A$ such that $Q(w)$ maps $Z$ bijectively onto $D(w)$ and such that $V(w)=$ $T(w) \circ Q(w)$ is a bounded holomorphic family.

It is in this general sense that we will show that $\mathscr{D}_{t}(r)$ is holomorphic in $t$.

First, we need to be very precise about domains. We define $\mathscr{D}_{i}(r)$ to be an operator in the Hilbert space $L^{2}(E(r))$ whose domain $D(t)$ is the image (under the natural inclusion) of $L_{1}^{2}\left(E(r) ; \mathscr{P}_{+}(t)+\mathscr{L}(t)\right)$ in $L^{2}(E(r))$.

Let $Z$ be the Banach space $L_{1}^{2}\left(E(r) ; \mathscr{P}_{+}(0)+\mathscr{L}(0)\right)$.

Let $\left\{\alpha_{k}(t)\right\}$ be a holomorphic family of orthonormal bases of $L^{2}(\widehat{E})$, and $\left\{\nu_{k}(t)\right\}$ a family of holomorphic functions such that for each $t$,

$$
\widehat{D}_{\widehat{A}_{t}} \alpha_{k}(t)=\nu_{k}(t) \alpha_{k}(t)
$$

Let $\left\{\beta_{1}(t), \cdots, \beta_{m}(t)\right\}$ be a holomorphic family of orthonormal bases for $\mathscr{L}(t)$. It follows that $\left\{\beta_{1}(t), \cdots, \beta_{m}(t), \sigma\left(\beta_{1}(t)\right), \cdots, \sigma\left(\beta_{m}(t)\right)\right\}$ is a holomorphic family of bases for $\mathscr{H}(t)$.

Define $\widetilde{U}_{t}: L^{2}(\widehat{E}) \rightarrow L^{2}(\widehat{E})$ as follows:

$$
\begin{aligned}
\tilde{U}_{t}\left(\alpha_{i}(0)\right) & =\alpha_{i}(t) \text { for } \nu_{i}(0) \neq 0, \\
\widetilde{U}_{t}\left(\beta_{i}(0)\right) & =\beta_{i}(t) \text { for } i=1, \cdots, m, \\
\widetilde{U}_{t}\left(\sigma\left(\beta_{i}(0)\right)\right) & =\sigma\left(\beta_{i}(t)\right) \text { for } i=1, \cdots, m .
\end{aligned}
$$

Because of our assumption that $\hat{A}_{t}$ is fine, it follows that $\tilde{U}_{t}$ is a bounded holomorphic family of operators on the Hilbert space $L_{1 / 2}^{2}(\widehat{E})$. Clearly, $\tilde{U}_{t}\left(\mathscr{P}_{+}(0)+\mathscr{L}(0)\right)=\mathscr{P}(t)+\mathscr{L}(t)$ for small values of $t$.

Define $R: L_{1}^{2}(E(r)) \rightarrow L_{1 / 2}^{2}(\widehat{E})$ to be the restriction of sections to the boundary. It is well-known (e.g., $[15$, p. 173]) that $R$ is a bounded surjective operator. It follows from the open mapping theorem that $R$ has 
a bounded right inverse $S: L_{1 / 2}^{2}(\widehat{E}) \rightarrow L_{1}^{2}(E(r))$. ( $S$ may be constructed using a $C^{\infty}$ cutoff function on $\Sigma \times[0, r]$.)

Now define a family of operators

$$
\widetilde{Q}_{t}: L_{1}^{2}\left(E(r) ; \mathscr{P}_{+}(0)+\mathscr{L}(0)\right) \rightarrow L_{1}^{2}(E(r))
$$

by $\widetilde{Q}_{t} g=g+S \circ\left(\widetilde{U}_{t}-\mathrm{Id}\right) \circ R(g)$. Since $\widetilde{U}_{t}$ is bounded holomorphic and $R$ and $S$ are bounded, it follows that $\widetilde{Q}_{t}$ is a bounded holomorphic family of operators. Furthermore, the image of $L_{1}^{2}\left(E(r) ; \mathscr{P}_{+}(0)+\mathscr{L}(0)\right)$ under $\widetilde{Q}_{t}$ is $L_{1}^{2}\left(E(r) ; \mathscr{P}_{+}(t)+\mathscr{L}(t)\right)$, and $\widetilde{Q}_{t}$ is a bijection onto this image. (In fact, one may easily write a formula for the inverse of $\widetilde{Q}_{t}$ on this image.)

Finally, let $Q_{t}=k \circ \widetilde{Q}_{t}$, where $k: L_{1}^{2}(E(r)) \rightarrow L^{2}(E(r))$ is the inclusion and is bounded (in fact compact). Then $Q_{t}(Z)=D(t)$, and $Q_{t}$ is the bounded holomorphic family of operators required by the definition of holomorphic family.

Finally, we need to see that $\mathscr{D}_{t}(r) \circ Q_{t}$ is a bounded holomorphic family. To this end, write

$$
\mathscr{D}_{t}(r) \circ Q_{t}=\left(\mathscr{D}_{t}(r) \circ k\right) \circ \widetilde{Q}_{t} .
$$

and note that each of these two operators are bounded holomorphic, since the first order part of $\mathscr{D}_{t}(r)$ is constant in $t$, and the zeroth order part is varying analytically. Hence we have shown that $\mathscr{D}_{t}(r)$ satisfies the definition of a holomorphic family in the general sense.

\section{Appendix B}

In this section we prove that analytic paths of connections on $T^{2}$ in "standard form" are fine, and prove Lemma 6.1. The idea which underlies both of the proofs is that the eigenspaces for the operators $\widehat{D}_{\widehat{A}}$ when $\widehat{A}$ is in standard form are independent of $\hat{A}$, though the eigenvalues are varying.

Throughout this section we use quaternionic notation for $s u(2)=\mathbb{R} i+$ $\mathbb{R} j+\mathbb{R} k=\mathbb{R} i+\mathbb{C} j$. We will write $T^{2}=\mathbb{R}^{2} / 2 \pi \mathbb{Z}^{2}$, and let $x$ and $y \in \mathbb{R}$ be the corresponding coordinates on $T^{2}$.

Our Riemannian metric on $T^{2}$ is inherited from the standard flat metric on $\mathbb{R}^{2}$. As a result, the Hodge star operates as follows:

$$
\begin{aligned}
*(1) & =d x d y, \\
*(d x) & =d y ; \quad *(d y)=-d x, \\
*(d x d y) & =1 .
\end{aligned}
$$

Of course $*$ acts as the identity on the coefficients. 
Definition. $w \in \Omega^{1}\left(T^{2} ; s u(2)\right)$ is in standard form if $w=$ aidx+ bidy where $a, b \in \mathbb{R}$ are constant on $T^{2}$. A connection $\widehat{A}$ on $T^{2} \times s u(2)$ is in standard form if $d_{\widehat{A}} u=d u+[w, u]$ for all $u \in \Omega^{*}\left(T^{2} ; s u(2)\right)$, where $w \in \Omega^{1}\left(T^{2} ; s u(2)\right)$ is in standard form.

Remark. In the above situation, we will often compress notation by writing $\widehat{A}=a i d x+b i d y$. If $\widehat{A}$ is in standard form, it follows that $\widehat{A}$ is flat with holonomy representation $\mu \mapsto e^{2 \pi i a}$ and $\lambda \mapsto e^{2 \pi i b}$ where $\mu$ and $\lambda$ are generators of $\pi_{1} T^{2}$ corresponding to the $x$ and $y$ coordinates. Note the trivial connection $\theta$ is in standard form, with $a=b=0$.

Theorem B.1. Let $\widehat{A}_{t}=a(t) i d x+b(t) i d y$ be a path of connections in standard form on $T^{2} \times S U(2)$, where $a$ and $b$ are analytic maps $(-\varepsilon, \varepsilon) \rightarrow \mathbb{R}$. For all $t \in(-\varepsilon, \varepsilon)$, assume that $(a(t), b(t)) \notin \frac{1}{2} \mathbb{Z}^{2}$. Then $\widehat{A}_{t}$ is a fine path of connections.

Proof. We may write the vector bundle $\widehat{E} \rightarrow T^{2}$ as a direct sum $\widehat{E}=$ $\widehat{E}_{i} \oplus \widehat{E}_{j}$ where

and

$$
\widehat{E}_{i}=\left(\Omega^{0} \oplus \Omega^{1} \oplus \Omega^{2}\right)\left(T^{2} ; \mathbb{R} i\right)
$$

$$
\widehat{E}_{j}=\left(\Omega^{0} \oplus \Omega^{1} \oplus \Omega^{2}\right)\left(T^{2} ; \mathbb{C} j\right) .
$$

It is easy to see that these direct summands are preserved by the operators $d_{\widehat{A}}, d_{\widehat{A}}^{*}, *, \Delta_{\widehat{A}}=d_{\widehat{A}} d_{\widehat{A}}^{*}+d_{\widehat{A}}^{*} d_{\widehat{A}}$ and $\widehat{D}_{\widehat{A}}$, if $\widehat{A}$ is a connection in standard form. Because Lie brackets vanish on $\mathbb{R} i$, it follows that if $\widehat{A}$ is in standard form, then $\widehat{D}_{\widehat{A}}=\widehat{D}_{\theta}$ and $d_{\widehat{A}}=d_{\theta}$ on $\Gamma\left(\widehat{E}_{i}\right)$. Thus if $\widehat{A}_{t}$ is a path of connections in standard form, we may take $U_{t} \mid \Gamma\left(\widehat{E}_{i}\right)=$ id for all $t$, which is certainly an analytic path of operators! Hence we only need to show that $U_{t} \mid \Gamma\left(\widehat{E}_{j}\right)$ is analytic. (The operator $U_{t}$ was introduced in $\S 4$, just before the definition of a fine path.)

Remark. A fact used repeatedly in the computations which follow is $[i a, z j]=2 a i \cdot z j$ where $a \in \mathbb{R}$ and $z \in \mathbb{C}$. As a result, note that $\Gamma\left(\hat{E}_{j}\right)$ has a complex structure which is preserved by all the operators we considering.

Define the function $\varphi_{m, n} \in \Omega^{0}\left(T^{2} ; \mathbb{C} j\right)$ by $\varphi_{m, n}(x, y)=e^{i m x+i n y} j$ for each $m, n \in \mathbb{Z}$. It is well-known that the $\left\{\varphi_{m, n}\right\}$ form an orthonormal basis for $L^{2}\left(\Omega^{0}\left(T^{2} ; \mathbb{C} j\right)\right)$ as a complex Hilbert space. For each $m, n$ define $V_{m, n} \subset \Gamma\left(\widehat{E}_{j}\right)$ by $V_{m, n}=$ complex $\operatorname{span}\left\{\varphi_{m, n}, \varphi_{m, n} d x, \varphi_{m, n} d y\right.$, $\left.\varphi_{m, n} d x d y\right\}$. It follows that as a complex (or real) Hilbert space, $\Gamma\left(\widehat{E}_{j}\right)=$ $\bigoplus_{m, n \in \mathbb{Z}} V_{m, n}$. It is easy to see that all of our operators preserve these direct summands if $\widehat{A}$ is in standard form. Another easy computation 
shows that if $\widehat{A}=a i d x+b i d y$, then

$$
\Delta_{\widehat{A}}\left(\varphi_{m, n}\right)=\mu_{m, n}(a, b) \varphi_{m, n},
$$

where $\mu_{m, n}(a, b)=(m+2 a)^{2}+(n+2 b)^{2}$. Since we assume that $(a, b) \notin$ $\frac{1}{2}(\mathbb{Z}+\mathbb{Z})$, it follows that $\operatorname{ker}\left(\Delta_{\vec{A}} \backslash \Omega^{0}\left(T^{2} ; \mathbb{C} j\right)\right)=0$. According to $\S 3$, we make the following definitions:

$$
\begin{aligned}
& \xi_{m, n}^{+}(a, b)=\left(\varphi_{m, n},-\frac{1}{\sqrt{\mu_{m, n}(a, b)}} * d_{\widehat{A}} \varphi_{m, n}, 0\right), \\
& \xi_{m, n}^{-}(a, b)=\left(\varphi_{m, n}, \frac{1}{\sqrt{\mu_{m, n}(a, b)}} * d_{\widehat{A}} \varphi_{m, n}, 0\right), \\
& \psi_{m, n}^{+}(a, b)=\left(0,-\frac{1}{\sqrt{\mu_{m, n}(a, b)}} d_{\widehat{A}} \varphi_{m, n}, * \varphi_{m, n}\right), \\
& \left.\psi_{m, n}^{-}(a, b)=\left(0, \frac{1}{\sqrt{\mu_{m, n}(a, b)}} d_{\widehat{A}} \varphi_{m, n}, * \varphi_{m, n}\right)\right)
\end{aligned}
$$

where $\widehat{A}=a i d x+b i d y$. By the material of $\S 3,\left\{\xi_{m, n}^{+}(a, b), \xi_{m, n}^{-}(a, b)\right.$, $\left.\psi_{m, n}^{+}(a, b), \psi_{m, n}^{-}(a, b)\right\}$ forms an orthogonal basis of $V_{m, n}$ as a complex Hilbert space. (Also, note that all of these basis elements have norm $=\sqrt{2}$.) To obtain a real basis we need to throw in the products of these elements with $i$. Furthermore, $\xi_{m, n}^{+}(a, b)$ and $\psi_{m, n}^{+}(a, b)$ are $\sqrt{\mu_{m, n}(a, b)}$-eigenvectors of $\widehat{D}_{\widehat{A}}$ and $\xi_{m, n}^{-}(a, b)$ and $\psi_{m, n}^{-}(a, b)$ are $-\sqrt{\mu_{m, n}(a, b)}$-eigenvectors of $\widehat{D}_{\widehat{A}}$.

Define $U_{t}^{m, n}: V_{m, n} \rightarrow V_{m, n}$ by

$$
U_{t}^{m, n}\left(\xi_{m, n}^{ \pm}(a(0), b(0))\right)=\psi_{m, n}^{ \pm}(a(t), b(t))
$$

and

$$
U_{t}^{m, n}\left(\psi_{m, n}^{ \pm}(a(0), b(0))\right)=\psi_{m, n}^{ \pm}(a(t) b(t)) .
$$

In what follows,we will write

$$
\begin{array}{ll}
a=a(0), & b=b(0), \\
\dot{a}=a^{\prime}(0), & \dot{b}=b^{\prime}(0) .
\end{array}
$$

Also, we will write $|u, v|=\sqrt{u^{2}+v^{2}}$. Define $\dot{U}^{m, n}=d U_{t}^{m, n} /\left.d t\right|_{t=0}$. 
We need to estimate $\left|\dot{U}^{m, n}\right|$. It will suffice to estimate the norm of $\left|\dot{U}^{m, n}\right|$ on each of the basis elements $\left\{\xi_{m, n}^{ \pm}(a, b), \psi_{m, n}^{ \pm}(a, b), i \xi_{m, n}^{ \pm}(a, b)\right.$, $\left.i \psi_{m, n}^{ \pm}(a, b)\right\}$. These computations are all similar; we will show how it goes for $\xi_{m, n}^{-}(a, b)$. First we compute

$$
U_{t}^{m, n}\left(\xi_{m, n}^{-}(a, b)\right)=\left(\varphi_{m, n}, \frac{1}{\sqrt{\mu_{m, n}(a(t), b(t))}} * d_{\widehat{A}_{t}} \varphi_{m, n}, 0\right) .
$$

We write

$$
B(t)=\frac{1}{\sqrt{\mu_{m, n}(a(t), b(t))}}=\frac{1}{\sqrt{(m+2 a(t))^{2}+(n+2 b(t))^{2}}}
$$

and

$$
C(t)=* d_{\widehat{A}_{t}} \varphi_{m, n}=-i(n+2 b(t)) \varphi_{m, n} d x+i(m+2 a(t)) \varphi_{m, n} d x .
$$

We then estimate:

$$
\begin{aligned}
|B(0)| & =\frac{1}{|m+2 a, n+2 b|}, & & \left|B^{\prime}(0)\right| \leq \frac{2|(\dot{a}, \dot{b})|}{|m+2 a, n+2 b|^{2}}, \\
|C(0)| & =|m+2 a, n+2 b|, & & \left|C^{\prime}(0)\right| \leq 2|\dot{a}, \dot{b}| .
\end{aligned}
$$

Hence

$$
\left|\dot{U}^{m, n}\left(\xi_{m, n}^{-}(a, b)\right)\right|=\left|B^{\prime}(0) C(0)+B(0) C^{\prime}(0)\right| \leq \frac{4|\dot{a}, \dot{b}|}{|m+2 a, n+2 b|} .
$$

A similar computation for the other seven basis elements gives the same result. Hence, we obtain

$$
\left|\dot{U}^{m, n}\right| \leq \frac{32}{\sqrt{2}} \frac{|\dot{a}, \dot{b}|}{|m+2 a, n+2 b|} .
$$

Clearly, $\dot{U}=\bigoplus_{m, n \in \mathbb{Z}^{2}} \dot{U}^{m, n}$, an orthogonal direct sum. Note that $\lim _{|m, n| \rightarrow \infty}\left|\dot{U}^{m, n}\right|=0$. Hence $|\dot{U}|<\infty$, and $\dot{U}$ is a bounded operator. The same argument yields that $U_{t}$ is differentiable at all small real values of $t$. To conclude that $U_{t}$ is a bounded holomorphic family, we need to show that $U_{t}$ is differentiable for small complex values of $t$. To make sense of this, first note that

$$
\Omega^{*}\left(T^{2} ; s u(2)\right) \otimes_{R} \mathbb{C}=\Omega^{*}\left(T^{2} ; s l_{2} \mathbb{C}\right) .
$$


Hence using the same power series for $\widehat{A}_{t}$ but allowing complex values for $t$ gives us a 1-(complex parameter) family of 1 -forms in $\Omega^{1}\left(T^{2} ; s l_{2} \mathbb{C}\right)$. With this modification the same formulas for all of the operators, eigenvectors, and eigenvalues continue to hold, and so do the estimates. As a result we see that $U_{t}$ is differentiable for small values of $t \in \mathbb{C}$.

To complete the proof that $\widehat{A}_{t}$ is a fine arc of connections, we need to prove that $\dot{U}$ is also a bounded linear function in the $L_{1 / 2}^{2}$-norm of $\Gamma(\widehat{E})$. To see this note the following facts:

(1) The spaces $V_{m, n}$ are still orthogonal with respect to the $L_{1 / 2}^{2}$-inner product.

(2) On each $V_{m, n}$, the $L_{1 / 2}^{2}$-norm is a constant multiple (the constant is $\left.\left(1+m^{2}+n^{2}\right)^{1 / 4}\right)$ of the $L^{2}$-norm.

Hence the $L_{1 / 2}^{2}$-norm of each $\dot{U}^{m, n}$ is the same as our $L^{2}$-norm. It follows that $\dot{U}$ is bounded on $L_{1 / 2}^{2}(\widehat{E})$. This shows that $U_{t}$ is a bounded holomorphic family of operators on $L_{1 / 2}^{2}$, and completes the proof that $\widehat{A}_{t}$ is a fine family of connections on $T^{2}$.

We now turn to the proof of Lemma 6.1.

Proof of Lemma 6.1. We use the coordinates in $\mathbb{R}^{2}$ to label points in $R$. We will show that the map taking $((a, b), L)$ to $\mathscr{D}_{a, b}\left(P_{a, b}(+)+L\right)$ is continuous.

Let $\mathscr{O} \Subset R$ be an open disc and let $r>0$ so that $\operatorname{dist}\left(\mathscr{O},\left(\frac{1}{2} \mathbb{Z}\right)^{2}\right)>r$. (The metric in $R$ is taken to be the usual Euclidean metric in $R \subset \mathbb{R}^{2}$.)

We will measure the distance between Lagrangians in the following way: Fix a Lagrangian $L_{0}$. The orbit map $\operatorname{Sp}(\mathscr{H}) \rightarrow \operatorname{Lag}(\mathscr{H})$ sending $g$ to $g \cdot L_{0}$ is a fiber bundle. Let $\mathscr{N}$ be a small geodesic ball centered at $L_{0}$ in $\operatorname{Lag}(\mathscr{H})$. Choose a (continuous) local section $f: \mathscr{N} \rightarrow \operatorname{Sp}(\mathscr{H})$ sending $L_{0}$ to the identity. Then define the distance from $K$ to $L$ in $\mathscr{N}$ to be the distance from $f(K)$ to $f(L)$ in $\operatorname{Sp}(\mathscr{H}) \subset \mathbb{R}^{16}$. We use the product metric on $\mathscr{O} \times \mathscr{N} \subset \mathrm{Lag}$.

Suppose that $((a, b), L) \in \mathscr{O} \times \mathscr{N}$, and let $\left(\left(a_{k}, b_{k}, L_{k}\right) \in \mathscr{O} \times \mathscr{N}\right.$ so that $\left(\left(a_{k}, b_{k}\right), L_{k}\right)$ converges to $((a, b), L)$ as $k \rightarrow \infty$. Notice that $f\left(L_{k}\right) f(L)^{-1} \in \operatorname{Sp}(\mathscr{H})$ takes $L$ to $L_{k}$.

Split the bundle $\widehat{E}=\widehat{E}_{i} \oplus \widehat{E}_{j}$ as before. For each $k$, define the isometry $U_{k}: L^{2}(\widehat{E}) \rightarrow L^{2}(\widehat{E})$ as follows:

1. On $\mathscr{H} \subset L^{2}\left(\widehat{E}_{i}\right)$, let $U_{k}=f\left(L_{k}\right) f(L)^{-1}$.

2. On $\mathscr{H}^{\perp} \cap L^{2}\left(\widehat{E}_{i}\right)$, let $U_{k}=\mathrm{id}$. 
3. On $L^{2}\left(\widehat{E}_{j}\right)$, let $U_{k}$ take $\xi_{m, n}^{ \pm}(a, b) \tau$ to $\xi_{m, n}^{ \pm}\left(a_{k}, b_{k}\right) \tau$, and $\psi_{m, n}^{ \pm}(a, b) \tau$ to $\psi_{m, n}^{ \pm}\left(a_{k}, b_{k}\right) \tau$, for $\tau=1$ or $i$.

We will need an extension of this construction. For each $k$, consider the straight line path $\left(a_{k}(t), b_{k}(t)\right)=(a, b)+t\left(a_{k}-a, b_{k}-b\right)$ in $\mathscr{O}$. Let $L_{k}(t)$ be a path of Lagrangians in $\mathscr{N}$ from $L$ to $L_{k}$. For definiteness one can choose the geodesic from $L$ to $L_{k}$. Then for each $k$ define a path $U_{k}(t)$ of unitary transformations in the same way that $U_{k}$ was defined. So $U_{k}(0)=$ id and $U_{k}(1)=U_{k}$.

Notice that $\Gamma\left(\widehat{E}_{i}\right)$ is pointwise orthogonal to $\Gamma\left(\widehat{E}_{j}\right)$, so that we have $L_{s}^{2}(\widehat{E})=L_{s}^{2}\left(\widehat{E}_{i}\right) \oplus L_{s}^{2}\left(\widehat{E}_{j}\right)$ for $s=0, \frac{1}{2}$. Thus

$$
\begin{aligned}
\left\|U_{k}-\mathrm{id}\right\|_{L_{1 / 2}^{2} \leq} \leq & \left\|\left(U_{k}-\mathrm{id}\right)_{\mid \mathscr{H}}\right\|_{L_{1 / 2}^{2}} \\
& +\left\|\left(U_{k}-\mathrm{id}\right)_{\mid \mathscr{H}^{\perp} \cap L_{1 / 2}^{2}\left(\widehat{E}_{i}\right)}\right\|_{L_{1 / 2}^{2}}+\left\|\left(U_{k}-\mathrm{id}\right)_{\mid L_{1 / 2}^{2}\left(\widehat{E}_{j}\right)}\right\|_{L_{1 / 2}^{2}} .
\end{aligned}
$$

The first term is bounded by $K_{1} \operatorname{dist}\left(L, L_{k}\right)$ for some $K_{1}$ depending only $f: \mathscr{N} \rightarrow \operatorname{Sp}(\mathscr{H})$. The second term is zero. We need to bound the third term.

Split $\Gamma\left(\widehat{E}_{j}\right)$ into the sum of the $V_{m, n}$ 's as in the previous theorem. We will bound $\dot{U}_{k}=d U_{k} / d t$ on $\Gamma\left(\widehat{E}_{j}\right)$ and then integrate from 0 to 1 to bound $\left\|U_{k}-\mathbf{I d}\right\|_{E_{j}}$.

Let $B(t), C(t)$ be defined as before. Then

$$
\left\|\dot{U}_{k}^{m, n} \xi_{m, n}^{-}(a, b)\right\| \leq\left|B^{\prime}(t)\right|\|C(t)\|+|B(t)|\left\|C^{\prime}(t)\right\| .
$$

As before, we have:

$$
\begin{array}{ll}
|B(t)|=\frac{1}{\left|m+2 a_{k}(t), n+2 b_{k}(t)\right|}, & \|C(t)\| \leq\left|m+2 a_{k}(t), n+2 b_{k}(t)\right|, \\
\left|B^{\prime}(t)\right| \leq \frac{\left|a_{k}-a, b_{k}-b\right|}{\left|m+2 a_{k}(t), n+2 b_{k}(t)\right|^{2}}, & \left\|C^{\prime}(t)\right\|=\frac{2 \sqrt{2}\left|a_{k}-a, b_{k}-b\right|}{\left|m+2 a_{k}(t), n+2 b_{k}(t)\right|} .
\end{array}
$$

Since $\left(a_{k}(t), b_{k}(t)\right)$ lies entirely in the $\operatorname{disc} \mathscr{O}$,

$$
\left|m+2 a_{k}(t), n+2 b_{k}(t)\right|>2 r .
$$

Hence

$$
\left\|\dot{U}^{m, n} \xi_{m, n}^{-}(a, b)\right\| \leq \frac{2 \sqrt{2}+1}{2 r}\left|a_{k}-a, b_{k}-b\right| .
$$

Similar estimates apply to the other basis elements of $V_{m, n}$ and so there is a constant $K_{2}$ depending only on $\mathscr{O}$ (and in particular independent of $m, n)$ so that

$$
\left\|\dot{U}_{k \mid V_{m, n}}\right\| \leq K_{2} \operatorname{dist}\left((a, b),\left(a_{k}, b_{k}\right)\right)
$$


This can be taken in $L^{2}$ or $L_{1 / 2}^{2}$ by the remark preceding the proof of this lemma. Since $\dot{U}_{k}$ preserves the $V_{m, n}$, we conclude that

$$
\left\|\dot{U}_{k}\right\|_{L_{1 / 2}^{2}\left(\widehat{E}_{j}\right)} \leq K_{2} \operatorname{dist}\left((a, b),\left(a_{k}, b_{k}\right)\right)
$$

and so

$$
\left\|\left(U_{k}-\mathrm{id}\right)_{\mid \widehat{E}_{j}}\right\| \leq \int_{0}^{1}\left\|\dot{U}_{k}(t)\right\| \leq K_{2} \operatorname{dist}\left((a, b),\left(a_{k}, b_{k}\right)\right)
$$

Now let $R: L_{1}^{2}(E) \rightarrow L_{1 / 2}^{2}(\widehat{E})$ be the restriction to the boundary, and $S: L_{1 / 2}^{2}(\widehat{E}) \rightarrow L_{1}^{2}(E)$ a bounded right inverse, and as in appendix A define the bounded operator $\widetilde{Q}_{k}: L_{1}^{2}\left(E ; P_{a, b}(+)+L\right) \rightarrow L_{1}^{2}\left(E ; P_{a_{k}, b_{k}}(+)+L_{k}\right)$ by $\widetilde{Q}_{k}(g)=g+S(U(R(g))-R(g))$.

Let $Q_{k}: L_{1}^{2}\left(E ; p P_{a, b}(+)+L\right) \rightarrow L^{2}(E)$ be the composite of $\widetilde{Q}_{k}$ and the inclusion $i$ of $L_{1}^{2}(E)$ into $L^{2}(E)$. Write $\mathscr{D}$ for $\mathscr{D}_{a, b}(L)$ and $\mathscr{D}_{k}$ for $\mathscr{D}_{a_{k}, b_{k}}\left(L_{k}\right)$. So the image of $Q_{k}$ is the domain of $\mathscr{D}_{k}$, and $Q_{k}$ is bounded; moreover:

$$
\begin{aligned}
\left\|Q_{k}-\mathrm{id}\right\| & \leq\|i\|\|S\|\left\|U_{k}-\mathrm{id}\right\|_{L_{1 / 2}^{2}\|R\|} \\
& \leq K_{3}\left(\operatorname{dist}\left((a, b),\left(a_{k}, b_{k}\right)\right)+\operatorname{dist}\left(L, L_{k}\right)\right)
\end{aligned}
$$

for some $K_{3}$ depending only on $r$ and $f$. Thus $\lim _{k}\left\|Q_{k}-\mathrm{id}\right\|=0$.

Now let $V_{k}=\mathscr{D}_{k} \circ Q_{k}$. If $x \in L_{1}^{2}\left(E ; P_{a, b}(+)+L\right)$, then

$$
\begin{aligned}
\left\|\left(V_{k}-\mathscr{D}\right) x\right\|_{L^{2}} & \leq\left\|\mathscr{D}_{k} Q_{k} x-\mathscr{D}_{k} x\right\|_{L^{2}}+\left\|\mathscr{D}_{k} x-\mathscr{D} x\right\|_{L^{2}} \\
& \leq\left(\left\|\mathscr{D}_{k}\right\|\left\|Q_{k}-\mathrm{Id}\right\|+\left\|\mathscr{D}_{k}-\mathscr{D}\right\|\right)\|x\|_{L_{1}^{2}}
\end{aligned}
$$

where $\left\|\mathscr{D}_{k}\right\|$ means the norm of $\mathscr{D}$ viewed as an operator from all of $L_{1}^{2}$ to $L^{2}$ and similarly $\left\|\mathscr{D}_{k}-\mathscr{D}\right\|$. But the operators $D_{A}: L_{1}^{2}(E) \rightarrow L^{2}(E)$ all have the same first order part, and their difference is a zeroth order operator. In particular $\left\|D_{A}-D_{B}\right\|$ varies continuously in $A$ and $B$. Thus $\left\|\mathscr{D}_{k}\right\|$ is bounded, and $\left\|\mathscr{D}_{k}-\mathscr{D}\right\|$ goes to zero as $k \rightarrow \infty$. Hence $\lim _{k}\left\|V_{k}-\mathscr{D}\right\|=0$.

Now Lemma IV.2.29 of [9] implies that $\mathscr{D}_{k} \rightarrow \mathscr{D}$ in the graph topology, so that $\mathscr{D}_{A}\left(L_{A}\right)$ defines a continuous map from Lag to the space of closed, selfadjoint operators. 


\section{References}

[1] M. Atiyah, V. Patodi \& I. Singer, Spectral asymmetry and Riemannian geometry. I, II, III, Math Proc. Cambridge Philos. Soc. 77, 78, 79 (1975) 43-69; 405-432; 71-99.

[2] D. Auckly, Computing secondary and spectral invariants, Thesis, Univ. of Michigan, 1991.

[3] K. Brown, Cohomology of groups, Graduate Texts in Math., Springer, Berlin.

[4] S. Cappell, R. Lee \& E. Miller, Self-adjoint elliptic operators and manifold decomposition. I: Low eigenmodes and stretching, preprint, 1992.

[5] __ Self-adjoint elliptic operators and manifold decomposition. II, preprint 1992.

[6] R. Fintushel \& R. Stern, Instanton homology of Seifert-fibered homology spheres, Proc. Lond. Math. Soc. (3) 61 (1990) 109-137.

[7] A. Floer, Instanton invariant for 3-manifolds, Comm. Math. Phys. 118 (1989) 215-240.

[8] L. Jeffrey, Chern-Simons-Witten invariants of lens spaces and torus bundles and the semi-classical approximation, Comm. Math. Phys. 147 (1992) 563-604.

[9] T. Kato, Perturbation theory for linear operators, 2nd ed., Grundlehren Math. Wiss. No. 132, Springer, Berlin, 1976.

[10] P. Kirk \& E. Klassen, Chern-Simons invariants of 3-manifolds and representation spaces of knot groups, Math. Ann. 287 (1990) 347-367.

[11] _ , The spectral flow of the odd signature operator on a manifold with boundary, preprint, 1993.

[12] E. Klassen, Representation spaces of knot groups in $S U(2)$, Trans Amer. Math. Soc. 326 (1991) 795-828.

[13] T. Mrowka \& K. Walker, preprint, 1993.

[14] L. Nicolaescu, The Maslov index, the spectral flow, and splittings of manifolds, 1993.

[15] R. Palais, Seminar on the Atiyah-Singer index theorem, Annals. of Math. Studies, No. 57, Princeton Univ. Press, Princeton, NJ, 1965.

[16] C. Taubes, Gauge theory and Casson's invariant, J. Differential Geometry 31 (1990) 547-599.

[17] F. Warner, Foundations of differentiable manifolds and Lie groups, Graduate Texts in Math., No. 94, Springer, Berlin, 1983.

[18] E. Witten, Quantum field theory and the jones polynomial, Comm. Math. Phys. 121 (1989) 351-399.

[19] T. Yoshida, Floer homology and splittings of manifolds, Ann. Math. (2) 134 (1991) 277-324.

[20] _ A A computational algorithm of spectral flow for Floer homology, Topology '90, Walter de Gruyter, Berlin, 1992. 